\title{
Transform Domain Digital Watermarking with Multiple Images as Watermarks
}

\author{
${ }^{1}$ Amita Singha, ${ }^{2}$ Dr. Muhammad Ahsan Ullah \\ ${ }^{1} \mathrm{PhD}$ Student, Department of Electrical and Electronic Engineering \\ Chittagong University of Engineering and Technology \\ Chittagong-4349, Bangladesh \\ Email: amitasingha@yahoo.com \\ ${ }^{2}$ Professor \& Head, Department of Electronics \& Telecommunication Engineering, \\ Chittagong University of Engineering and Technology \\ Chittagong-4349, Bangladesh \\ Email: ahsan_cuet@yahoo.com
}

\begin{abstract}
In this paper, a technique of image watermarking using multiple images as watermarks is presented. The technique is based on transform domain functions including discrete wavelet transform (DWT), discrete cosine transform (DCT) and singular value decomposition (SVD) with an image as the host signal i.e. the watermarks will be used as proofs of the authenticity of the host image. The technique is executed by performing multilevel DWT followed by applying DCT and SVD to both the host and watermark. Multiple watermarks are used for the insurance of better security level. The scheme is immune to common image processing operations \& some attacks and exhibits PSNR of $108.3781 \mathrm{~dB}$, normalized cross correlation (NCC) over 0.99 and normalized correlation (NC) over 0.99 .
\end{abstract}

Keywords: Digital Watermarking; Multiple Image; Transform Domain.

\subsection{Image Watermarking}

Image watermarking is defined as the process of embedding information in an image which will act as the host image. The embedded information can be any type of media like audio, text or another image. The embedded information should be protected and extracted which will be used for copyright protection and the verification of the host image. Digital image watermarking can be of two types: visible and invisible. The requirements of invisible watermarking are imperceptibility. On the other hand, in the case of visible watermarking, the watermark should be visible so that the hidden data can be easily detected [2]. Due to the rapid increase of sharing of data through the internet because of the higher speed of information transmission and reception, 
it has become very important to protect the authenticity of a signal being sent. It is also very important to provide security to multimedia as well as to protect it from various forms of forgery in an efficient manner with respect to the speed of verification process [3]. Image watermarking can be done on the spatial domain as well as on the transform domain. Several functions were used to implement the transform domain watermarking i.e. the watermarking algorithm can be developed by using transform domain functions such as discrete cosine transform(DCT) [4-5], discrete wavelet transform (DWT) [6-7], singular value decomposition (SVD) [8-10], fast Fourier transform (FFT), contour-let transform (CT), and Wigner transform (WT), etc. Before watermarking, some modifications can be done on the host image to increase the efficiency of watermarking [11]. A modification can be also done on the watermark which is proved to be a very powerful factor for better performance [12]. The combination of different transform domain functions is used to develop very powerful watermarking algorithm [13-14]. Further, the use of multiple watermarks can add an extra dimension to the achievement of privacy and authenticity [15-16]. Another method of multiple-watermarking is inserting the same information several times into the host image. This method is very fruitful in increasing the visual and statistical invisibility of hidden information [17].

\subsection{Image Watermarking with Image as the Watermarks}

The proposed technique is based on transform domain functions including discrete wavelet transform (DWT), discrete cosine transform (DCT) and singular value decomposition (SVD). The watermarking is executed by performing multilevel DWT followed by applying DCT and SVD to both the host and watermark images. The use of multiple watermarks is to ensure better security level. The experimental results demonstrate that the proposed scheme is immune to common image processing operations and some attacks such as salt \& pepper noise, Gaussian noise, rotation, filtering, compression, etc. The technique proposed, exhibits PSNR of 108.3781dB, normalized cross-correlation (NCC) over 0.99 and normalized correlation (NC) over 0.99 i.e. this technique can extract the watermarks efficiently and also ensures good robustness. This technique is also tested for one and two watermarks. The values of PSNR for one and two watermarks are $108.3781 \mathrm{~dB}$ and $108.3781 \mathrm{~dB}$ respectively.

\subsubsection{Explanation of the Proposed Technique}


In many of the papers on 'Digital Watermarking', the proposed techniques incorporate the embedding technique using a scaling factor. For extraction, the scaling factor remains the same. Only one watermark is used and at best 3-level DWT is used. But, in the proposed technique, multi-level DWT along with multiple signals as watermarks were used. In the proposed technique, 4-level DWT was used for decomposing the host signals and 2-level DWT for decomposing watermark signals. $5^{\text {th }}, 6^{\text {th }}$, and $7^{\text {th }}$ level DWT were obtained by decomposing three sub-bands obtained by the 4th level of decomposition of host signals. Also, in both the techniques, three images were used as watermarks to increase the security level. The same value of coefficient was not used to combine the host and watermark images for embedding and extraction of watermarks so that information content of watermark images in the host signals changes during embedding and extraction of the watermarks without changing the perceptual content of watermark images. This assumption proved to be very effective which can be seen by observing the values of PSNR, NC and NCC. The watermarks were embedded in three different sub-bands of host signals. The size of both host images and watermark images changed prior to decomposition for better performance. DCT was used in every sub-band achieved after every level of decomposition using DWT although SVD was used in selected sub-bands. The technique was also used for one and two watermarks to compare the imperceptibility as more watermarks means more noise that can degrade the quality of the host image. But from the values of PSNR, it is evident that, the difference in the values of PSNR is too small to be considered. So, it can be said that, the proposed technique can be helpful both for single watermark as well as multiple watermarks. Also, for one and two watermarks, the levels of decomposition using DWT will be decreased by two levels and one level respectively. Also, multilevel DWT and the algorithm used for combining host and watermark images made the technique immune to the noisy effect created by using images as watermarks.

\subsubsection{Embedding Technique}

In the proposed technique, DWT transforms an image into four non-overlapping bands: LL, HL, LH, and HH. The bands represent approximate, horizontal, vertical and diagonal sub-bands, respectively. The theory behind the proposed method is: decomposing both the host image and the watermark images into four non-overlapping sub-bands. LL sub-band of host image will be combined with the HL sub-band of watermark image because maximum image energy is 
concentrated into the LL sub-band. So, this combination will not distort the quality of host image and the watermark will be invisible. Also, the extraction of the watermark will be up to the mark though some changes will occur on the horizontal components of the watermark as the human eye is less sensitive to changes in horizontal components. Next, the LH sub-band of the host image will be combined with the $\mathrm{HH}$ sub-band of the watermark image. In this case, also the invisibility will be maintained. It is known thatthe human eye can sense the changes in vertical components more easily. So, by combining the $\mathrm{HH}$ sub-band of the watermark image with the LH sub-band of the host image should change the LH sub-band of the host image. But, this will not happen as the HH sub-band contains very less information about the watermark image. So, the maintenance of the invisibility of the watermarks can be ensured. The next two combinations will be HL sub-band of the host image with the LL sub-band of the watermark image and $\mathrm{HH}$ sub-band of the host image with the LH sub-band of the watermark image. After these combinations, four sub-bands of the watermarked image will be obtained. Although, the HL subband of the watermarked image contains the LL sub-band of watermark image where maximum image energy is concentrated, the HL sub-band of the watermarked image itself contains less image energy as all the combinations will take place by using only a small fraction of sub-bands of the watermark image so this combination will not cause the visibility of watermark image which will be seen later. In the proposed methodology both the host and the watermark images are decomposed using DWT several times. The reason behind this proposal is: if the images are decomposed one time and combined, then the extraction of the watermark image and thus to change it will be comparatively easy as the number of permutations \& combinations needed to decode the embedding algorithm is poor. If the images are decomposed 2 times, the possible number of permutations \& combinations will be increased but the possibility of changing the watermark will not be low enough to ensure better performance. For a further level of decomposition, the difficulty level goes on increasing as the possible number of permutations $\&$ combinations goes on increasing in every level and the extraction of the watermark will be very difficult. In the proposed techniques, both the host image and the watermark images are decomposed rather than decomposing only the host images which added an extra advantage. A comparatively large value of PSNR (Peak Signal to Noise Ratio) can be achieved which is a very important factor of 'Digital Watermarking', which will be seen later. The proposed techniques 
used three watermarks. The reason is, it is comparatively easy to extract one watermark rather than three watermarks.

In the technique, DCT was applied in every sub-band of every level of DWT of host image and watermark images and singular values of desired sub-bands of host and watermark images are modified. Human eyes are less sensitive to small alteration of singular values. So, applying SVD along with DWT and DCT yields a good result for 'Digital Watermarking'. In the second technique, SVD along with multilevel DWT was used.

In the technique, very high values of PSNR and NC and NCC over 0.99 were achieved. This is a very good combination. A very high value of PSNR means that the watermarks imposed very less effect on the host image. But, if a very less effect of watermarks is used, then the quality of the extracted watermarks should be poor. But, that was not which is evident from the values of $\mathrm{NC}$ and NCC as NC and NCC are the measures of similarity between the watermark images before and after being extracted. The reason is that the size of the host image is much larger than the size of watermarks. In a nutshell, the proposed techniques are based on inserting very small watermarks into a comparatively large host image so that the watermarks can have very less distortion effect on the host image as well as the maintenance of the authenticity of the watermarks after extraction.

\subsubsection{Embedding Procedure}

\section{Host Image:}

1. Load a picture (Standard Lena Image) of size $512 \times 512$. Increase its size by 4 .

2. Apply $1^{\text {st }}$ level DWT (Discrete Wavelet Transform) on the image and decompose it into LL1, LH1, HL1, and HH1 sub-bands.

3. Apply $2^{\text {nd }}, 3^{\text {rd }}$ and $4^{\text {th }}$ level DWT on HH sub-bands which are got from $1^{\text {st }}, 2^{\text {nd }}$ and $3^{\text {rd }}$ level of decomposition respectively and get LL2, LH2, HL2 \& HH2 sub-bands, LL3, LH3, HL3 \& HH3 sub-bands and LL4, LH4, HL4 \& HH4 sub-bands from $2^{\text {nd }}, 3^{\text {rd }}$ and $4^{\text {th }}$ level DWT respectively.

4. $5^{\text {th }}$ level decomposition will be got by decomposing the LH sub-band to another 4 sub-bands which was got by the $4^{\text {th }}$ level of decomposition of the host image and get LL5, LH5, HL5, and HH5 sub-bands. 
5. $6^{\text {th }}$ level decomposition will be got by decomposing the HL sub-band to another 4 sub-bands which was got by the $4^{\text {th }}$ level of decomposition of the host image and get LL6, LH6, HL6, and HH6 sub-bands.

6. $7^{\text {th }}$ level decomposition will be got by decomposing the HH sub-band to another 4 sub-bands which was got by the $4^{\text {th }}$ level of decomposition of the host image and get LL7, LH7, HL7, and HH7 sub-bands.

7. Then apply DCT (Discrete Cosine Transform) to every sub-band achieved by every level of decomposition using DWT.

8. Further apply SVD (Singular Value Decomposition) to the sub-bands achieved by $5^{\text {th }}, 6^{\text {th }}$ and $7^{\text {th }}$ level of decomposition using DWT.

\section{Watermark Images:}

1. Load three pictures of size $512 \times 512$. Decrease its size by 2 .

2. Apply $1^{\text {st }}$ level DWT (Discrete Wavelet Transform) on the images and decompose it into LLW1, LHW1, HLW1, and HHW1 sub-bands.

3. Apply $2^{\text {nd }}$ level DWT on HH sub-bands which are got from $1^{\text {st }}$ level of decomposition and get LLW2, LHW2, HLW2, and HHW2 sub-bands. 


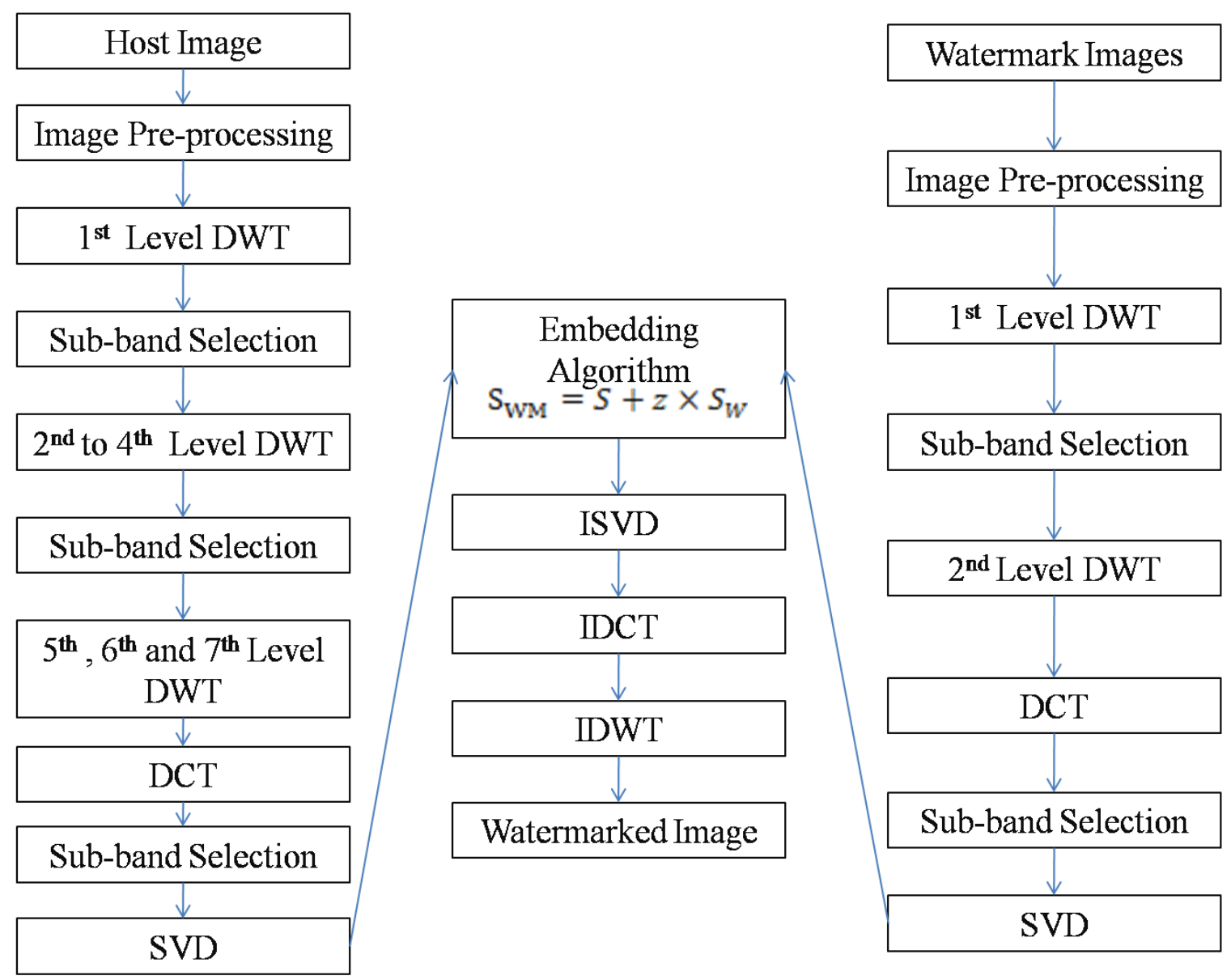

Fig. 1.1: Flowchart of the Embedding Procedure of the Proposed Technique.

\begin{tabular}{|c|c|c|c|}
\hline LL1 & & $\mathrm{H} 1$ & \\
\hline \multirow{3}{*}{ HL 1} & LL2 & \multicolumn{2}{|c|}{ LH2 } \\
\hline & \multirow{2}{*}{ HL2 } & LL3 & LH3 \\
\hline & & HL3 & \begin{tabular}{|l|l|} 
LL4 & LH4 \\
HL4 & HH4 \\
\end{tabular} \\
\hline
\end{tabular}

Fig. 1.2: Decomposition of Host Image into Sub-bands. 


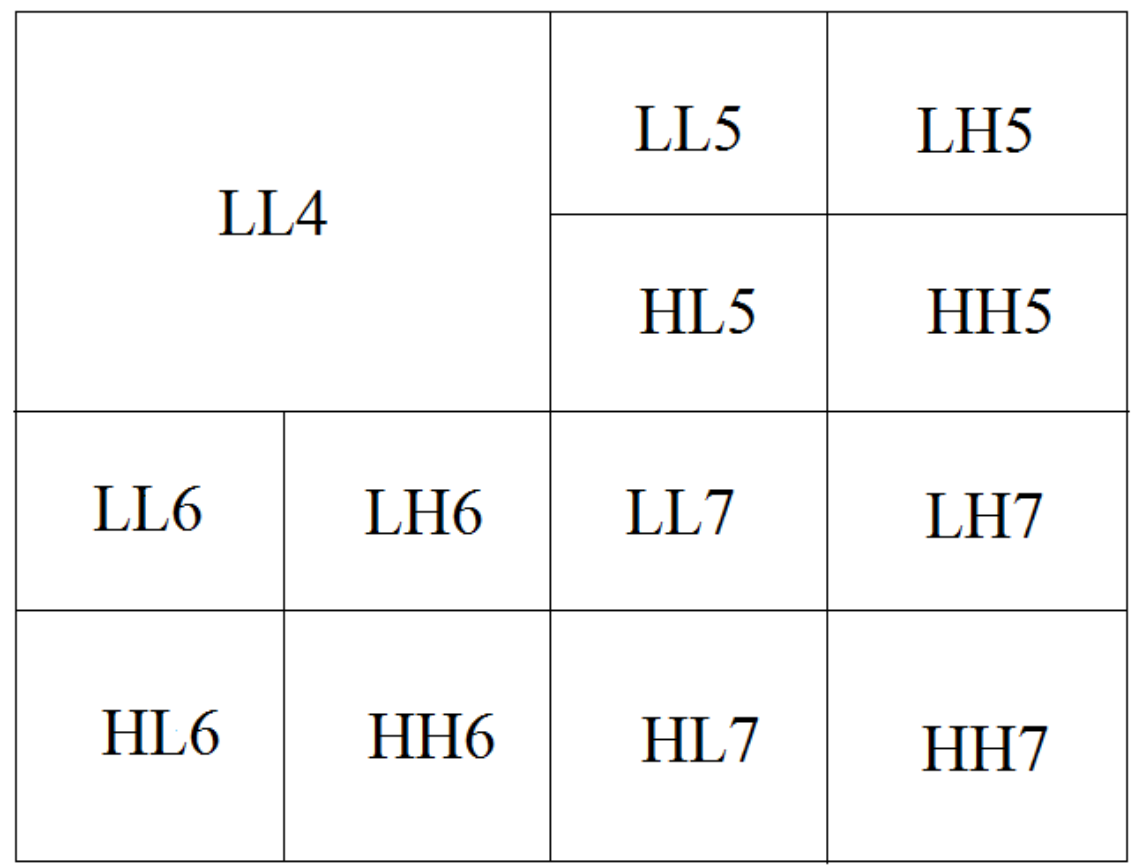

Fig. 1.3: Further Decomposition of Host Image to Position the Sub-Bands of the Watermark Images.

4. Then apply DCT (Discrete Cosine Transform) to every sub-band achieved by every level of decomposition using DWT.

5. Further, apply SVD (Singular Value Decomposition) to the sub-bands achieved by the $2^{\text {nd }}$ level of decomposition using DWT.

\section{The composition of Host image and watermark images:}

1. Combine singular values of LL5 sub-band of host image achieved by $5^{\text {th }}$ level decomposition using DWT after applying DCT to that sub-band with the singular values of HLW2 sub-band of $1^{\text {st }}$ watermark image achieved by $2^{\text {nd }}$ level decomposition using DWT after applying DCT to that sub-band.

2. Then combine singular values of LH5 sub-band of host image achieved by $5^{\text {th }}$ level decomposition using DWT after applying DCT to that sub-band with the singular values of HHW2 sub-band of $1^{\text {st }}$ watermark image achieved by $2^{\text {nd }}$ level decomposition using DWT after applying DCT to that sub-band.

3. Further, combine singular values of HL5 sub-band of host image achieved by $5^{\text {th }}$ level decomposition using DWT after applying DCT to that sub-band with the singular values of LLW2 sub-band of $1^{\text {st }}$ watermark image achieved by $2^{\text {nd }}$ level decomposition using DWT after applying DCT to that sub-band. 
4. Finally, combine singular values of HH5 sub-band of host image achieved by $5^{\text {th }}$ level decomposition using DWT after applying DCT to that sub-band with the singular values of LHW2 sub-band of $1^{\text {st }}$ watermark image achieved by $2^{\text {nd }}$ level decomposition using DWT after applying DCT to that sub-band.

5. The above 4 steps will be applicable to the other 2 watermark images. The only difference is that for $2^{\text {nd }}$ watermark the sub-bands achieved by applying $6^{\text {th }}$ level decomposition of host image using DWT will be applicable for the composition with the watermark image. And for the $3^{\text {rd }}$ watermark, the sub-bands achieved by applying $7^{\text {th }}$ level decomposition of host image using DWT will be applicable for the composition with the watermark image.

6. All these compositions will take place using a coefficient indicated by $z$. After these compositions, the sub-bands of the watermarked image will be got by performing inverse SVD. The mathematical expression will be as follows:

$$
\mathrm{S}_{\mathrm{WM}}=S+z \times S_{W}
$$

where $S_{W M}=$ Diagonal Matrix of Watermarked Image.

$\mathrm{S}=$ Diagonal Matrix of Host Image.

$S_{W}=$ Diagonal Matrix of Watermark Image.

\section{Creation of Watermarked Image:}

1. The sub-bands of the watermarked image will be achieved by performing ISVD (Inverse Singular Value Decomposition).

2. After getting the sub-bands of the watermarked image, apply IDCT (Inverse Discrete Cosine Transform) to every sub-bands of the watermarked image.

3. Then apply IDWT (Inverse Discrete Wavelet Transform) to finally get the watermarked image.

4. In these ways, the watermarked image is achieved without any perception of the watermark images.

Figure 1.1 represents the embedding technique of the first technique in a step by step diagram.

Figures $1.2 \& 1.3$ describe the way in which the host image is decomposed to locate the subbands of the watermark images. Figure 1.4 demonstrates the extraction procedure.

\subsubsection{Extraction Procedure}


1. Apply DCT to the watermarked image.

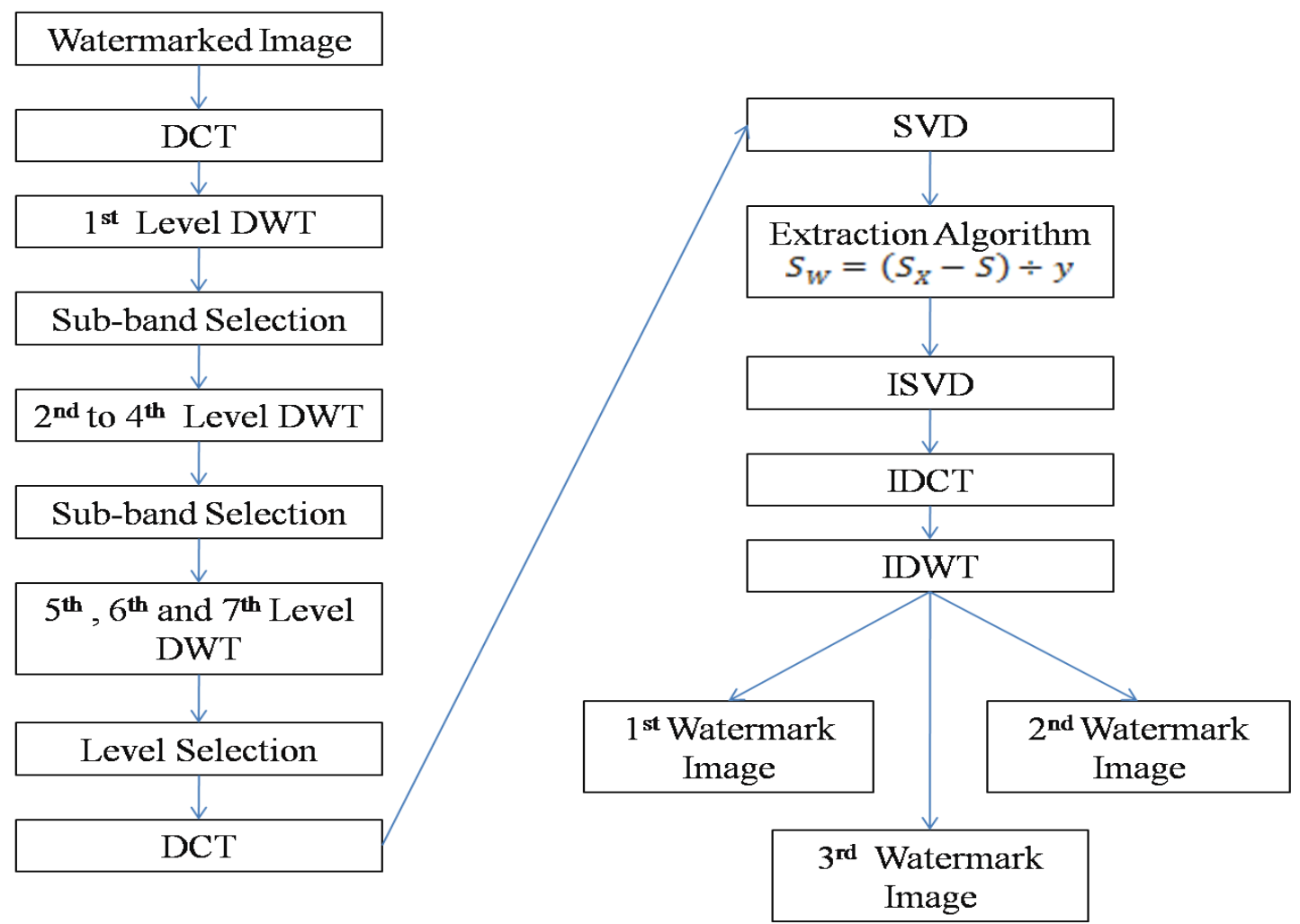

Fig.1.4: Flowchart of the Extraction Procedure of the Proposed Technique.

2. Then apply 1st level DWT (Discrete Wavelet Transform) on the image got through procedure number 1 and decompose it into LLX1, LHX1, HLX1, and HHX1 sub-bands.

3. Further apply $2^{\text {nd }}, 3^{\text {rd }}$ and $4^{\text {th }}$ level DWT on HH sub-bands which are got from $1^{\text {st }}, 2^{\text {nd }}$ and $3^{\text {rd }}$ level of decomposition respectively and get LLX2, LHX2, HLX2 \& HHX2 sub-bands, LLX3, LHX3, HLX3 \& HHX3 sub-bands and LLX4, LHX4, HLX4 \& HHX4 sub-bands from $2^{\text {nd }}, 3^{\text {rd }}$ and $4^{\text {th }}$ level DWT respectively.

4. $5^{\text {th }}$ level decomposition will be got by decomposing the LH sub-band to another 4 sub-bands which was got by the $4^{\text {th }}$ level of decomposition of the watermarked image and get LLX5, LHX5, HLX5, and HHX5 sub-bands.

5. $6^{\text {th }}$ level decomposition will be got by decomposing the HL sub-band to another 4 sub-bands which was got by the $4^{\text {th }}$ level of decomposition of the watermarked image and get LLX6, LHX6, HLX6, and HHX6 sub-bands.

6. $7^{\text {th }}$ level decomposition will be got by decomposing the HH sub-band to another 4 sub-bands which was got by the $4^{\text {th }}$ level of decomposition of the watermarked image and get LLX7, LHX7, HLX7, and HHX7 sub-bands. 
7. Then apply DCT to every sub-band got through the $5^{\text {th }}, 6^{\text {th }}$ and $7^{\text {th }}$ level of decomposition.

8. Further, apply SVD to the sub-bands got through the $5^{\text {th }}, 6^{\text {th }}$ and $7^{\text {th }}$ level of decomposition after applying DCT to those sub-bands.

9. The singular values of the watermark images will be got by the following mathematical expression:

$$
S_{W}=\left(S_{X}-S\right) \div y
$$

where $\mathrm{S}_{\mathrm{W}}=$ Diagonal Matrix of Watermark Image.

$\mathrm{S}=$ Diagonal Matrix of Host Image.

$\mathrm{S}_{\mathrm{X}}=$ Diagonal Matrix of Watermarked Image.

10. The LL, LH, HL and HH sub-bands of the watermark images will be got by applying inverse SVD of the sub-bands of the watermarked image got through $5^{\text {th }}, 6^{\text {th }}$ and $7^{\text {th }}$ level of DWT of the watermarked image.

11. Then apply inverse DCT to the sub-bands got through procedure number 10 .

12. Further, apply inverse DWT to the sub-bands got through procedure number 10 after applying inverse DCT to those sub-bands.

In these ways, the three watermark images are achieved without any distortion caused to those images.

In figures 1.1 and 1.4, image pre-processing means resize the host and the mark images to the suitable size so that the proposed technique can be implemented successfully. By image preprocessing any kind of images can be used. Those images should only be converted into suitable size defined for different watermarking techniques. In the flow chart of embedding technique, sub-band selection means which sub-band is selected for higher level of decomposition. In our proposed technique, HH sub-band was decomposed several times to get the desired level of decomposition. After $4^{\text {th }}$ level of decomposition again sub-band selection was done to get $5^{\text {th }}, 6^{\text {th }}$ and $7^{\text {th }}$ level of decomposition. Then DCT was done. After DCT, again sub-band selection was done to know on which sub-bands SVD will be applied. After that, embedding algorithm was done and finally after doing some inverse function operation, the watermarked image was obtained. In the flow chart of extraction operation, after $5^{\text {th }}, 6^{\text {th }}$ and $7^{\text {th }}$ level of decomposition, level selection was done as DCT was applied to the sub-bands obtained by $5^{\text {th }}, 6^{\text {th }}$ and $7^{\text {th }}$ level of decomposition. Then SVD was done. After that, extraction algorithm and some other operations were done to get the mark images. 


\subsubsection{Performance Analysis}

A variety of experiments have been carried out to check the feasibility of the proposed method. A $512 \times 512$ "Lena" image was used as the host image and three $512 \times 512$ images were used as watermark images.

Peak Signal to Noise Ratio (PSNR) is employed to measure the quality of the watermarked image. In addition, Normalized Correlation (NC) is exploited to check the similarity between the watermark images and the extracted watermarks from the attacked image. Table 1.1 indicates the value of PSNR for different values of $z$. Also, the value of NC between the host image and the watermarked image was evaluated for different values of $z$. Figures 1.5 and 1.6 graphically represent the results obtained from table 1.1.

TABLE 1.1 THE CHANGE OF PSNR AND NC FOR DIFFERENT VALUES OF $\mathbf{z}$

\begin{tabular}{|c|c|c|}
\hline $\begin{array}{c}\text { Value of Co-efficient for } \\
\text { Embedding Mark Images } \\
z\end{array}$ & $\begin{array}{c}\text { PSNR for Watermarked } \\
\text { Image }\end{array}$ & $\begin{array}{c}\text { Value of NC between } \\
\text { the Host Image and } \\
\text { the Watermarked } \\
\text { Image }\end{array}$ \\
\hline 0.0001 & 108.3781 & 0.9999 \\
\hline 0.001 & 108.3781 & 0.9999 \\
\hline 0.002 & 108.3780 & 0.9999 \\
\hline 0.003 & 108.3780 & 0.9999 \\
\hline 0.004 & 108.3780 & 0.9999 \\
\hline 0.005 & 108.3779 & 0.9999 \\
\hline 0.006 & 108.3779 & 0.9999 \\
\hline 0.007 & 108.3778 & 0.9999 \\
\hline 0.008 & 108.3777 & 0.9999 \\
\hline 0.009 & 108.3776 & 0.9999 \\
\hline 0.01 & 108.3775 & 0.9999 \\
\hline 0.04 & 108.3685 & \\
\hline
\end{tabular}




\begin{tabular}{|c|c|c|}
\hline 0.06 & 108.3565 & 0.9999 \\
\hline 0.08 & 108.3397 & 0.9999 \\
\hline 0.1 & 108.3182 & 0.9999 \\
\hline 0.2 & 108.1405 & 0.9999 \\
\hline 0.4 & 107.4595 & 0.9999 \\
\hline 0.6 & 106.4184 & 0.9999 \\
\hline 0.8 & 105.1218 & 0.9999 \\
\hline 1 & 103.6692 & 0.9999 \\
\hline
\end{tabular}

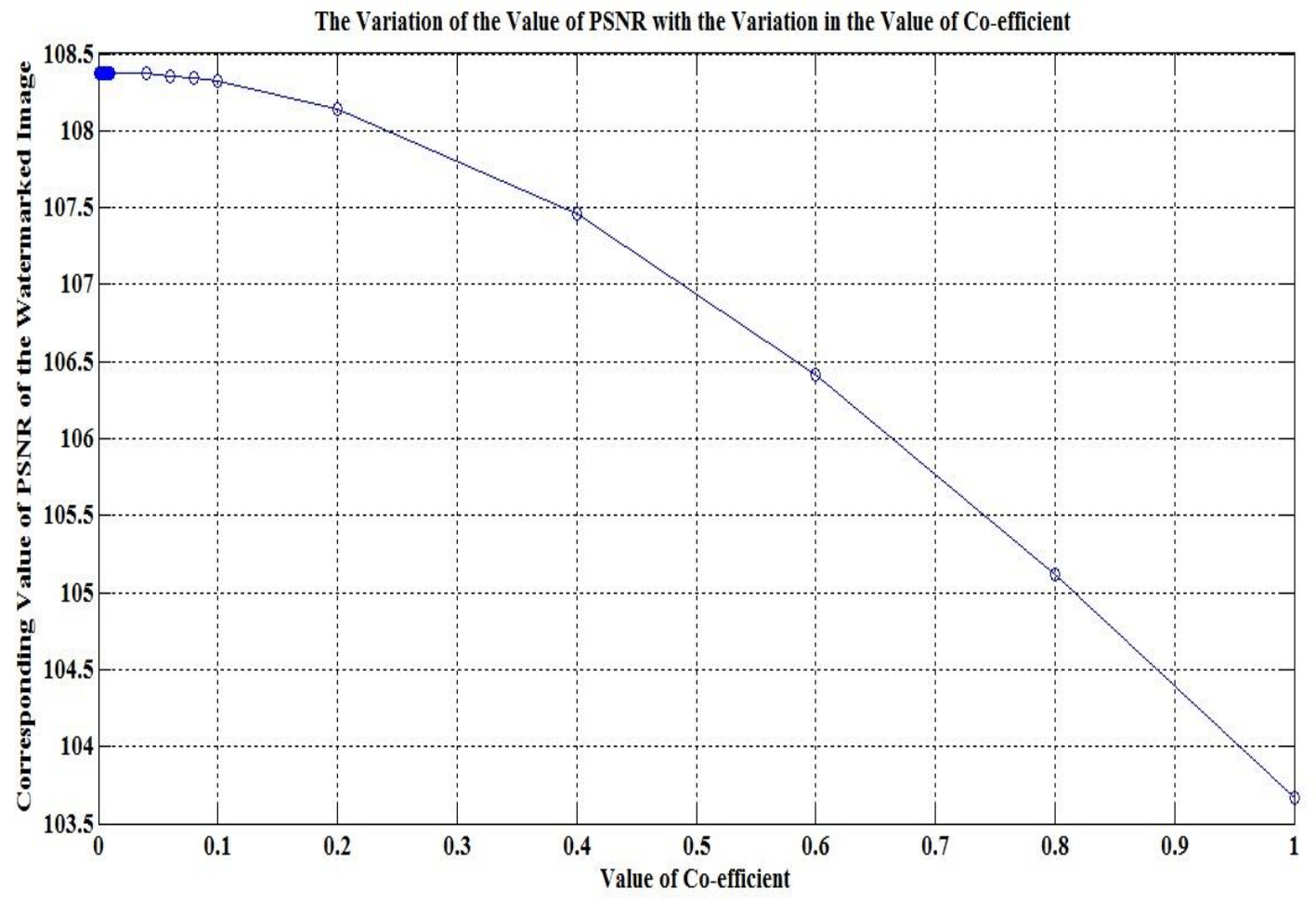

Fig. 1.5: The Variation in the Value of PSNR with the Variation in the Value of $z$. 


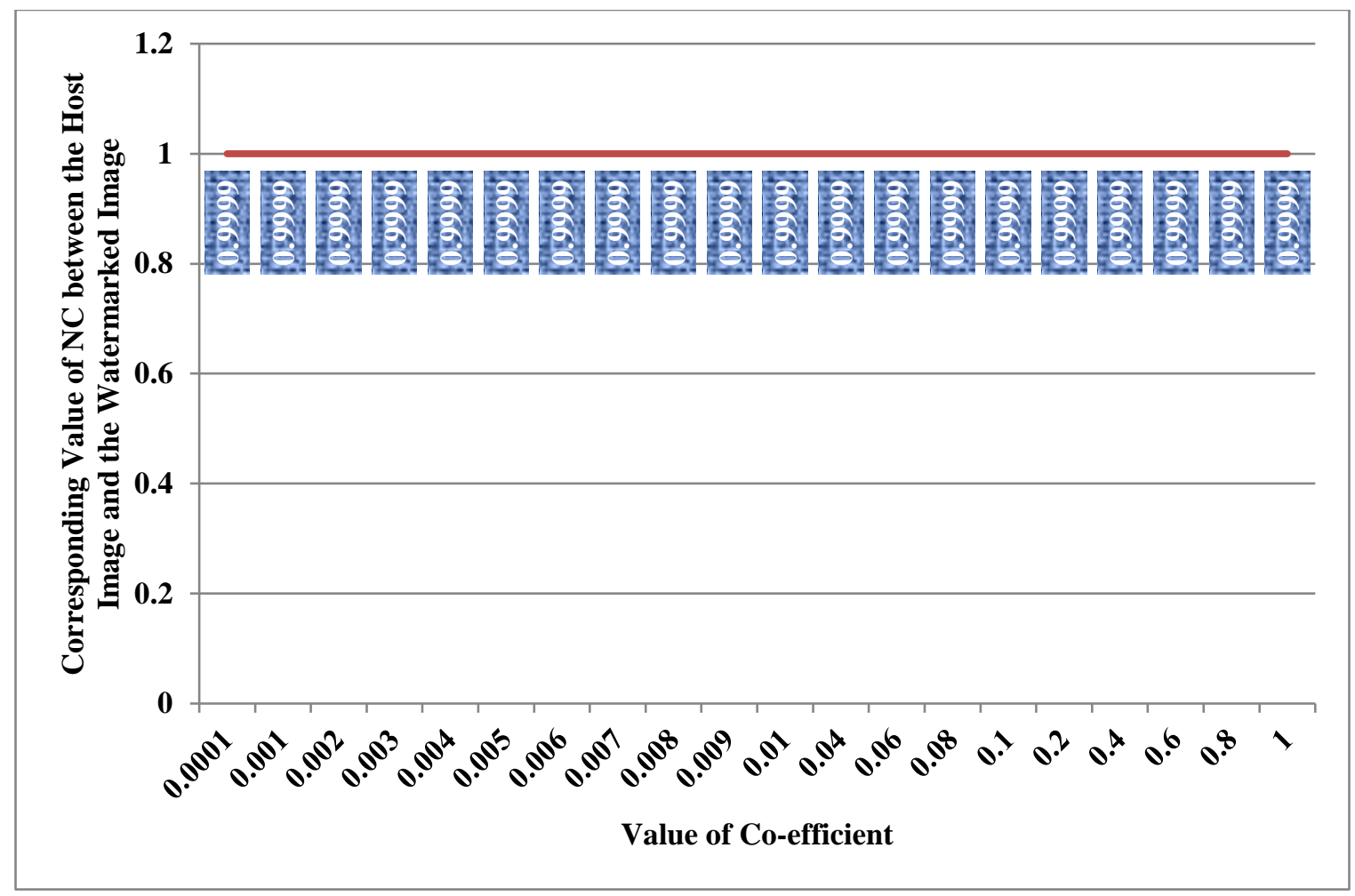

Fig. 1.6: The Variation in the Value of $\mathrm{NC}$ with the Variation in the Value of $\mathbf{z}$.

From table 1.1, it is evident that the best result of PSNR can be obtained by taking the value of $z$ equal to 0.001. For that value, the PSNR and NCC (Normalized Cross Correlation) of the mark images will be calculated by changing the value of coefficient for extracting the mark images which is $\boldsymbol{y}$. Table 1.2 indicates those values. Figures 1.7 to 1.12 graphically represent the results obtained from table 3.2.

\section{TABLE 1.2 THE CHANGE OF PSNR AND NCC OF THE MARK IMAGES FOR DIFFERENT VALUES OF $\mathbf{y}$}

\begin{tabular}{|c|c|c|c|c|c|c|}
\hline $\begin{array}{c}\text { Value of Co- } \\
\text { efficient for } \\
\begin{array}{c}\text { Extracting Mark } \\
\text { Images } \\
y\end{array}\end{array}$ & \multicolumn{3}{|c|}{ PSNR for Mark Images } & \multicolumn{3}{c|}{$\begin{array}{c}\text { Value of NCC of Mark Images } \\
\text { between before Embedded and after } \\
\text { Extracted }\end{array}$} \\
\cline { 2 - 7 } & $\begin{array}{c}\text { 1st } \\
\text { Watermark }\end{array}$ & $\begin{array}{c}\text { 2nd } \\
\text { Watermark }\end{array}$ & $\begin{array}{c}\text { 3rd } \\
\text { Watermark }\end{array}$ & $\begin{array}{c}\text { 1st } \\
\text { Watermark }\end{array}$ & $\begin{array}{c}\text { 2nd } \\
\text { Watermark }\end{array}$ & $\begin{array}{c}3 \text { rd } \\
\text { Watermark }\end{array}$ \\
\hline 0.001 & -103.9896 & -84.0055 & -90.1894 & 0.0189 & 0.2587 & 0.4114 \\
\hline 0.01 & -57.9348 & -37.9515 & -44.1168 & 0.2645 & 0.2583 & 0.4114 \\
\hline 0.1 & -11.8529 & 8.1185 & 2.1367 & 0.2648 & 0.2570 & 0.4111 \\
\hline 1 & 34.4257 & 53.8857 & 49.3460 & 0.7004 & 0.8136 & 0.9590 \\
\hline
\end{tabular}




\begin{tabular}{|c|c|c|c|c|c|c|}
\hline 2 & 48.3740 & 66.5358 & 62.3466 & 0.8901 & 0.9347 & 0.9882 \\
\hline 4 & 61.8736 & 76.3313 & 70.2148 & 0.9674 & 0.9740 & 0.9946 \\
\hline 6 & 68.9818 & 79.8855 & 71.7431 & 0.9835 & 0.9816 & 0.9953 \\
\hline 8 & 73.2664 & 81.4531 & 72.0107 & 0.9892 & 0.9843 & 0.9955 \\
\hline 10 & 75.9813 & 82.2465 & 71.9888 & 0.9917 & 0.9855 & 0.9954 \\
\hline 14 & 78.9420 & 82.9608 & 71.7966 & 0.9938 & 0.9865 & 0.9954 \\
\hline 18 & 80.3268 & 83.2505 & 71.6159 & 0.9946 & 0.9869 & 0.9953 \\
\hline 20 & 80.7345 & 83.3319 & 71.5409 & 0.9948 & 0.9870 & 0.9952 \\
\hline 21 & 80.8942 & 83.3634 & 71.5069 & 0.9949 & 0.9870 & 0.9952 \\
\hline 24 & 81.2498 & 83.4335 & 71.4173 & 0.9951 & 0.9871 & 0.9952 \\
\hline 28 & 81.5374 & 83.4907 & 71.3211 & 0.9952 & 0.9872 & 0.9951 \\
\hline 30 & 81.6319 & 83.5099 & 71.2809 & 0.9953 & 0.9872 & 0.9951 \\
\hline 32 & 81.7045 & 83.5251 & 71.2449 & 0.9953 & 0.9872 & 0.9951 \\
\hline 36 & 81.8041 & 83.5466 & 71.1831 & 0.9954 & 0.9873 & 0.9951 \\
\hline 40 & 81.8640 & 83.5607 & 71.1322 & 0.9954 & 0.9873 & 0.9950 \\
\hline
\end{tabular}

From the values of table 1.2, the value of 36 can be taken as after an increase in the value, causes a decrease in the value of NCC for the third watermark.

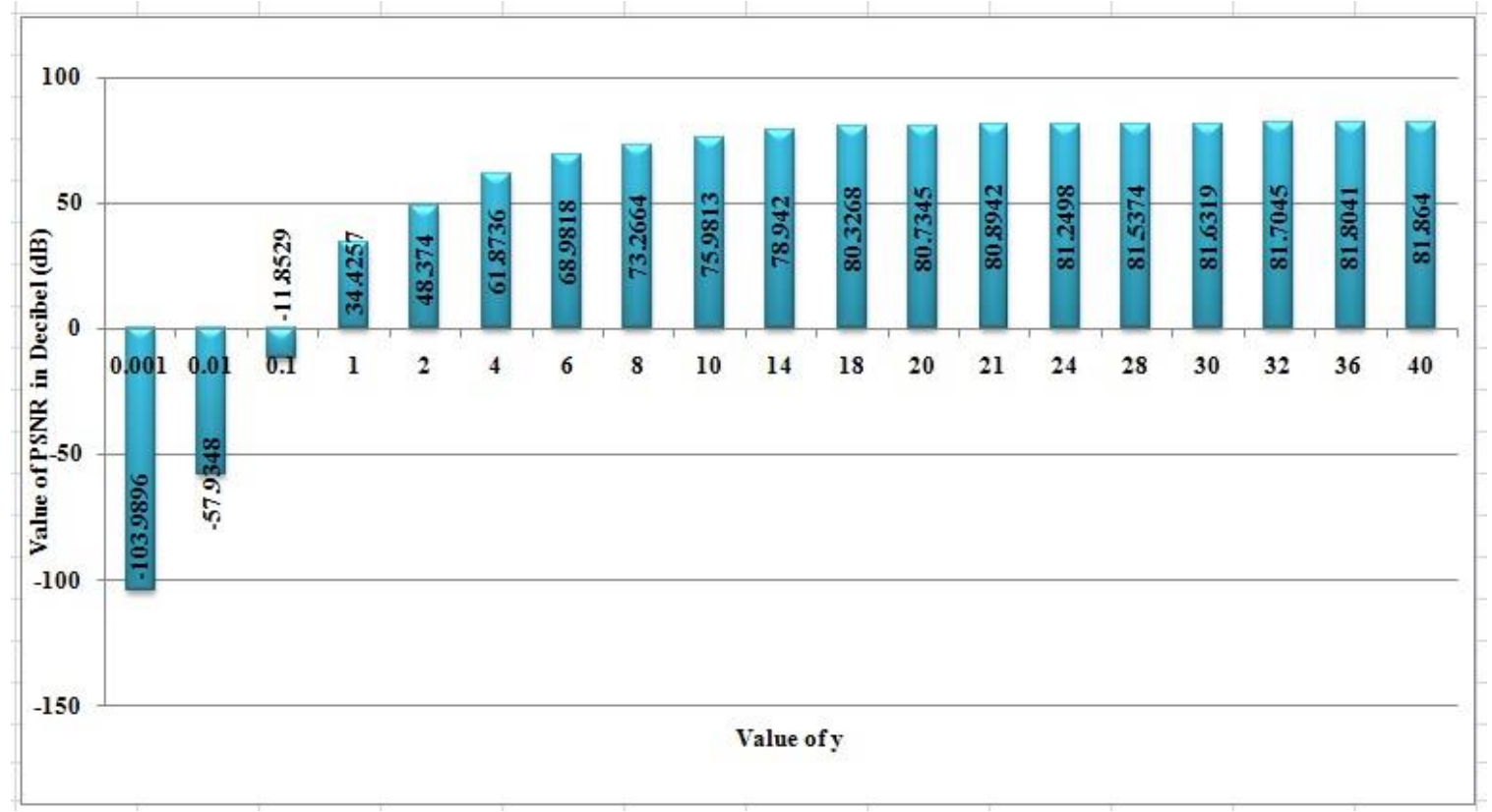

Fig. 1.7: The Variation in the Value of PSNR of Mark Image 1with the Variation in the Value of $y$. 


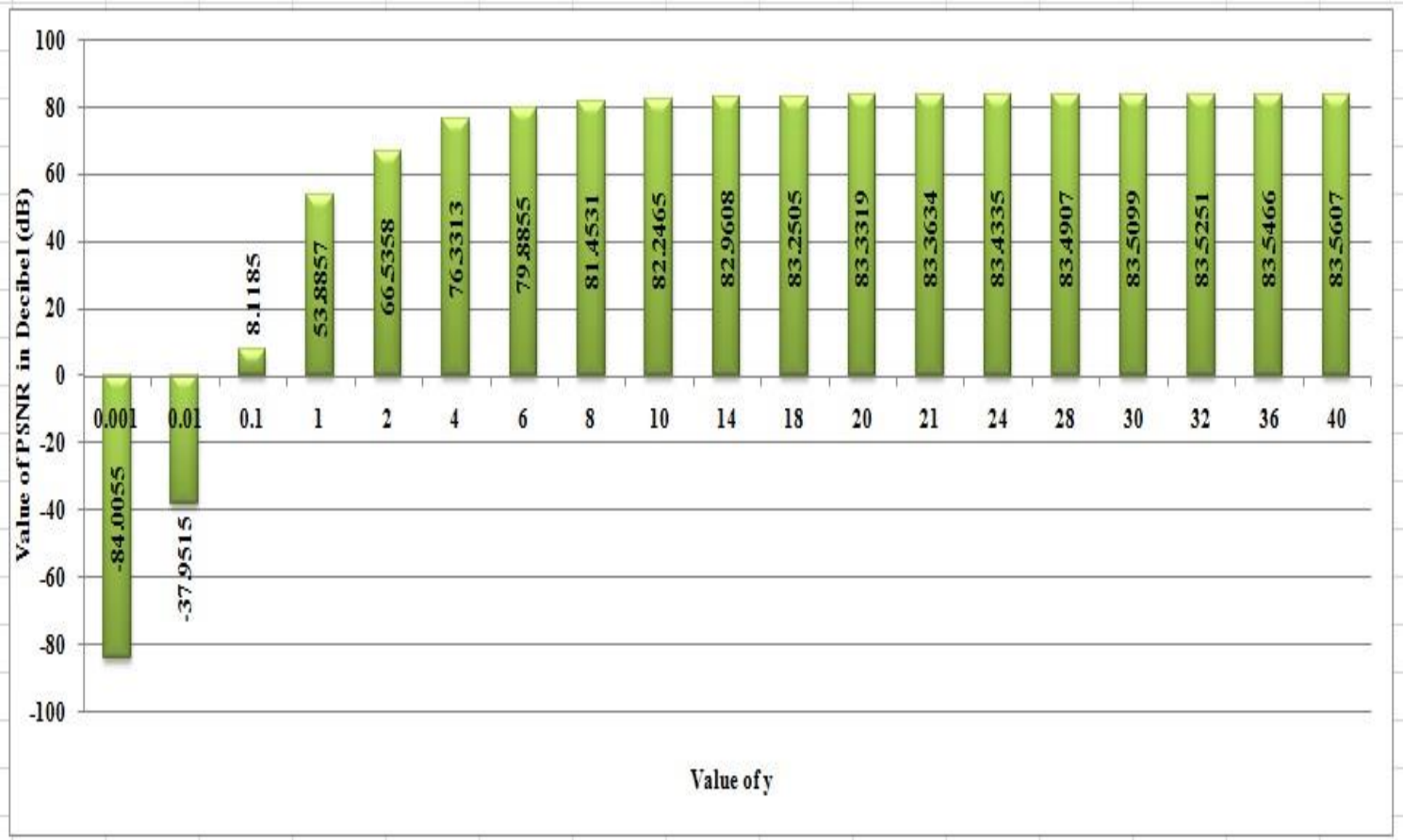

Fig. 1.8: The Variation in the Value of PSNR of Mark Image 2with the Variation in the Value of $\boldsymbol{y}$.

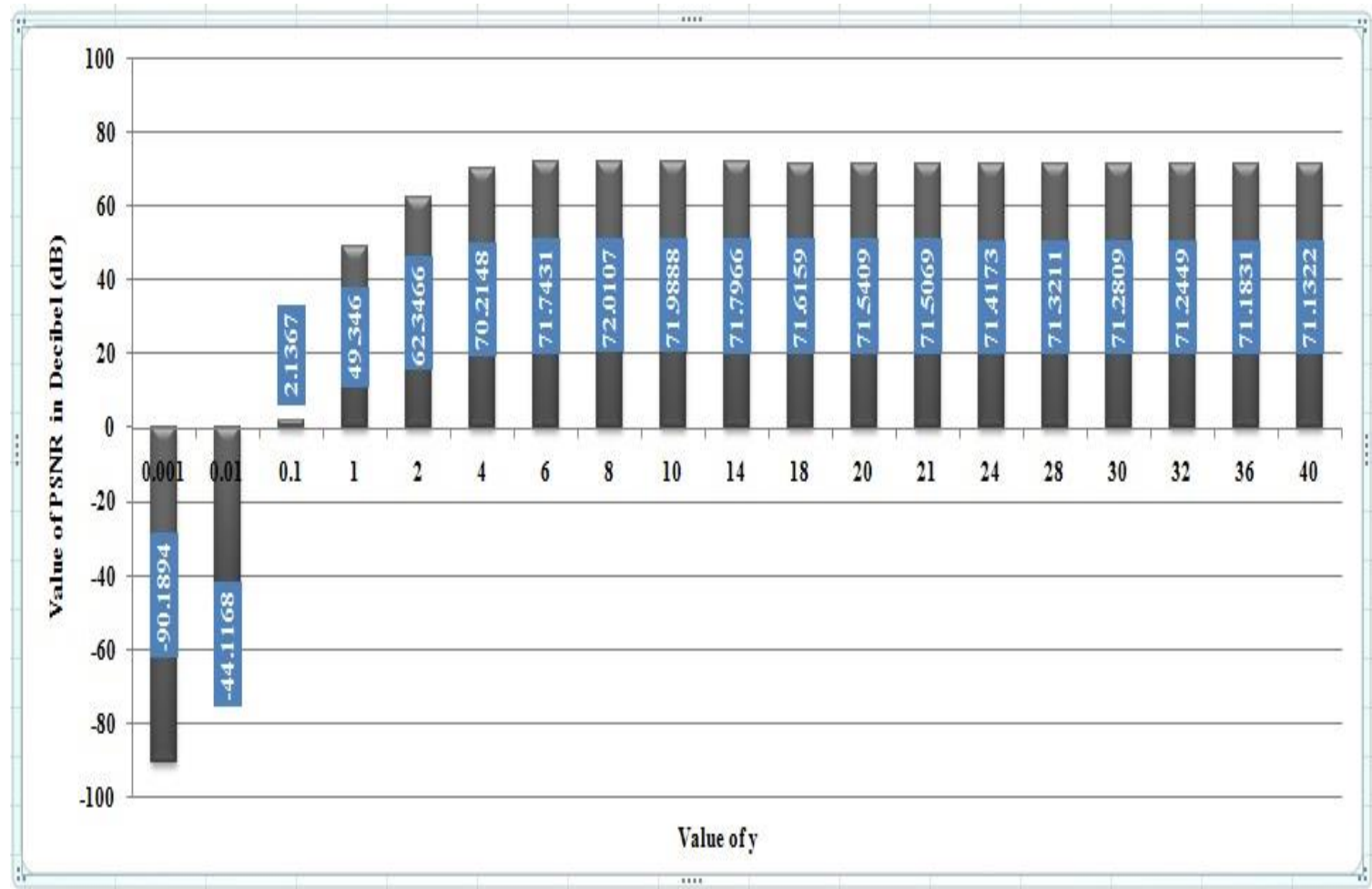

Fig. 1.9: The Variation in the Value of PSNR of Mark Image 3 with the Variation in the Value of $y$. 


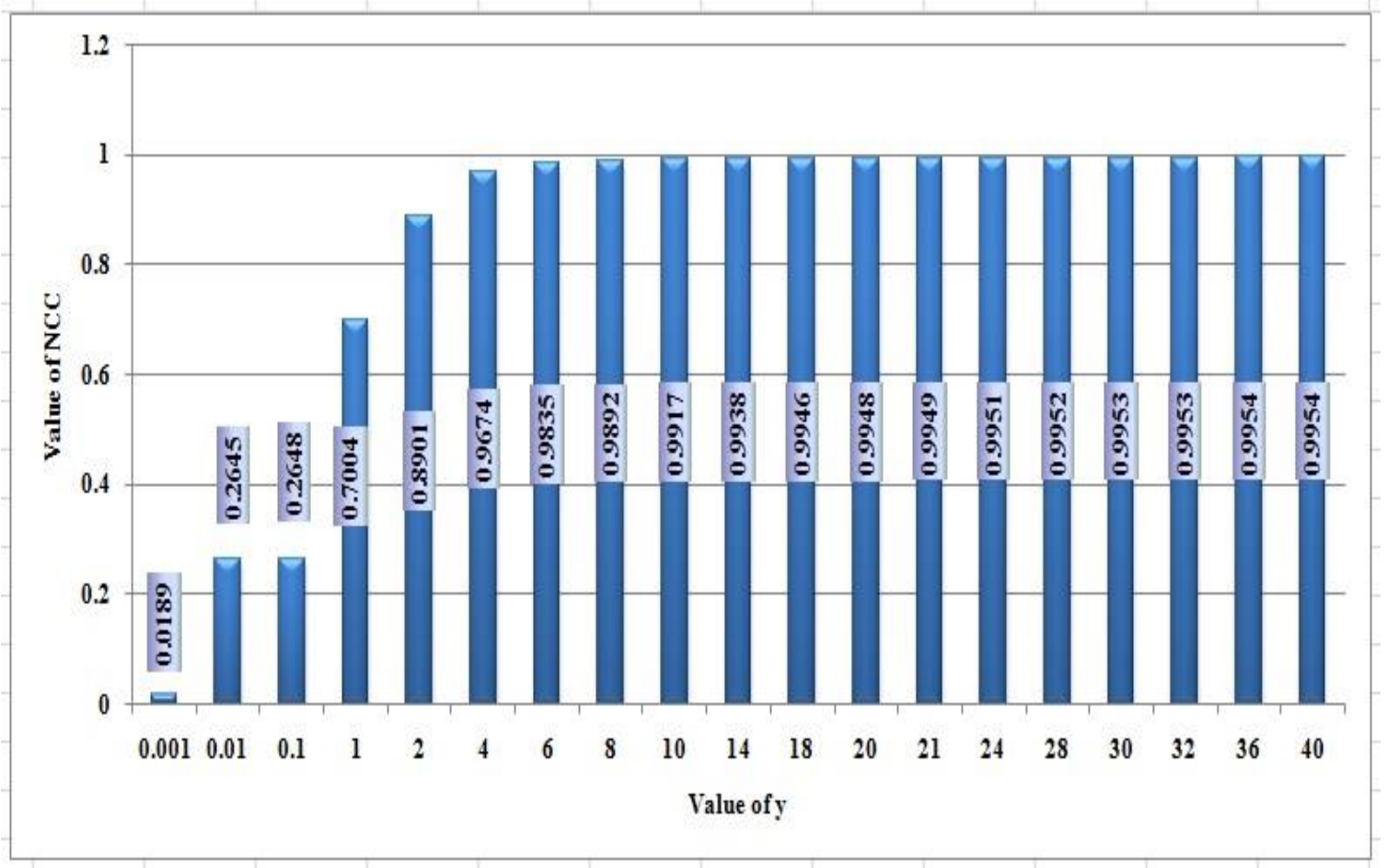

Fig. 1.10:The Variation in the Value of NCC of Mark Image 1 with the Variation in the Value of $y$.

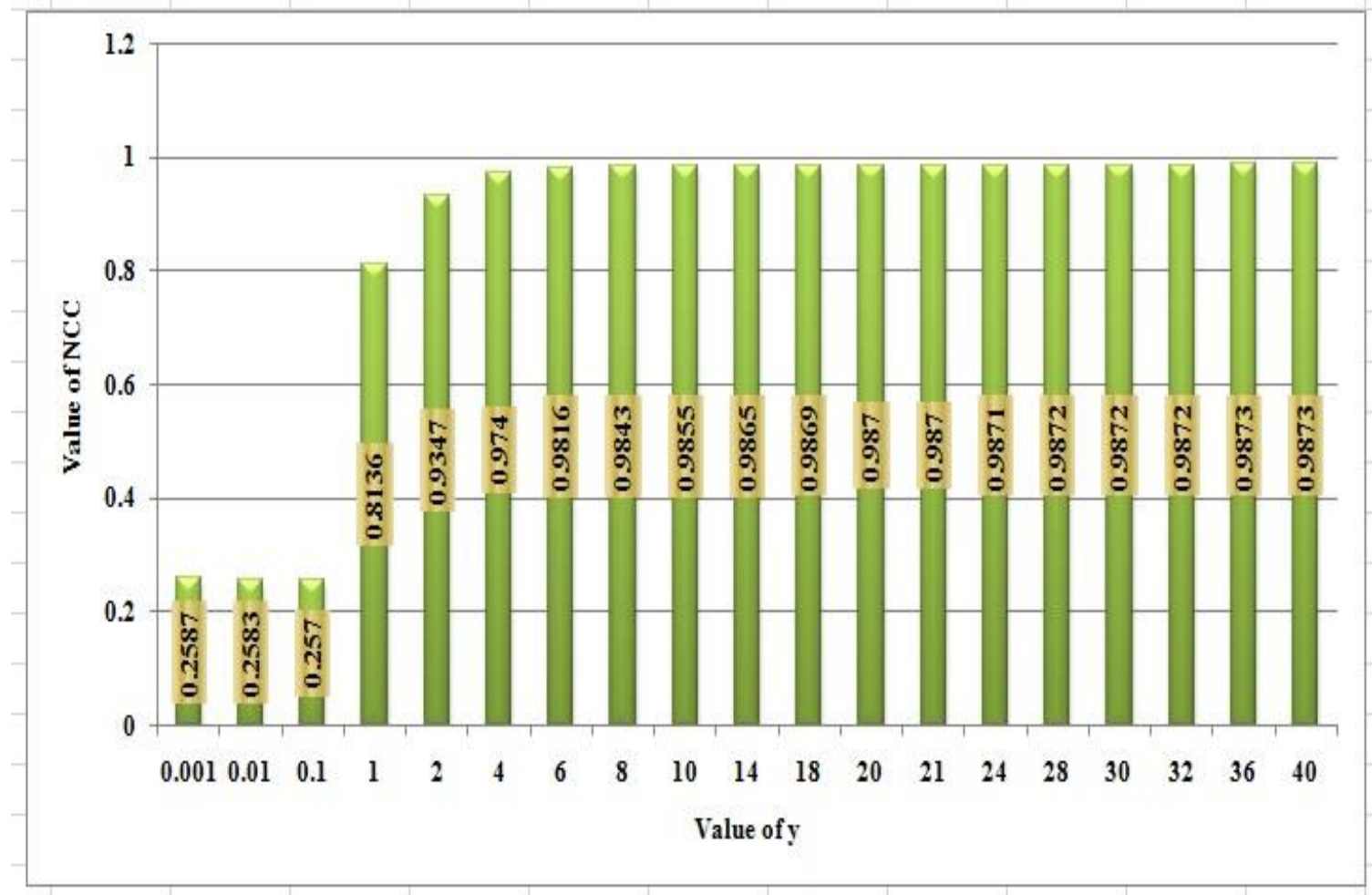

Fig. 1.11:The Variation in the Value of NCC of Mark Image 2 with the Variation in the Value of $y$. 


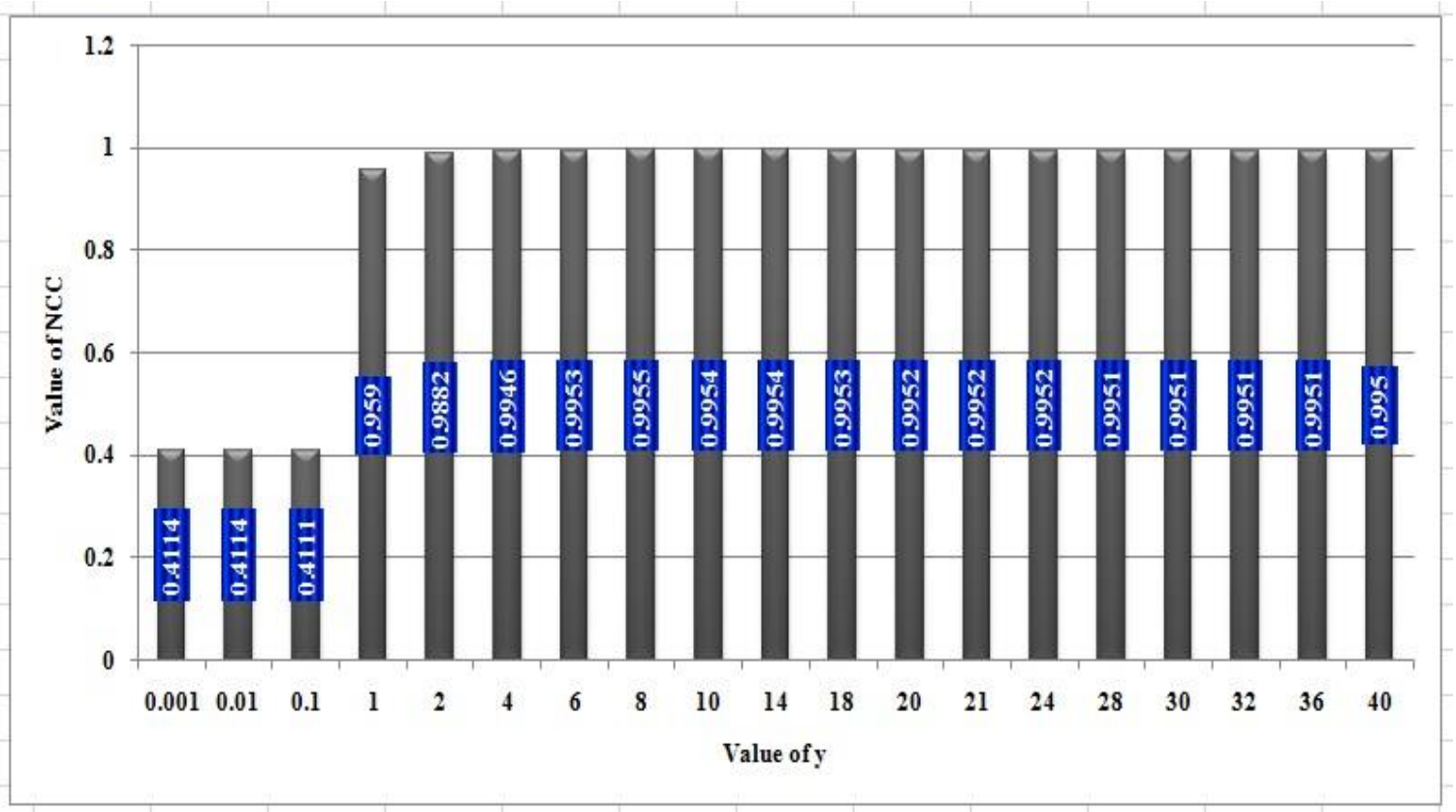

Fig. 1.12:The Variation in the Value of NCC of Mark Image 3 with the Variation in the Value of $y$.

Figure 1.13 represents the results obtained after using the proposed technique. It is evident from the figure that the proposed technique successfully recovered the watermark image and also fulfilled the invisibility of watermark images in the watermarked image which is one of the important conditions of a successful 'Digital Watermarking'.

\begin{tabular}{|c|c|c|c|}
\hline \multirow{2}{*}{$\begin{array}{lr}\text { Table } & 1.3 \\
\text { PSNR } & \text { values }\end{array}$} & Number of Watermarks & PSNR & \multirow{4}{*}{$\begin{array}{l}\text { shows the } \\
\text { of } \\
\text { image when } \\
\text { watermark, as } \\
\text { multiple }\end{array}$} \\
\hline & One Watermark & $108.3781 \mathrm{~dB}$ & \\
\hline one & Two Watermarks & $108.3781 \mathrm{~dB}$ & \\
\hline well & Three Watermarks & $108.3781 \mathrm{~dB}$ & \\
\hline
\end{tabular}

watermarks is used. To find the resistivity of the proposed methodology, the watermarked image has been gone through various attacks, such as: Salt \& Pepper noise, Gaussian noise, Speckle noise, Poisson noise, Blurring, Sharpening, Rotation, Histogram Equalization , Gaussian Lowpass Filter, Prewitt Filter, Sobel Filter, Median filter, Mean filter and JPEG Compression. Figures 1.14 and 1.15 graphically represent the values of NCC for different degrees of rotation. Figures $1.16,1.17$ and 1.18 graphically represent the resistivity of the proposed methodology by indicating the values of NC for various attacks. The values of NCC are also measured to indicate that the watermark images are not altered significantly by the process of watermarking as well as the addition of different attacks on the watermarked image. Figures 1.19-1.33 represent the 
values of PSNR of the mark images after being extracted from the watermarked image against several attacks and filtering. The method has been implemented using MATLABR2014a.

TABLE 1.3: VALUE OF PSNR FOR DIFFERENT NUMBER OF WATERMARKS

\begin{tabular}{|c|c|}
\hline Before Watermarking & After Watermarking \\
\hline Host Image & Watermarked Image \\
\hline & \\
\hline
\end{tabular}




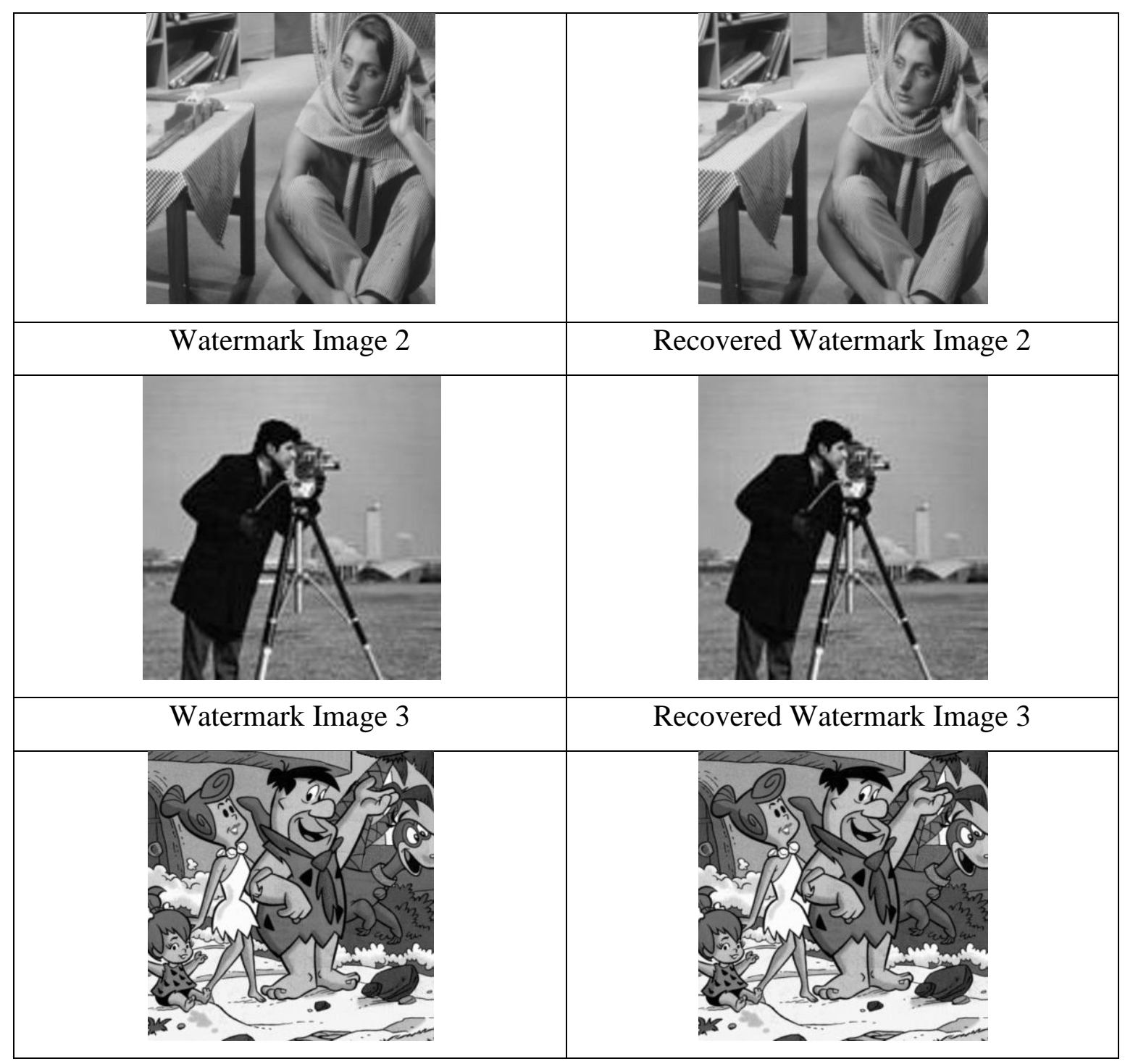

Fig.1.13: The Results Obtained After Applying 'Digital Watermarking'. 


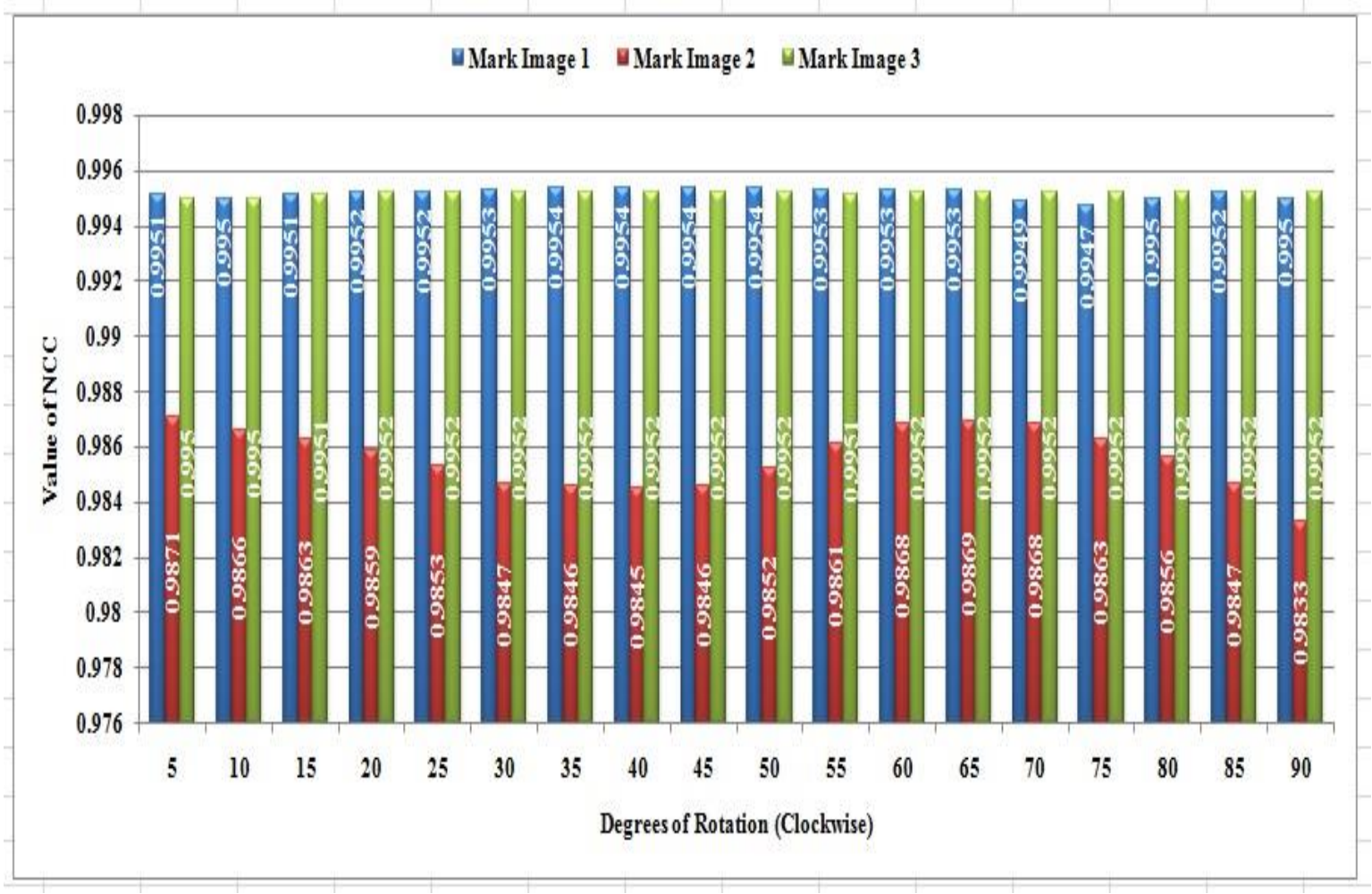

Fig. 1.14: The Variation of the Value of NCC of the Watermark Images for Different Degrees of Rotation (Clockwise).

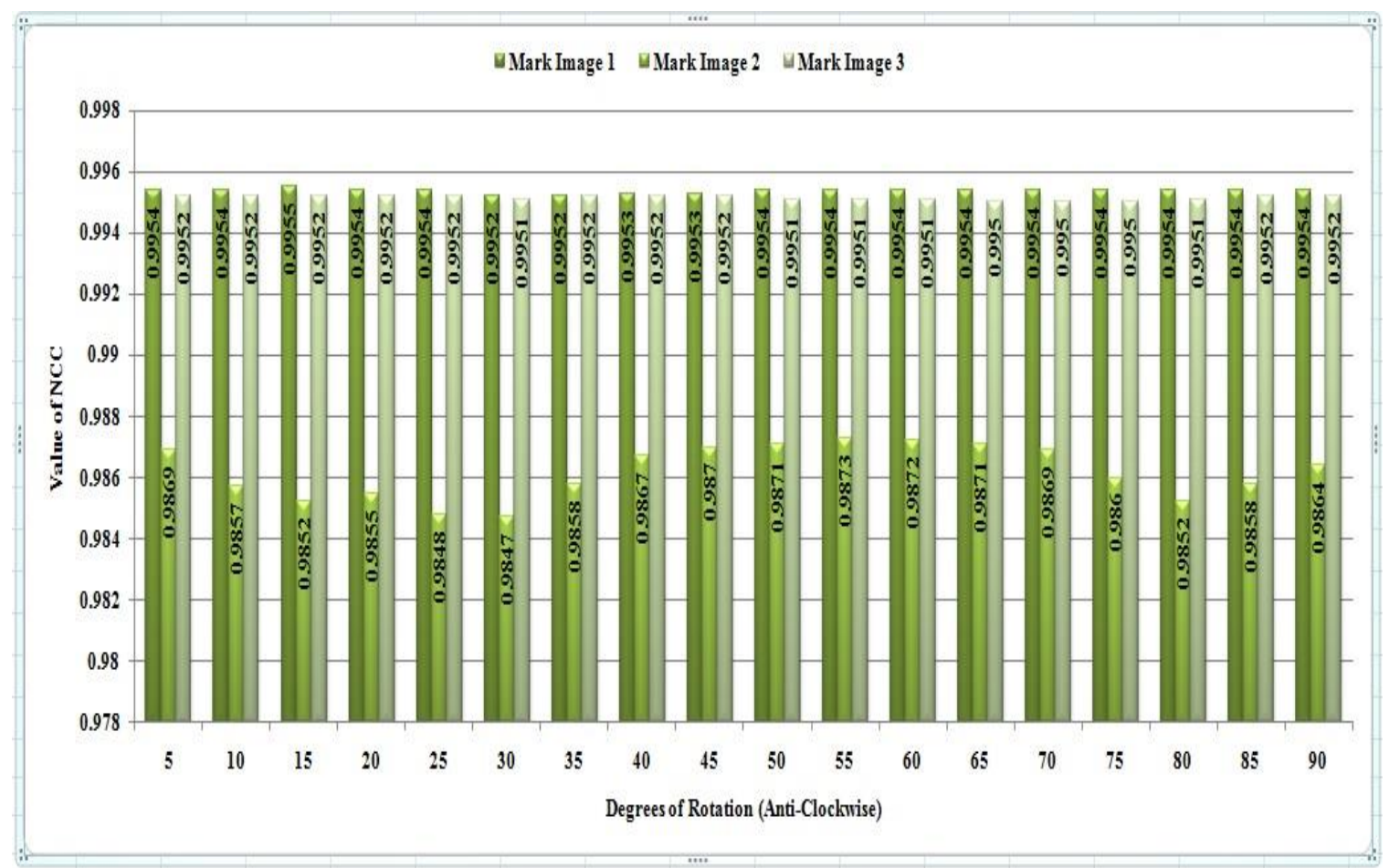

Fig. 1.15: The Variation of the Value of NCC of the Watermark Images for Different Degrees of Rotation (Anti-Clockwise). 


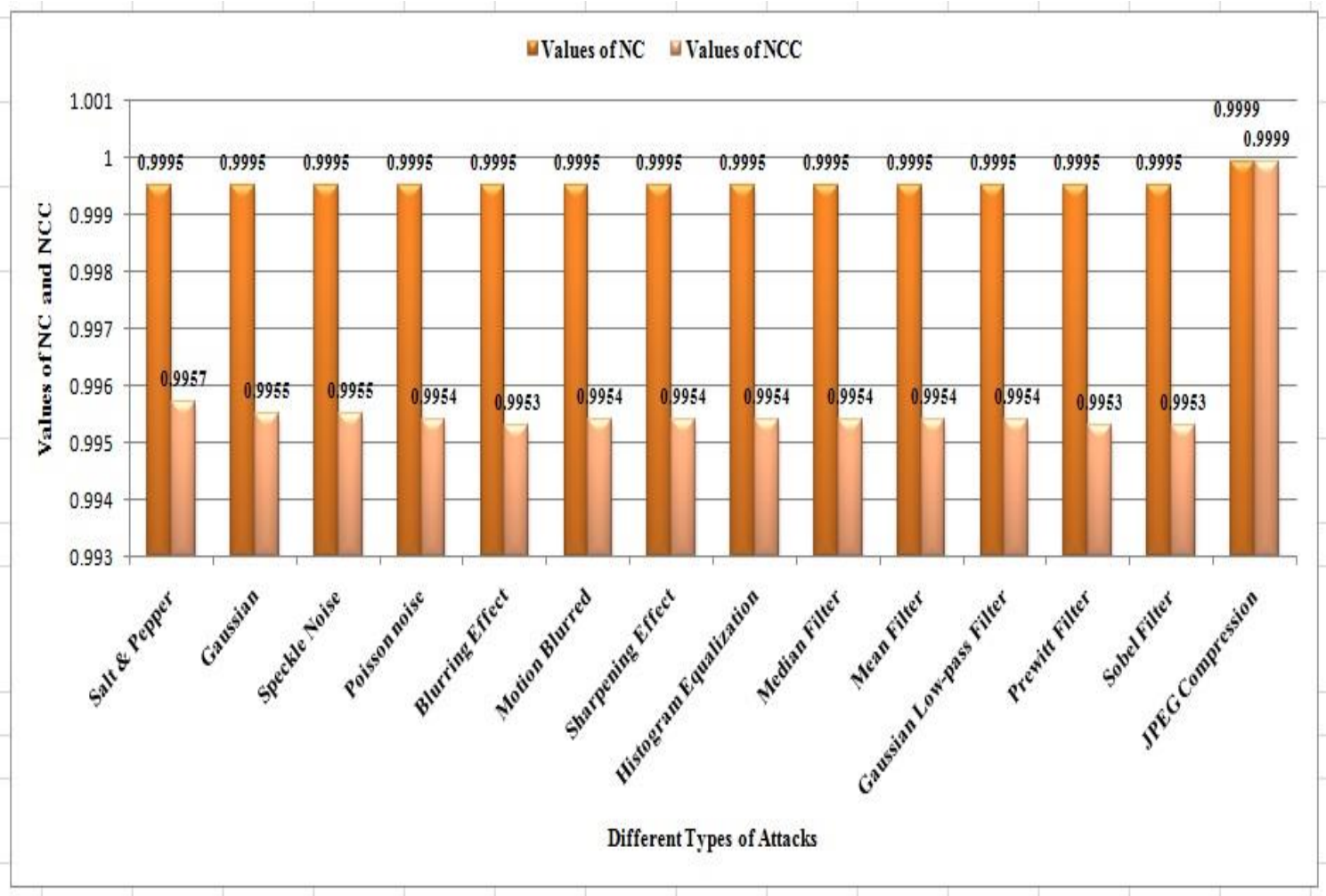

Fig.1.16: The Variation of the Value of NC and NCC of Watermark Image 1 with the Application of Several Attacks and Filtering.

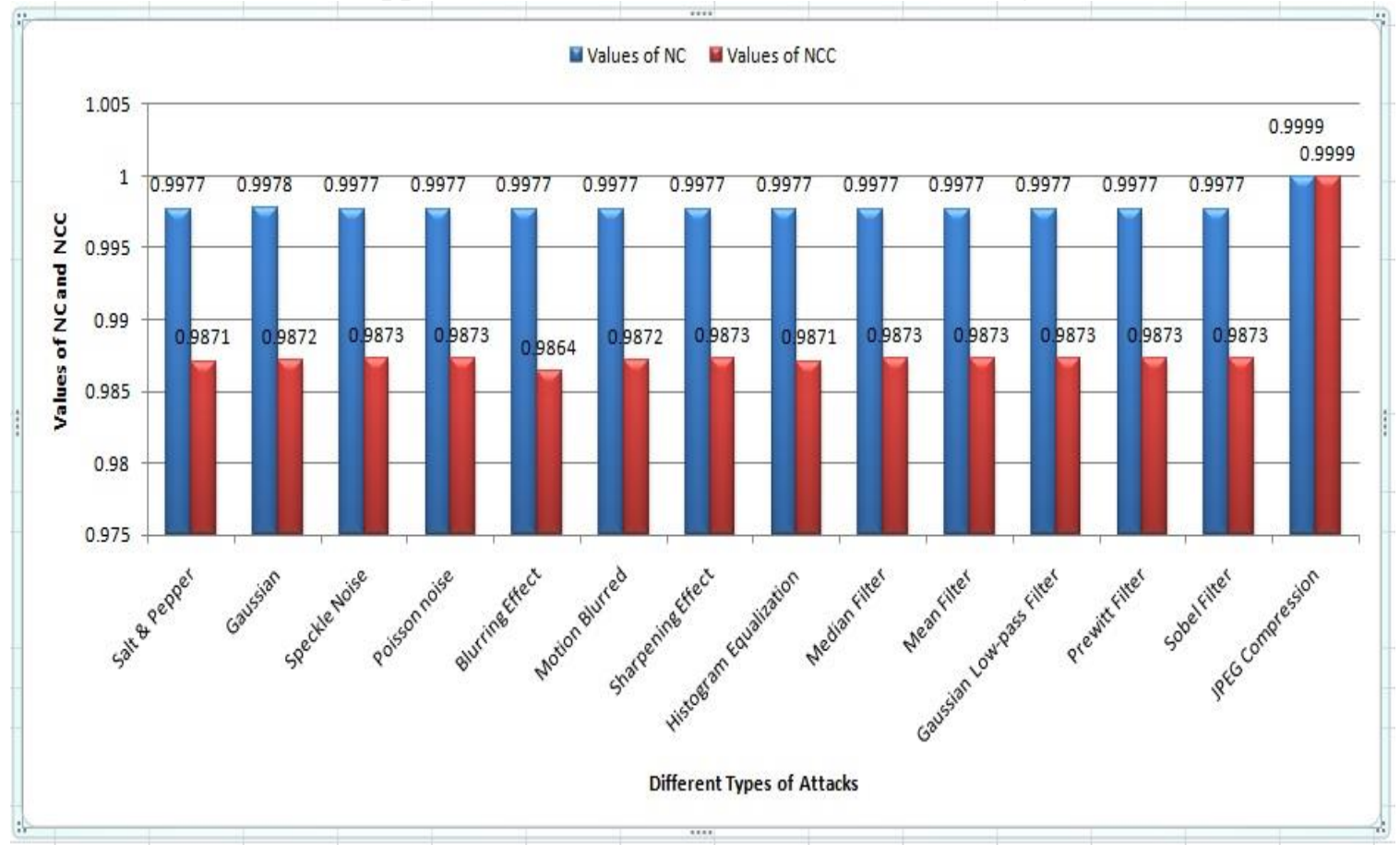

Fig.1.17: The Variation of the Value of NC and NCC of Watermark Image 2 with the Application of Several Attacks and Filtering. 


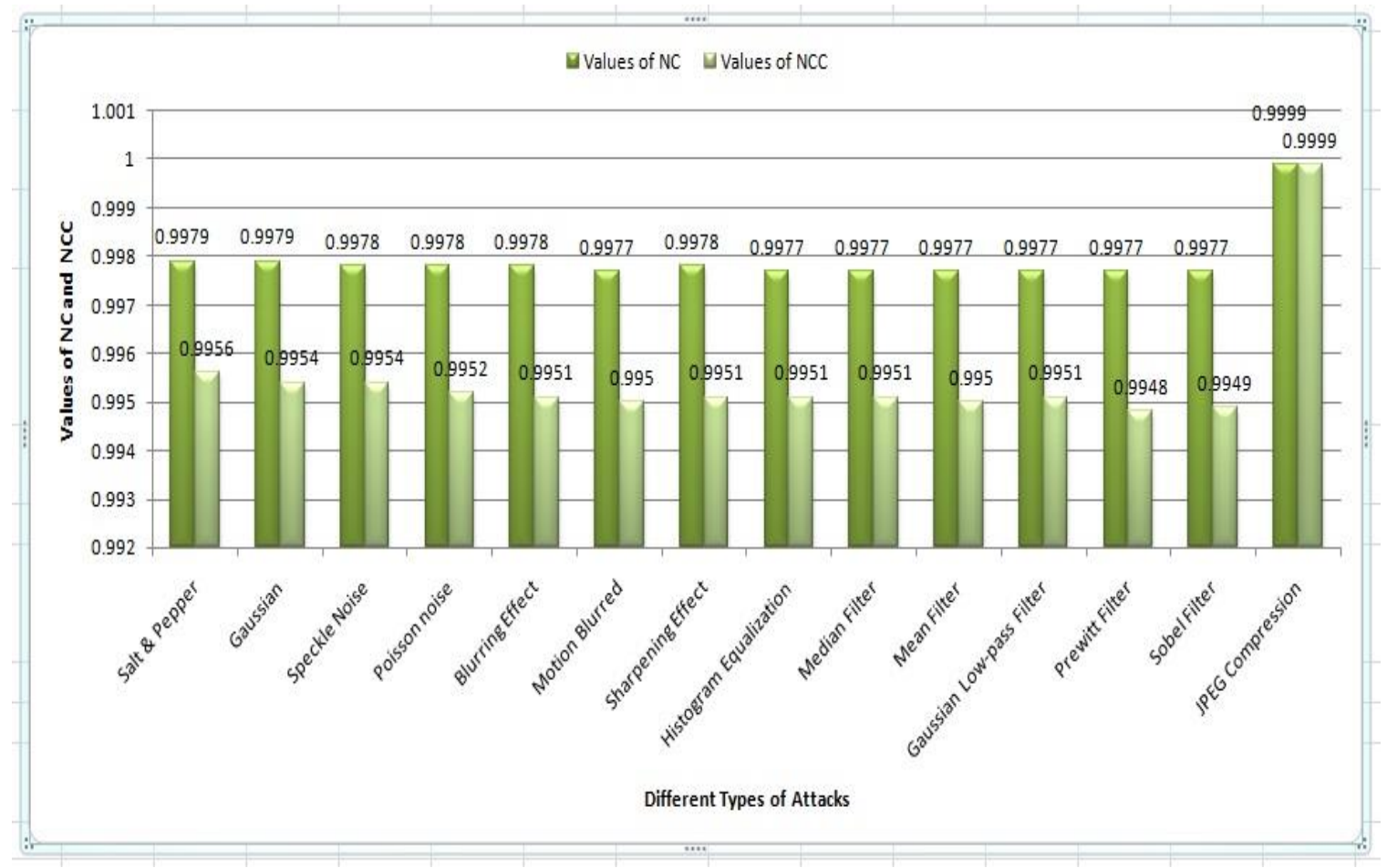

Fig.1.18: The Variation of the Value of NC and NCC of Watermark Image 3 with the Application of Several Attacks and Filtering.

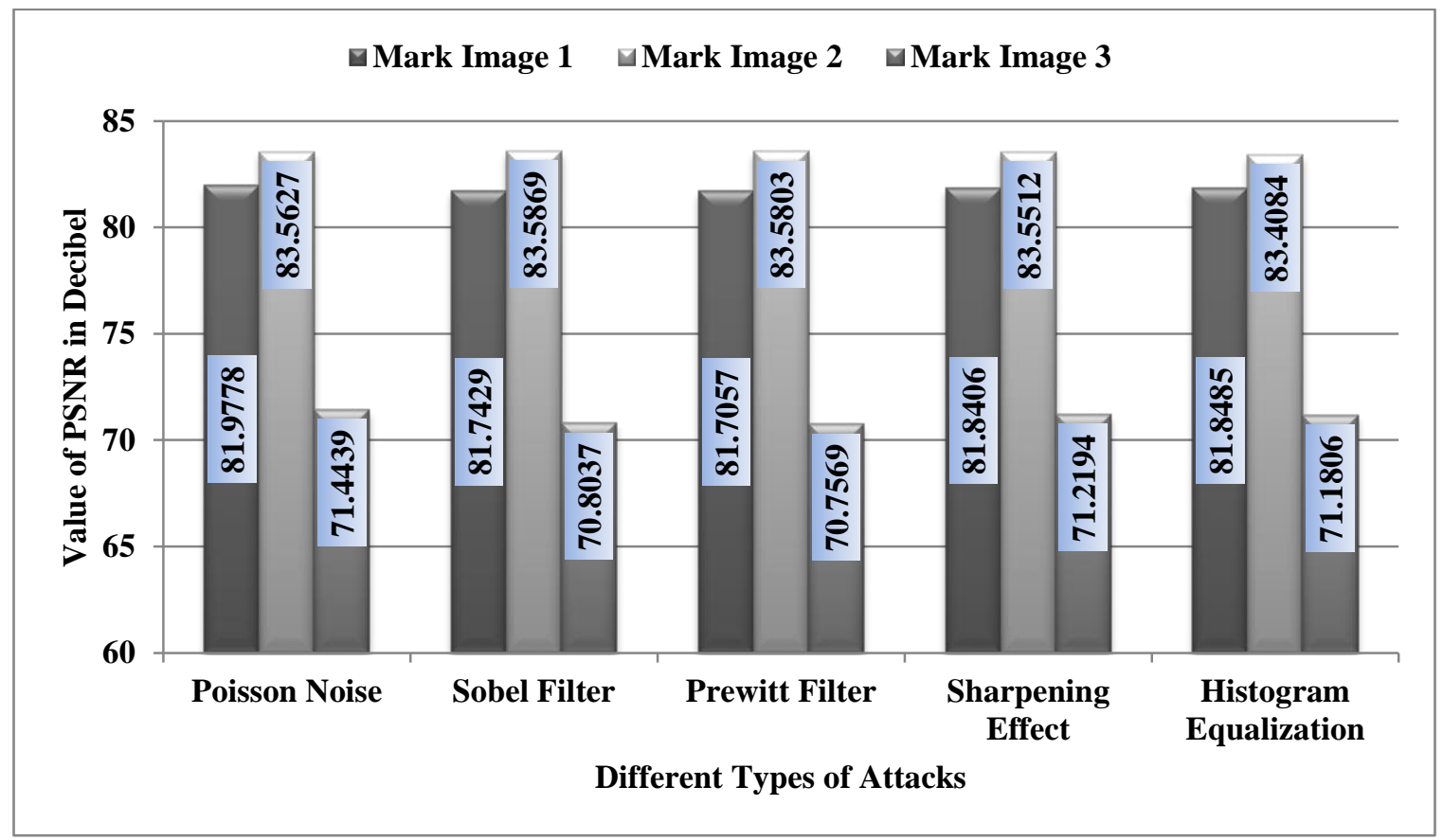

Fig. 1.19: PSNR of Mark Images Obtained After Being Extracted from the Host Image against Several Attacks. 


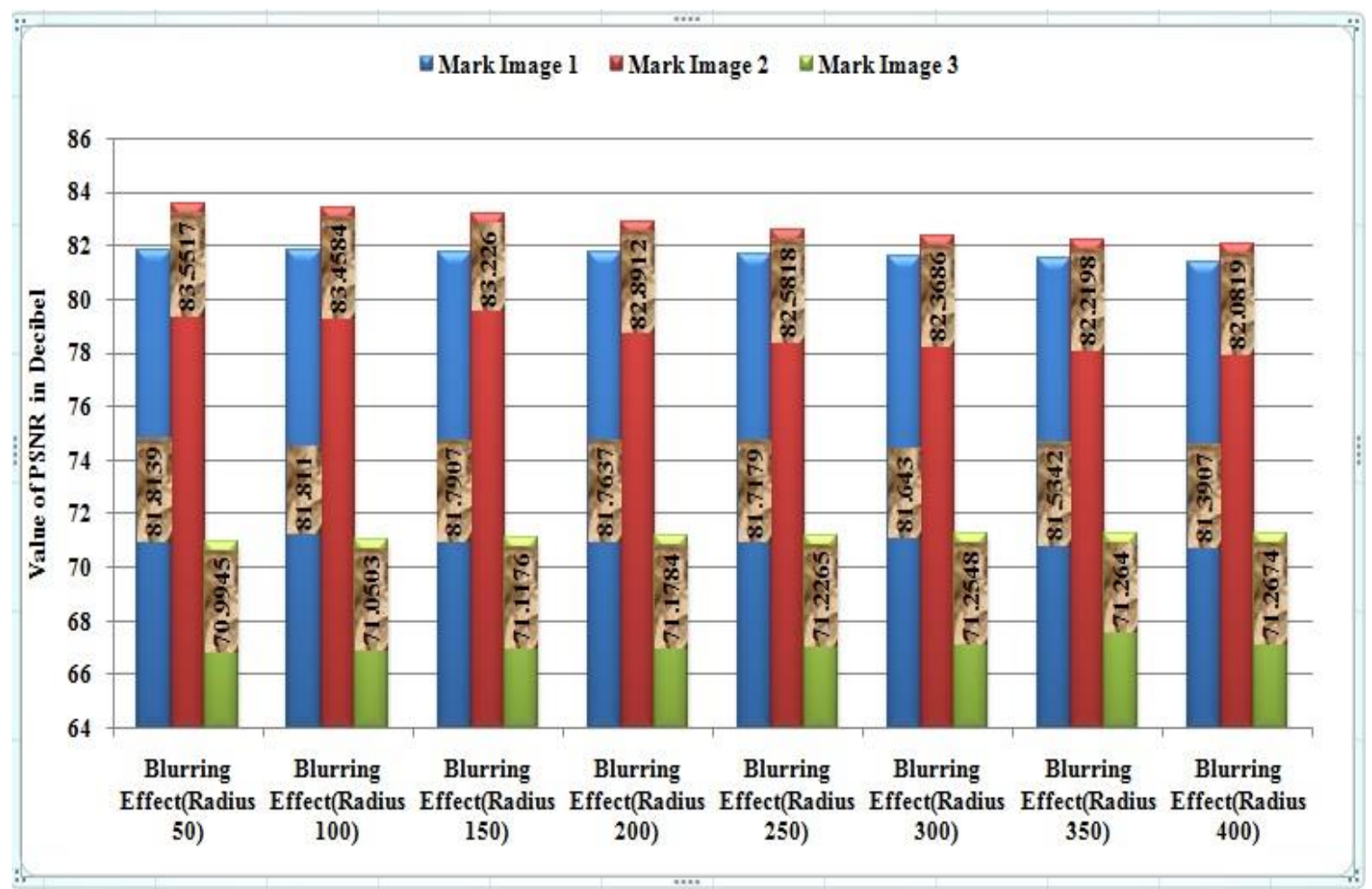

Fig. 1.20: PSNR of Mark Images Obtained After Being Extracted from the Host Image against 'Blurring Effect'.

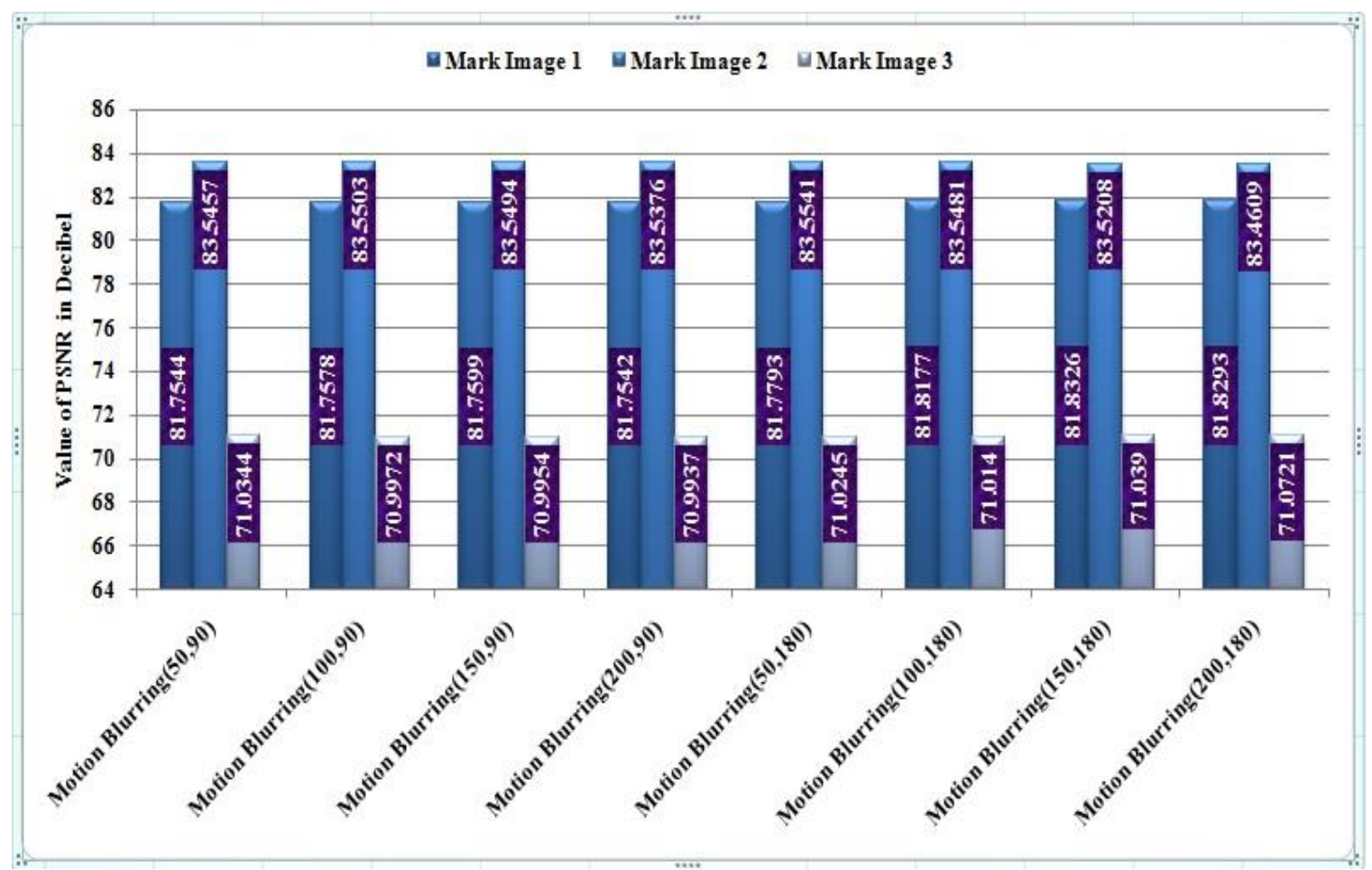

Fig. 1.21: PSNR of Mark Images Obtained After Being Extracted from the Host Image against 'Motion Blurring Effect'. 


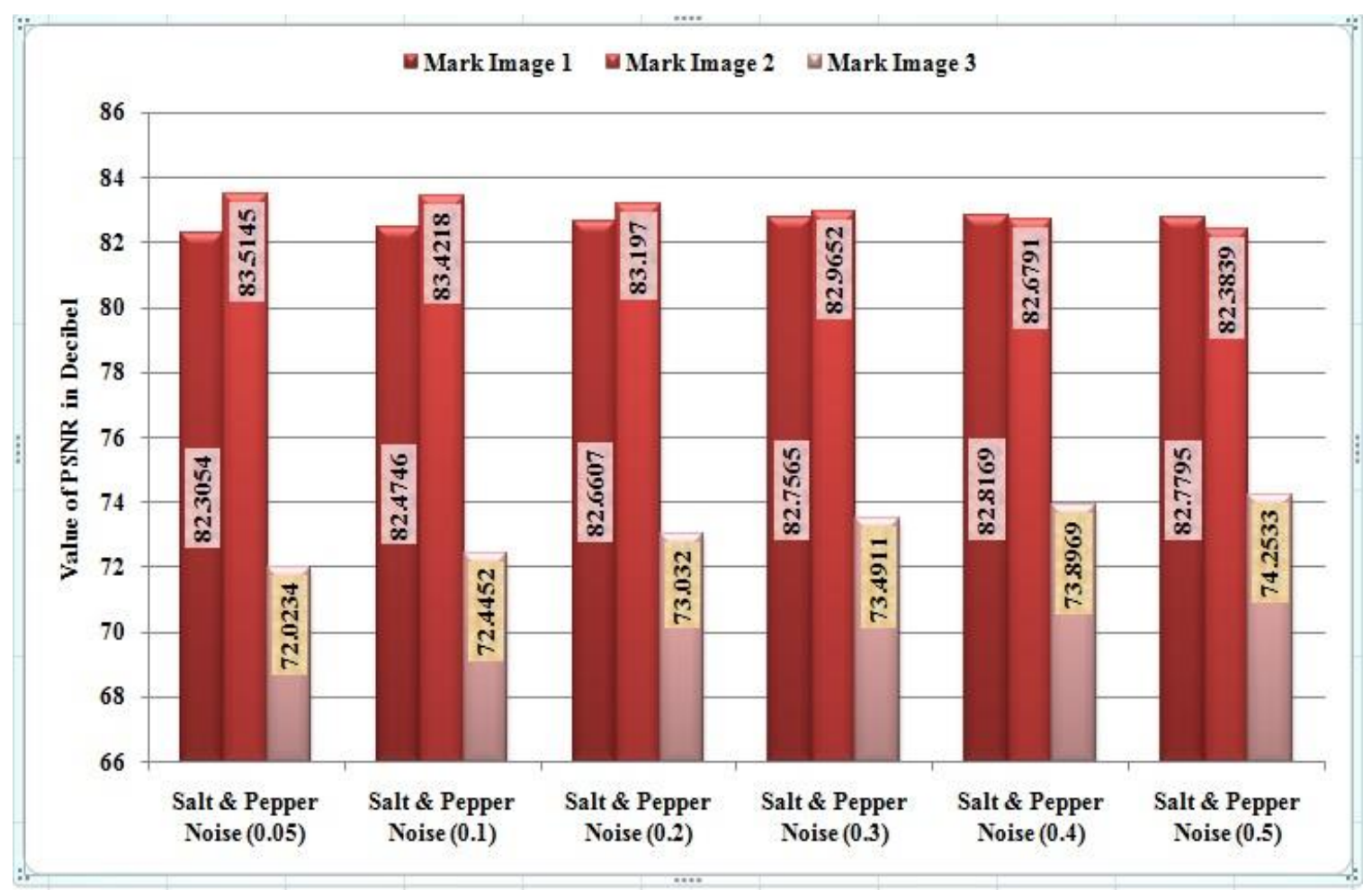

Fig. 1.22: PSNR of Mark Images Obtained After Being Extracted from the Host Image against 'Salt \& Pepper Noise'.

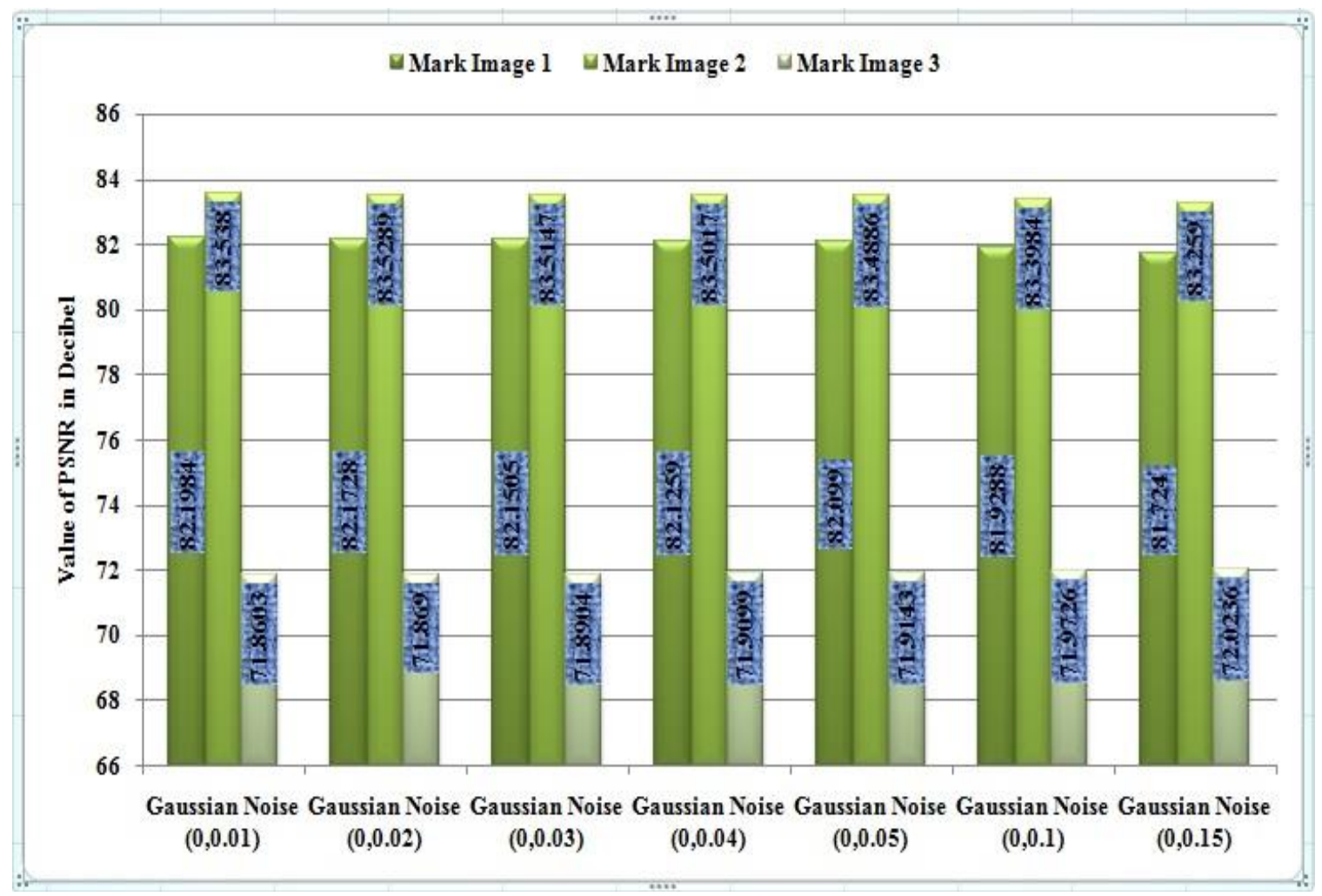

Fig. 1.23: PSNR of Mark Images Obtained After Being Extracted from the Host Image against 'Gaussian Noise'. 


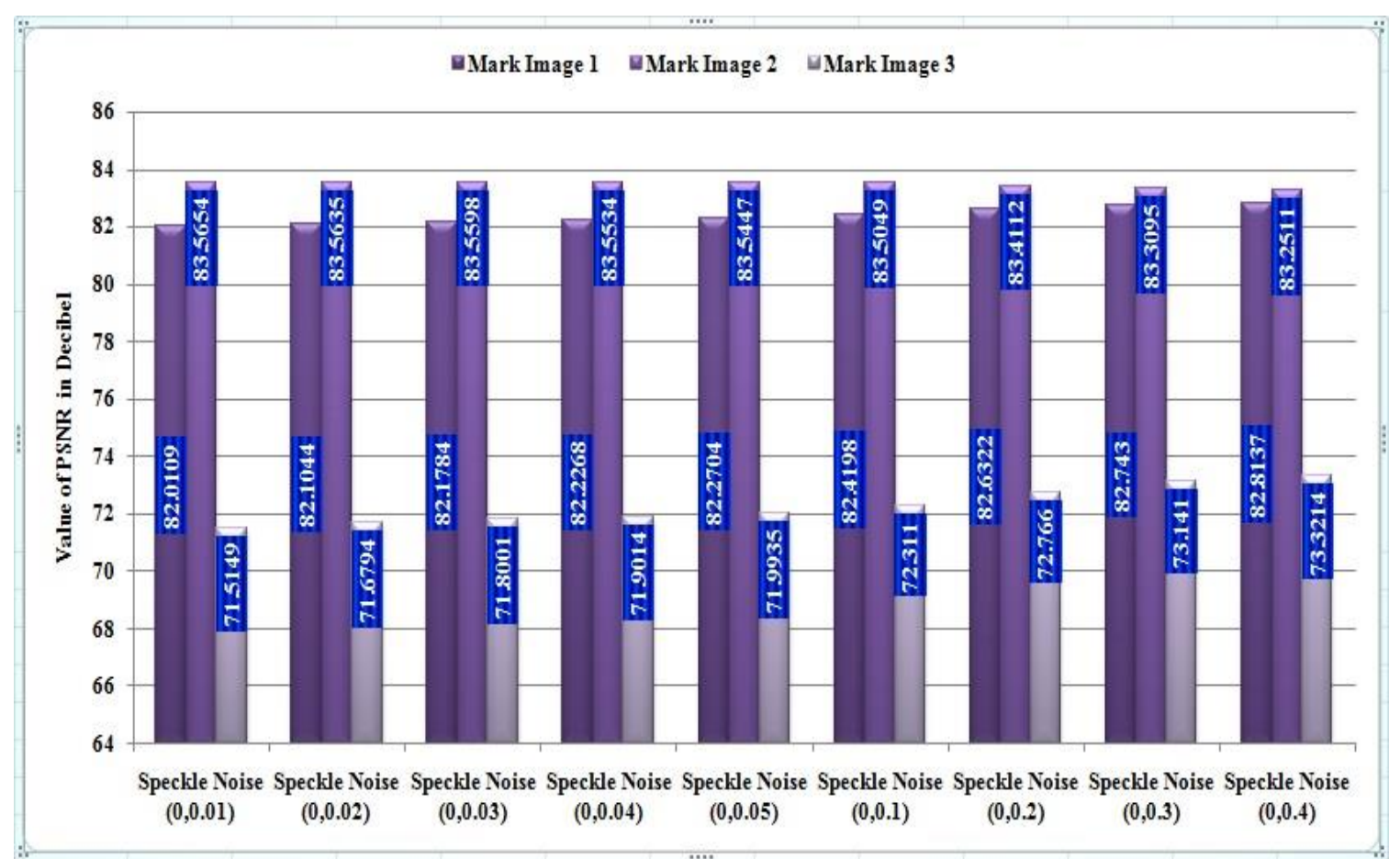

Fig. 1.24: PSNR of Mark Images Obtained After Being Extracted from the Host Image against 'Speckle Noise'.

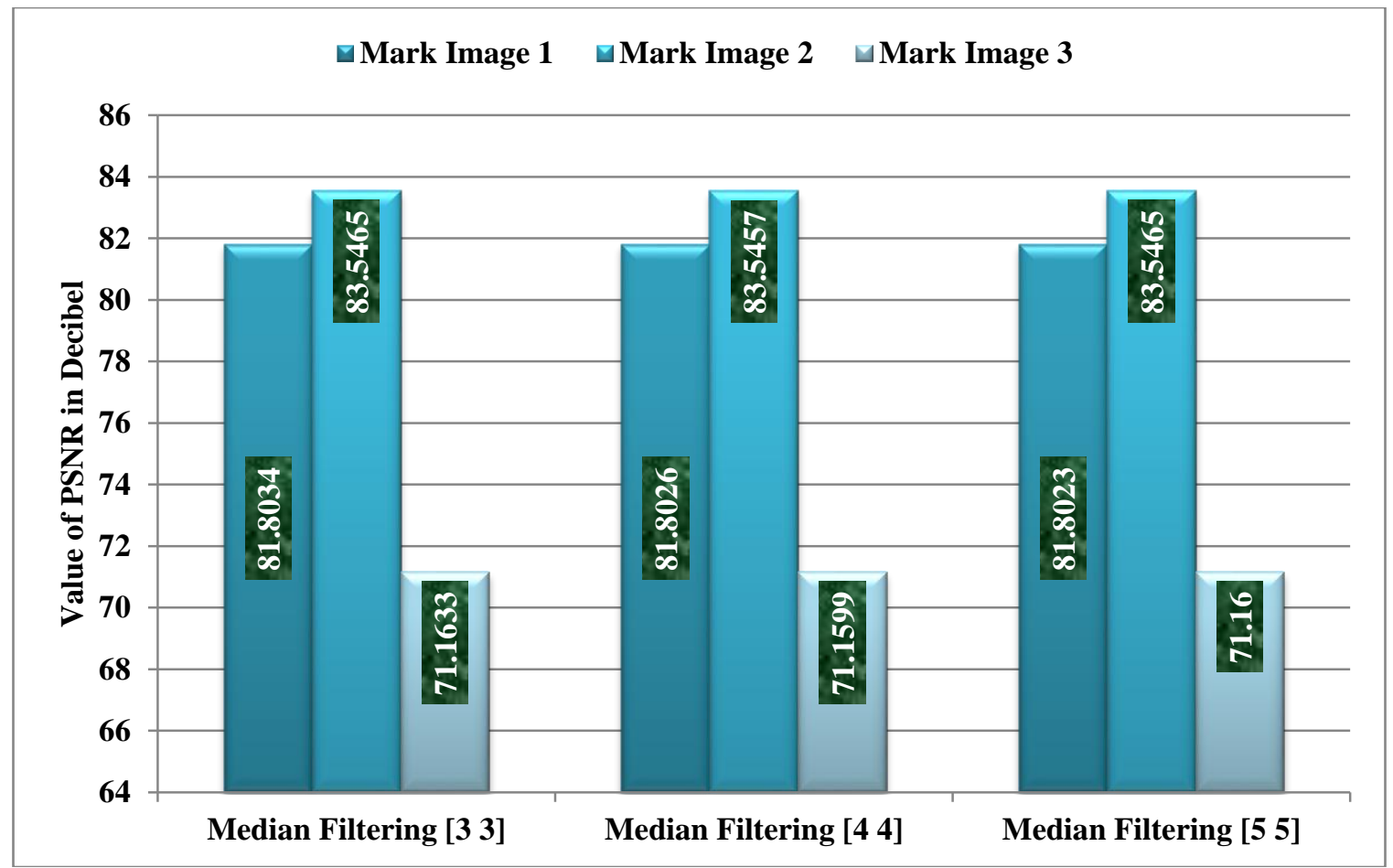

Fig. 1.25: PSNR of Mark Images Obtained After Being Extracted from the Host Image against 'Median Filtering'. 


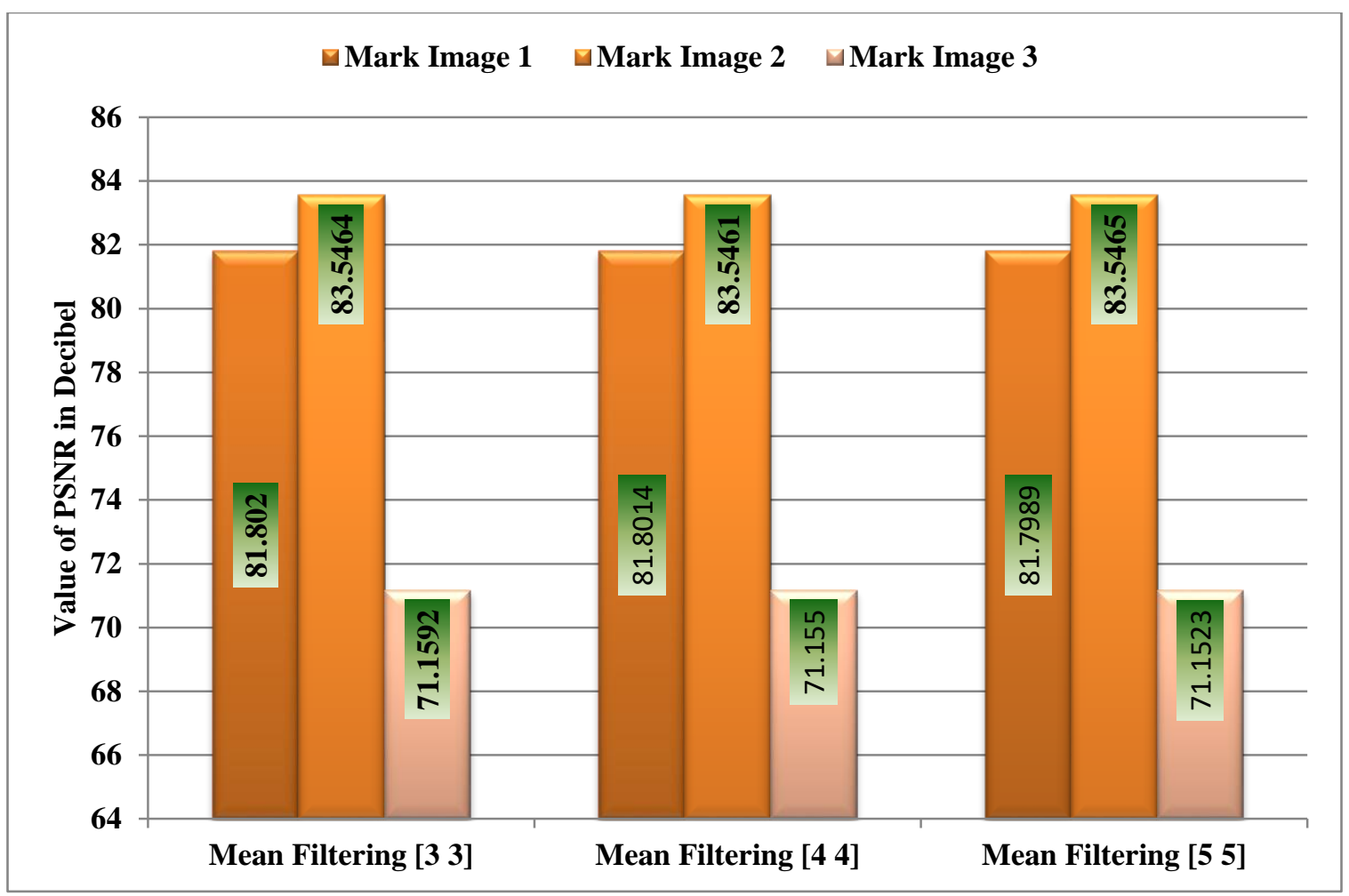

Fig. 1.26: PSNR of Mark Images Obtained After Being Extracted from the Host Image against 'Mean Filtering'.

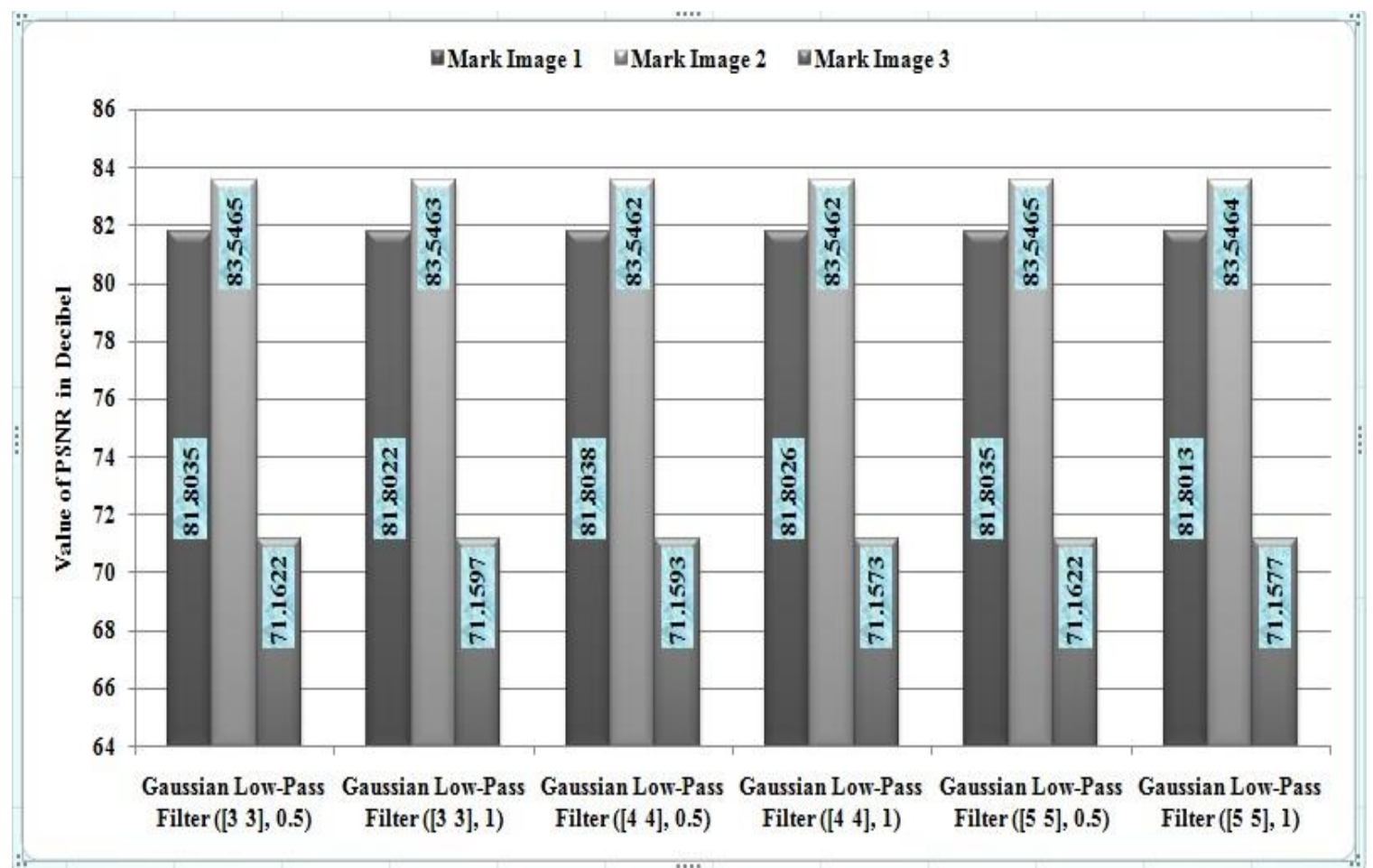

Fig. 1.27: PSNR of Mark Images Obtained After Being Extracted from the Host Image against 'Gaussian Low-Pass Filter'. 


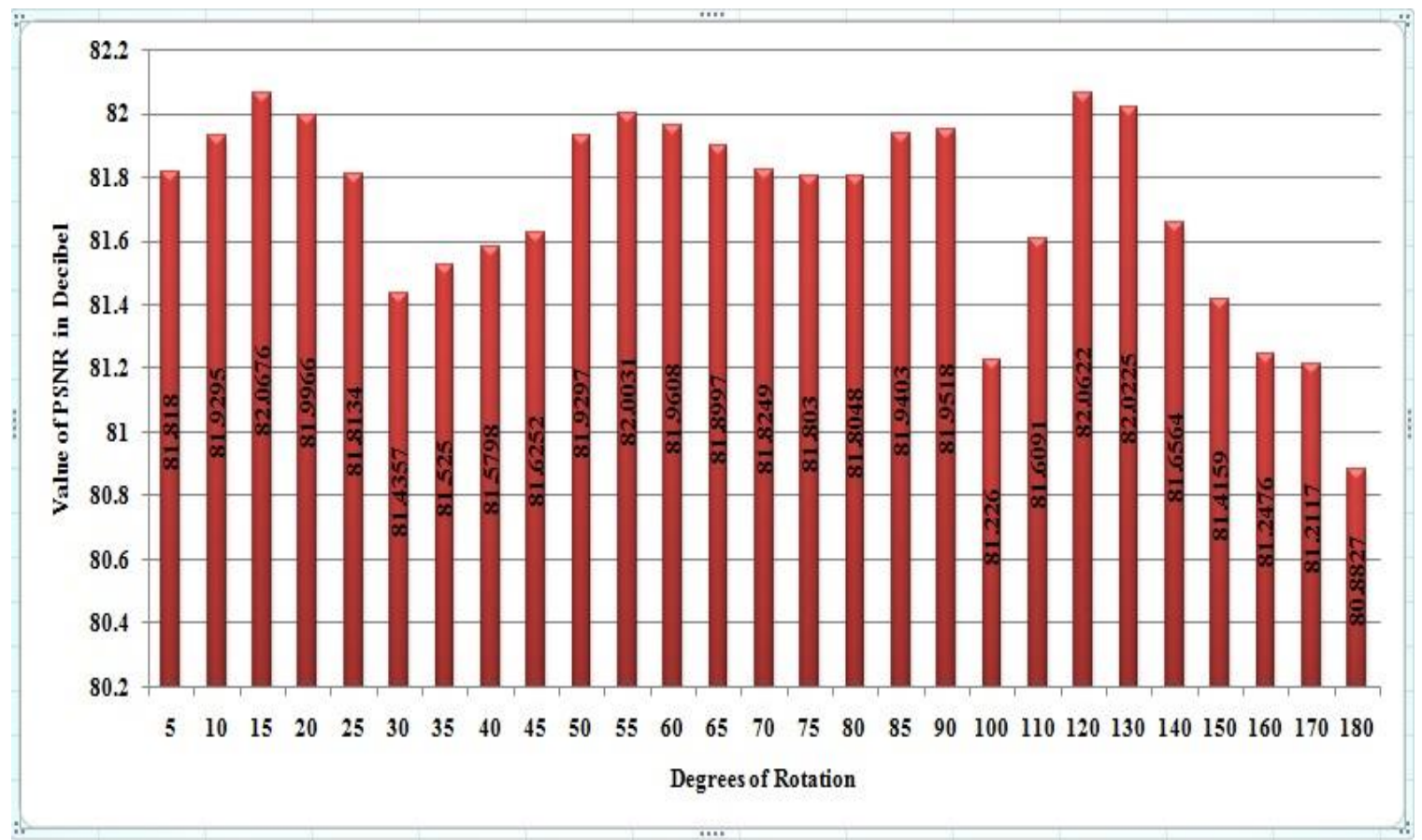

Fig. 1.28: PSNR of Mark Image1 Obtained After Being Extracted from the Host Image against 'Rotation (Anti-Clockwise)'.

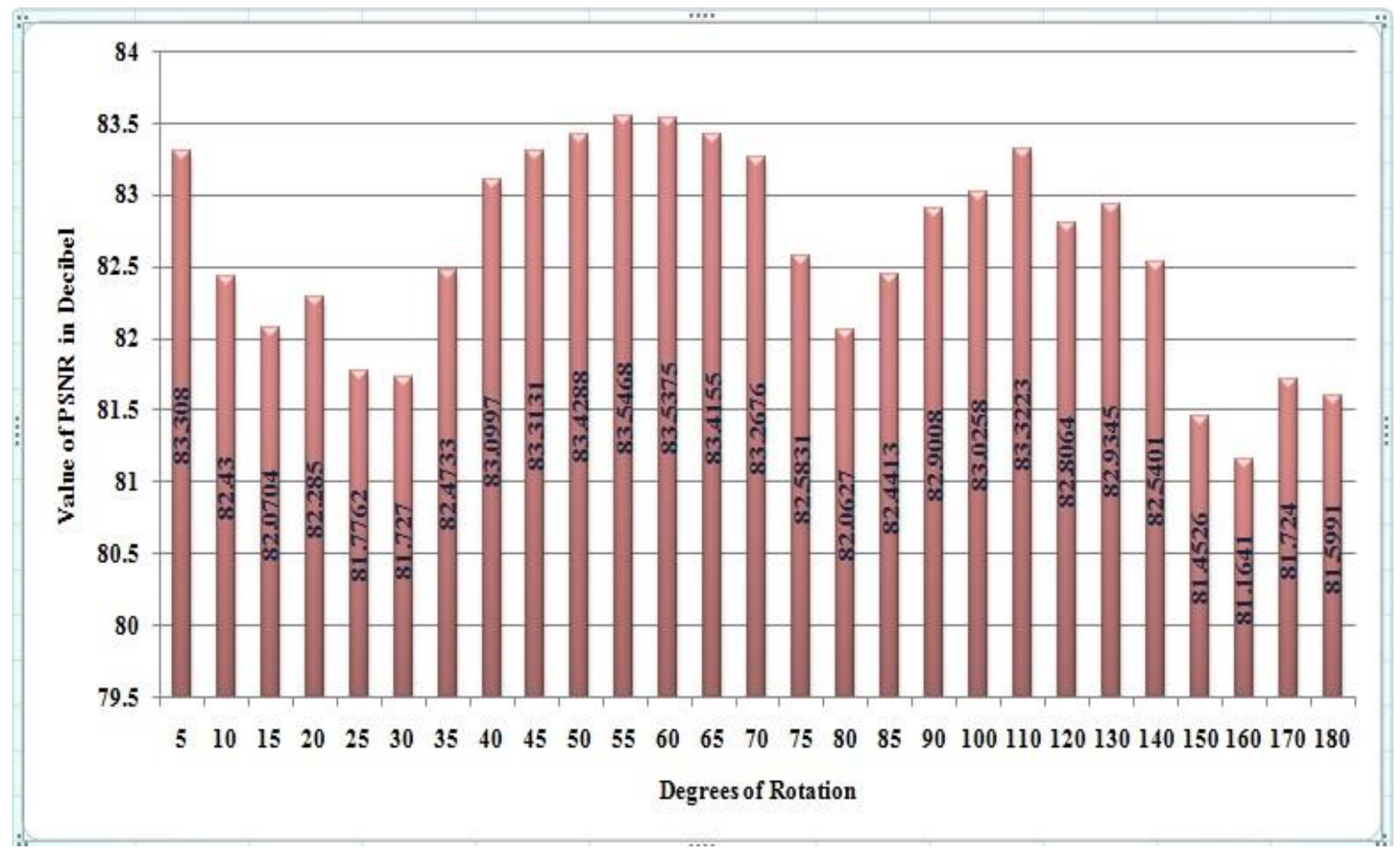

Fig. 1.29: PSNR of Mark Image2 Obtained After Being Extracted from the Host Image against 'Rotation (Anti-Clockwise)'. 


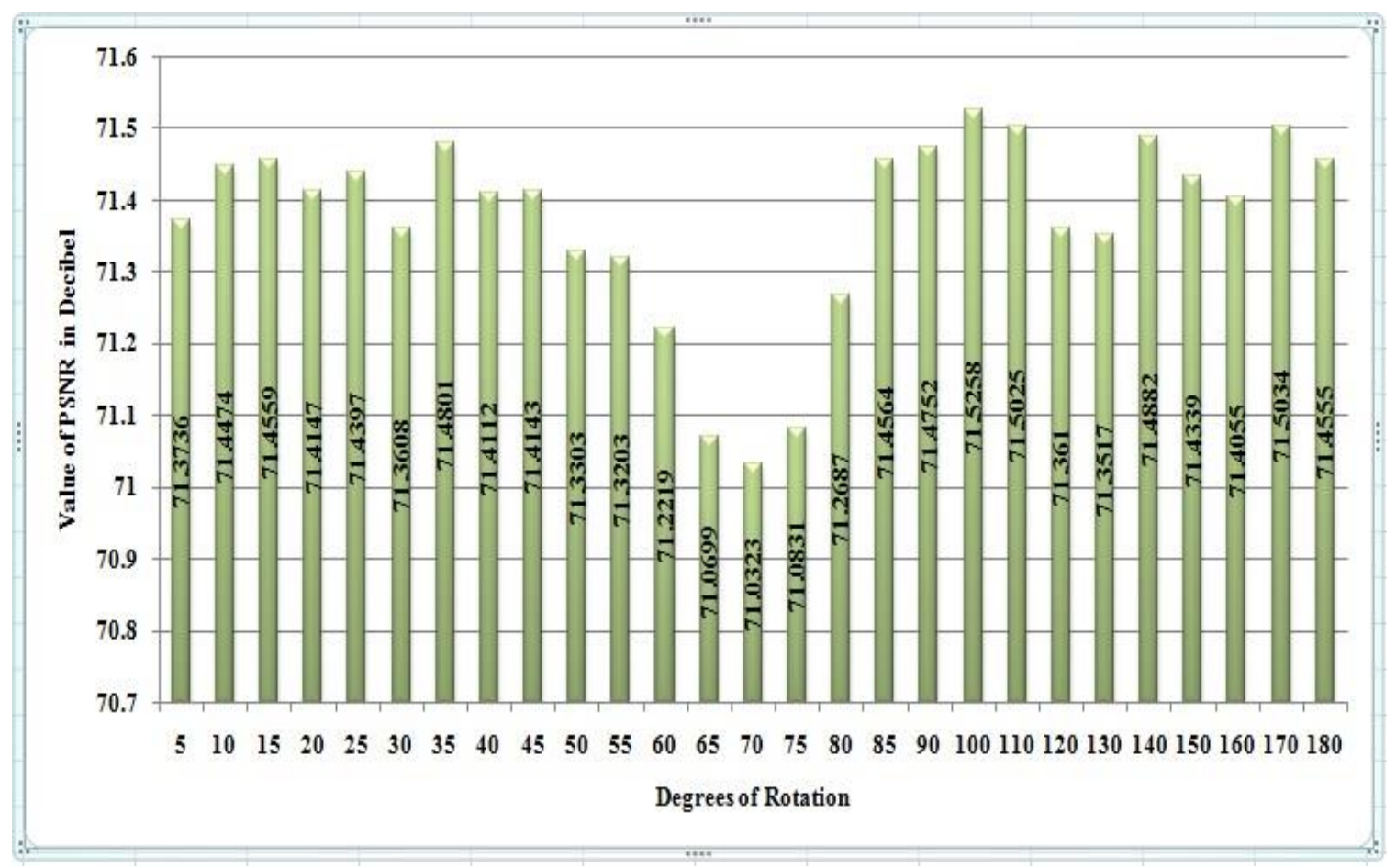

Fig. 1.30: PSNR of Mark Image3 Obtained After Being Extracted from the Host Image against 'Rotation (Anti-Clockwise)'.

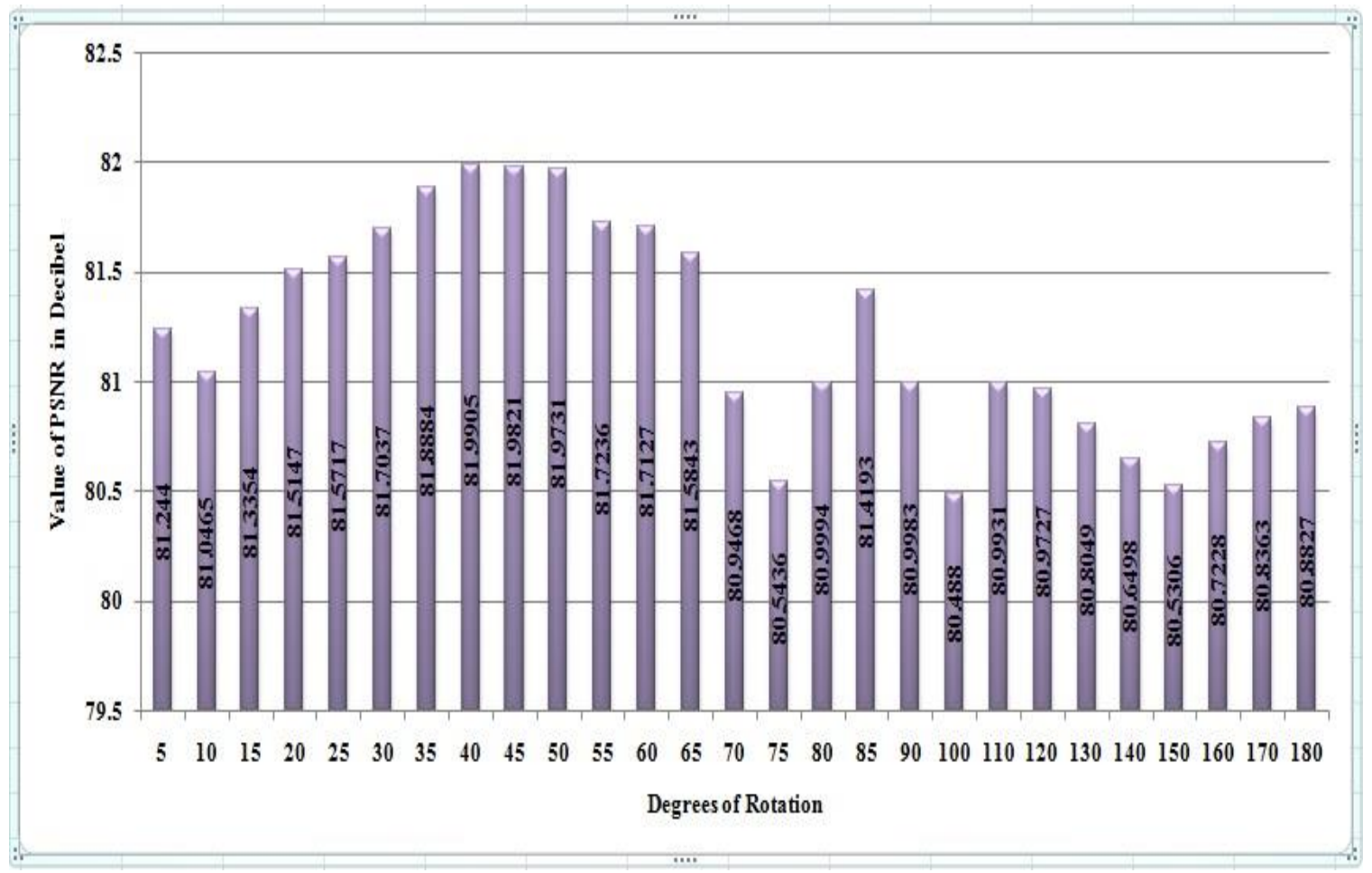

Fig. 1.31: PSNR of Mark Image1 Obtained After Being Extracted from the Host Image against 'Rotation (Clockwise)'. 


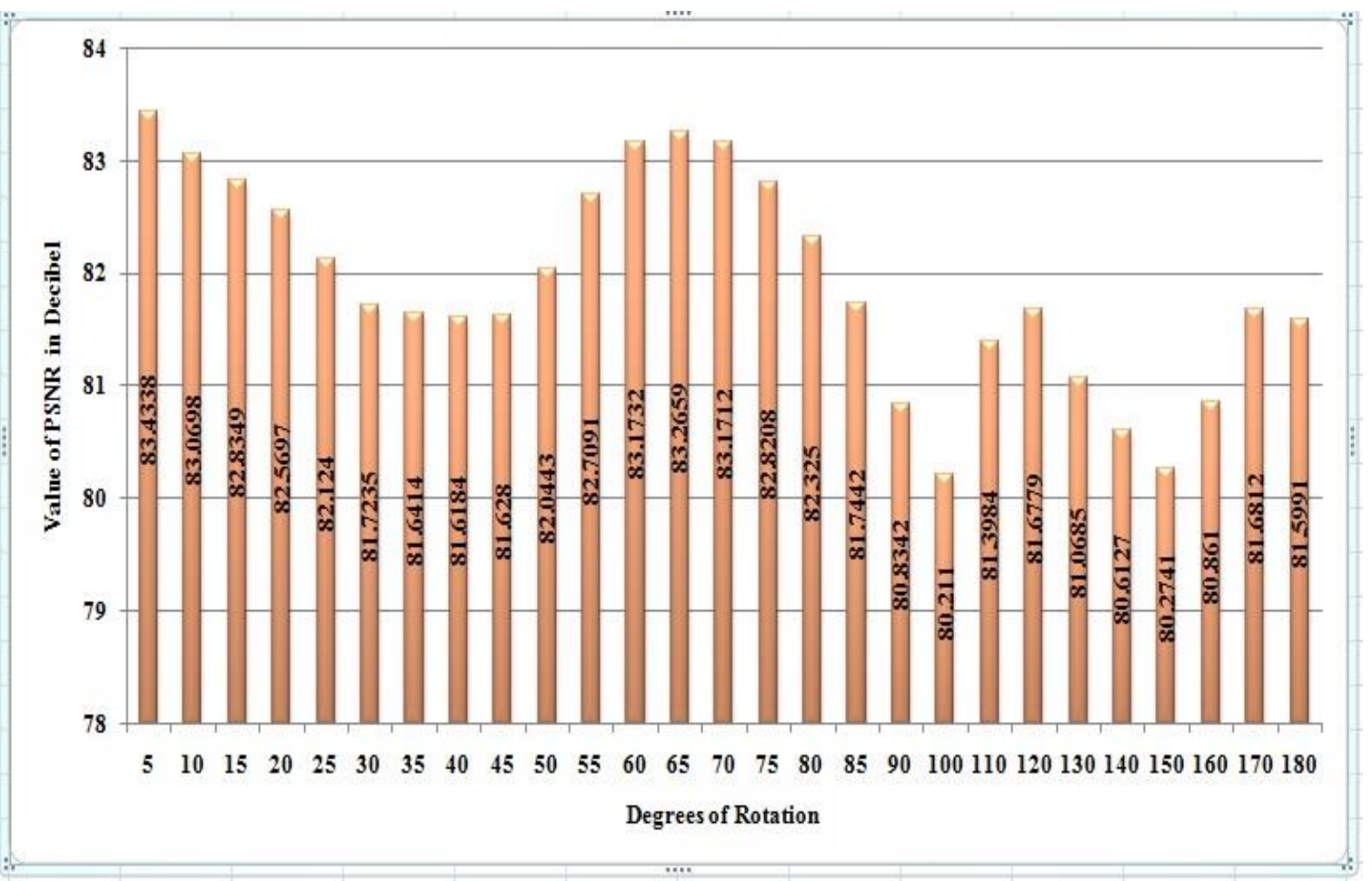

Fig. 1.32: PSNR of Mark Image2 Obtained After Being Extracted from the Host Image against 'Rotation (Clockwise)'.

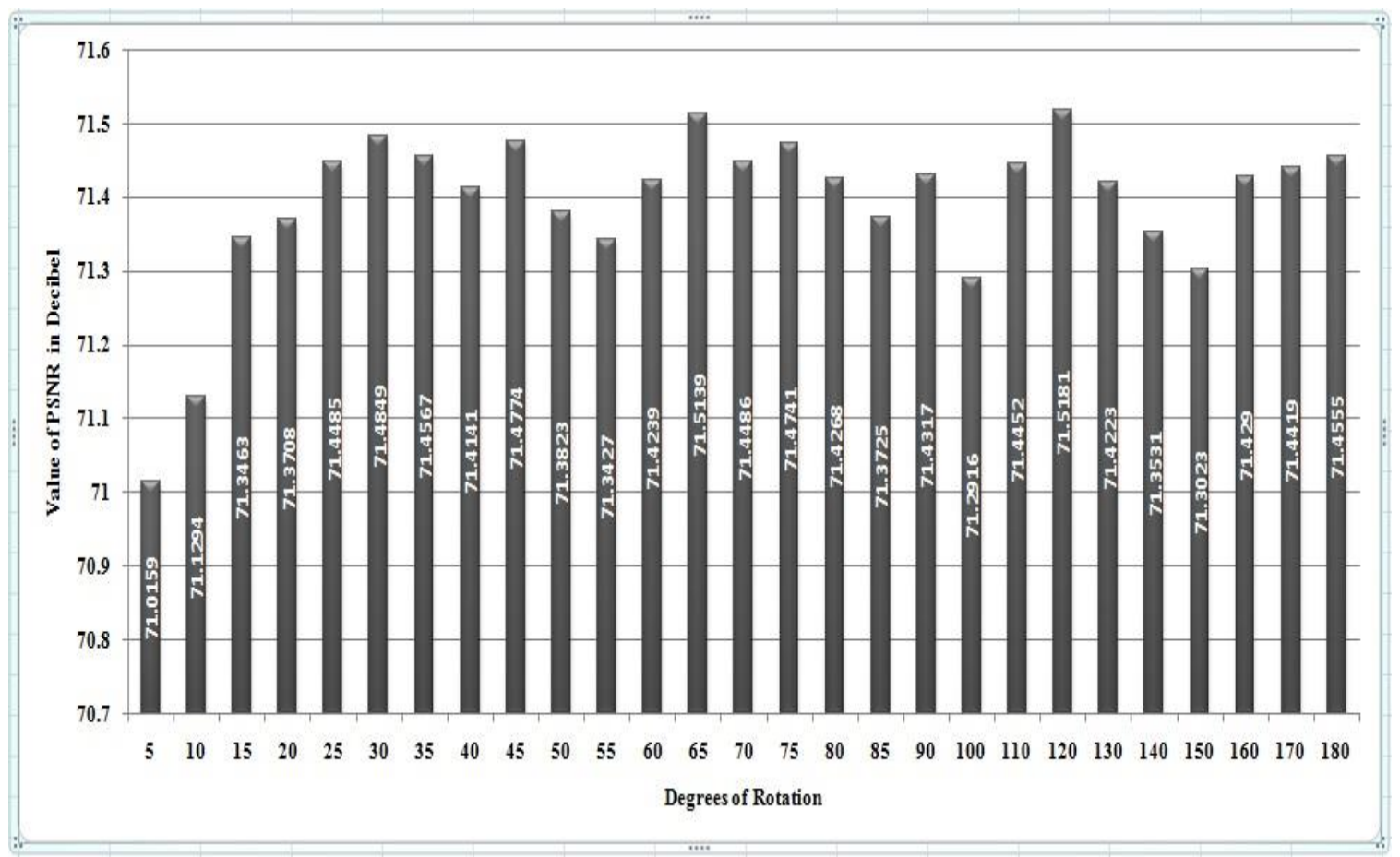

Fig. 1.33: PSNR of Mark Image3 Obtained After Being Extracted from the Host Image against 'Rotation (Clockwise)'. 
From figures 1.14-1.33, it is seen that the proposed methodology work well under the effect of several attacks and filtering. The higher value of PSNR of the mark images and the values of NC as well as NCC over 0.9 are the proofs of the good performance of the proposed methodology. From those figures one point should be noted that the PSNR value of the third watermark is low in comparison with the values of PSNR of the other two mark images. The probable reason is that, the third watermark is embedded into the HH sub-band which contains the high frequency part of a signal and it is known that any kind of noise has high frequency. So, it can be said that the HH sub-band acts as the noisy part of a signal as that band contains the minimum portion of energy of a signal. So embedding a mark image into that band will certainly have a comparatively higher effect on the energy distribution of that mark image than those of the other two mark images. Because of this reason the value of PSNR of the third watermark image is comparatively low. Figures 1.34-1.49 pictorially represent the successful extraction of the mark images after applying several attacks and filtering action on the host image. So, it can be said that the proposed watermarking method is robust. 


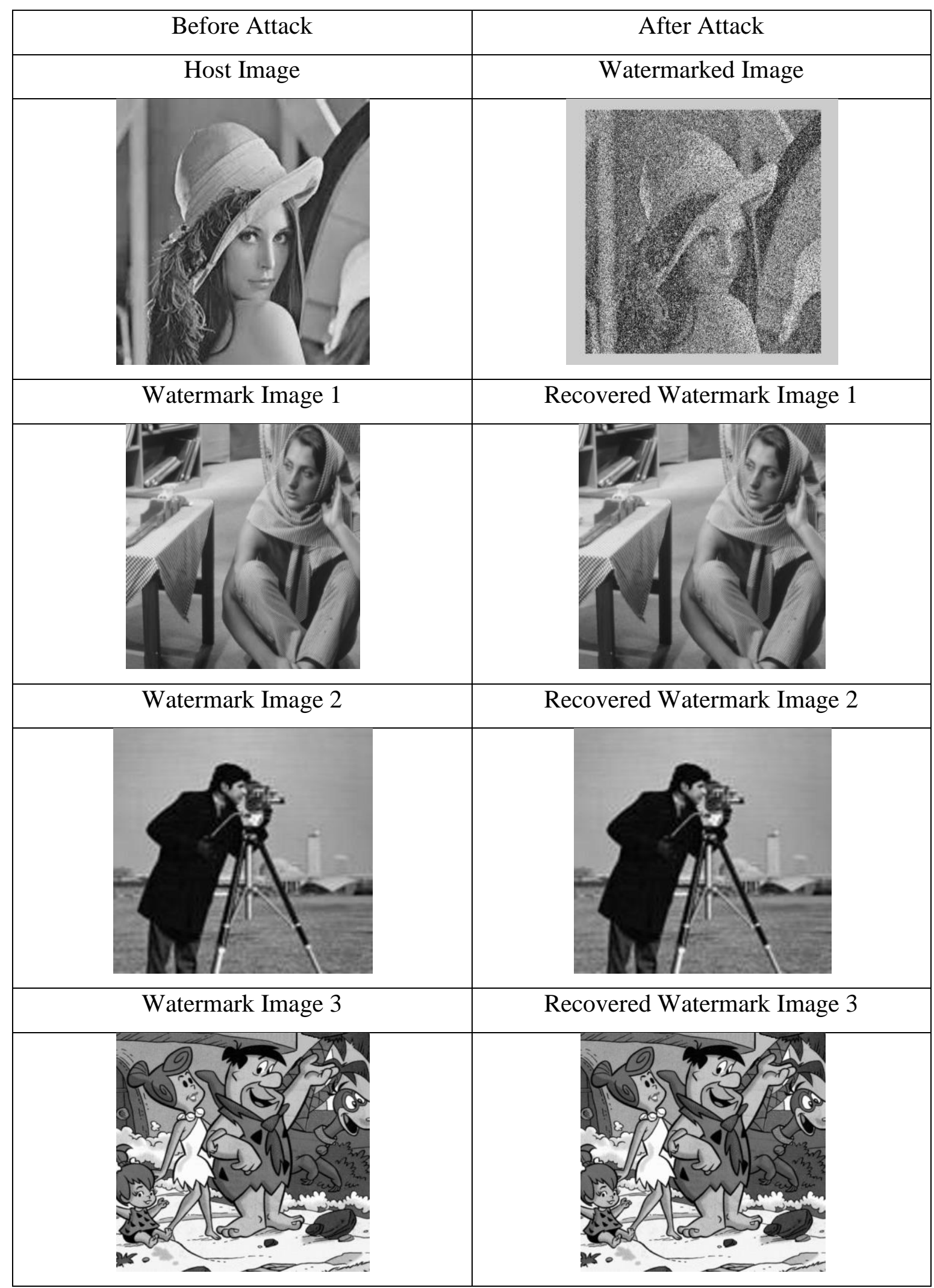

Fig.1.34: Extraction of Watermark Images against 'Salt \& Pepper Noise'. 


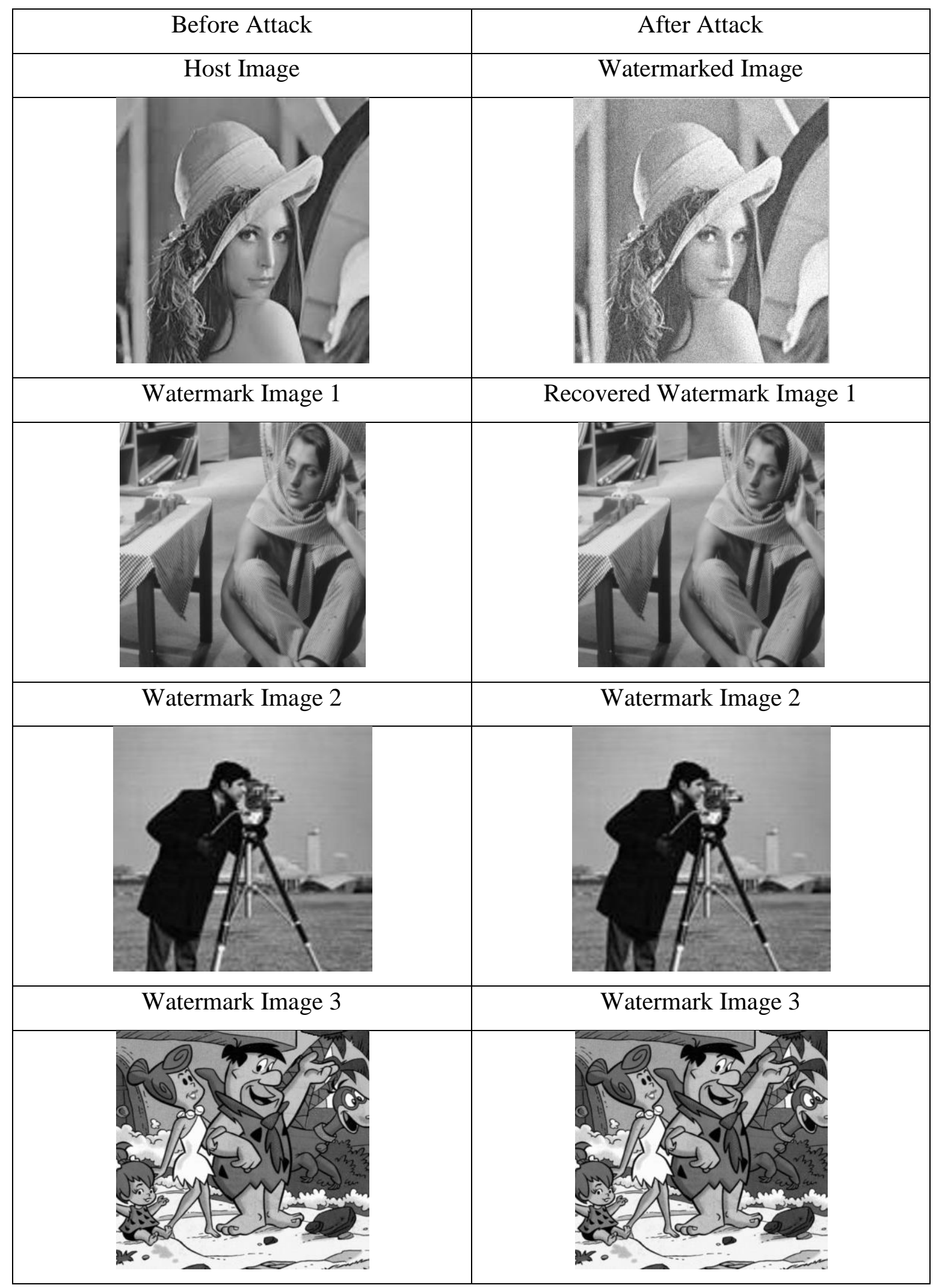

Fig.1.35: Extraction of Watermark Images against 'Gaussian Noise'. 


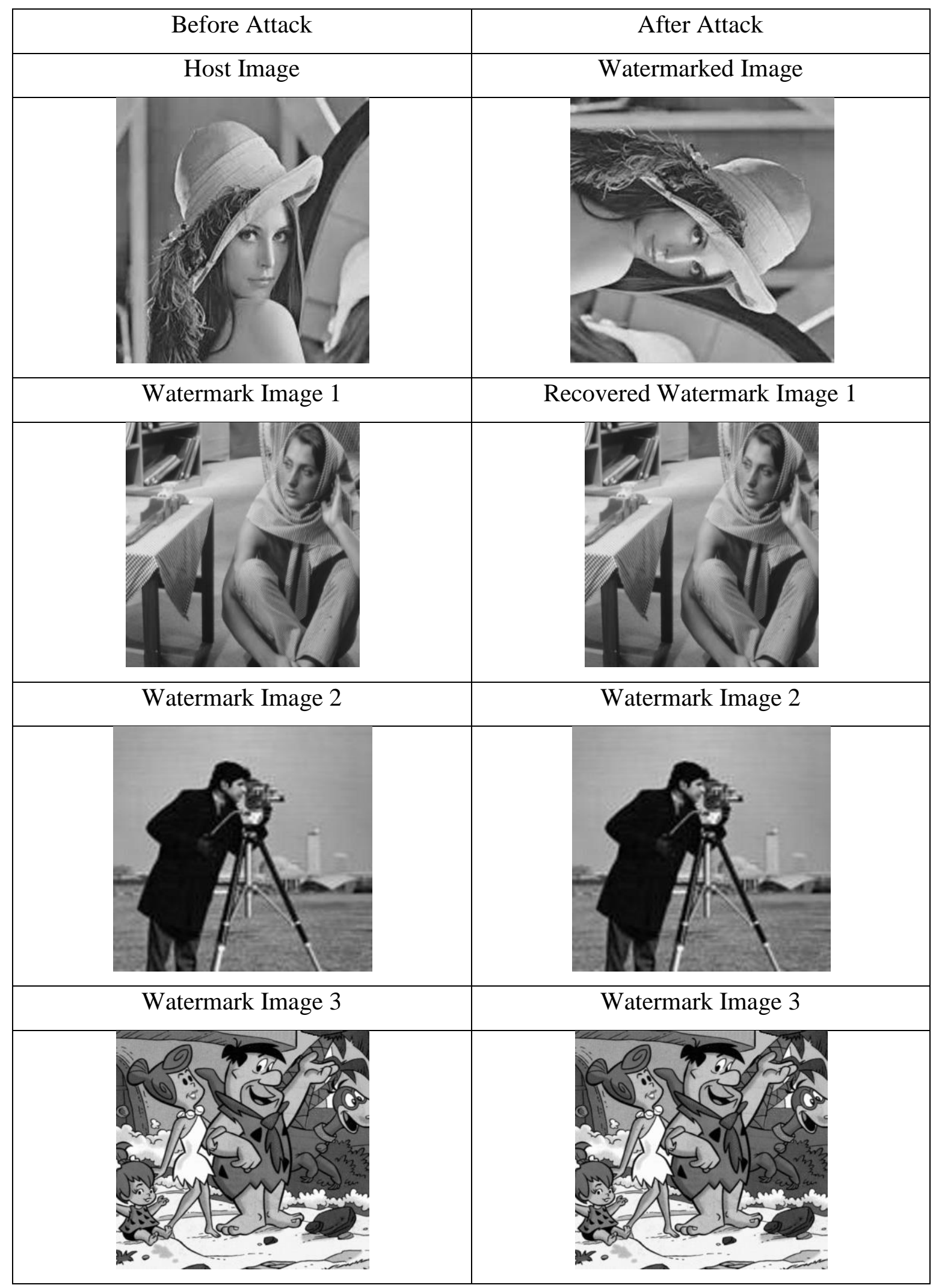

Fig.1.36: Extraction of Watermark Images against 'Rotation (Clockwise)'. 


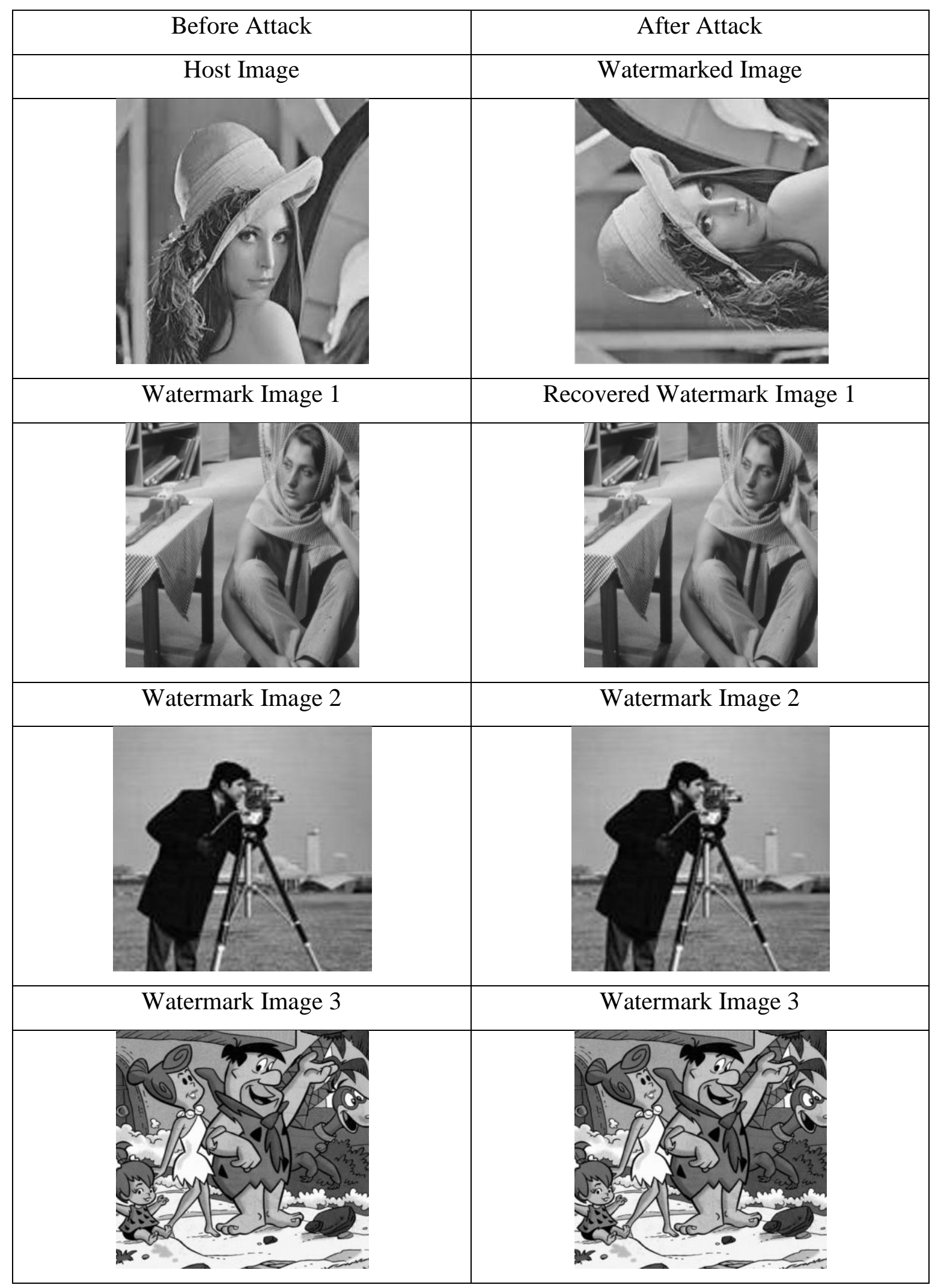

Fig.1.37: Extraction of Watermark Images against 'Rotation (Anti-Clockwise)'. 


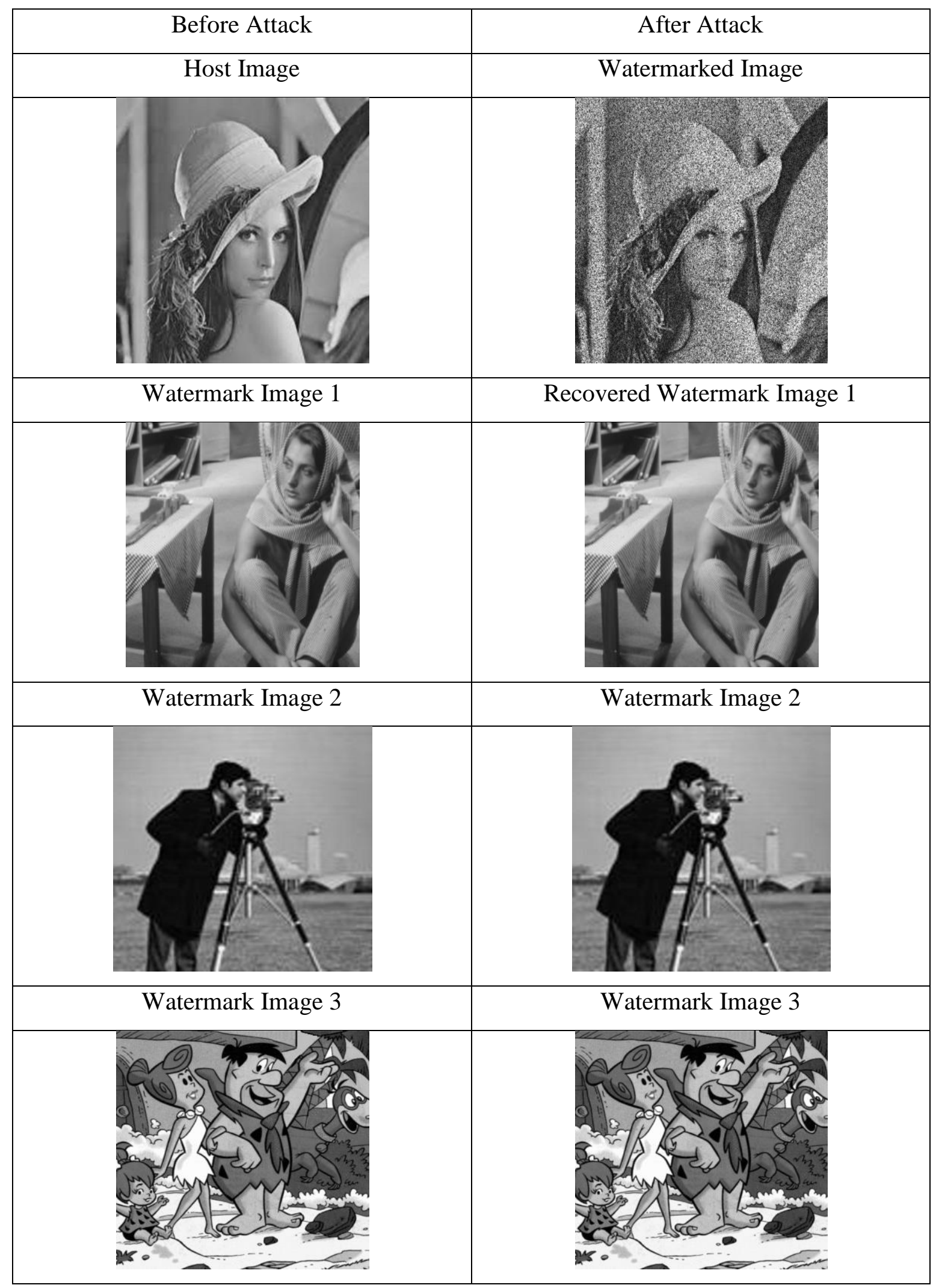

Fig.1.38: Extraction of Watermark Images against 'Speckle Noise'. 


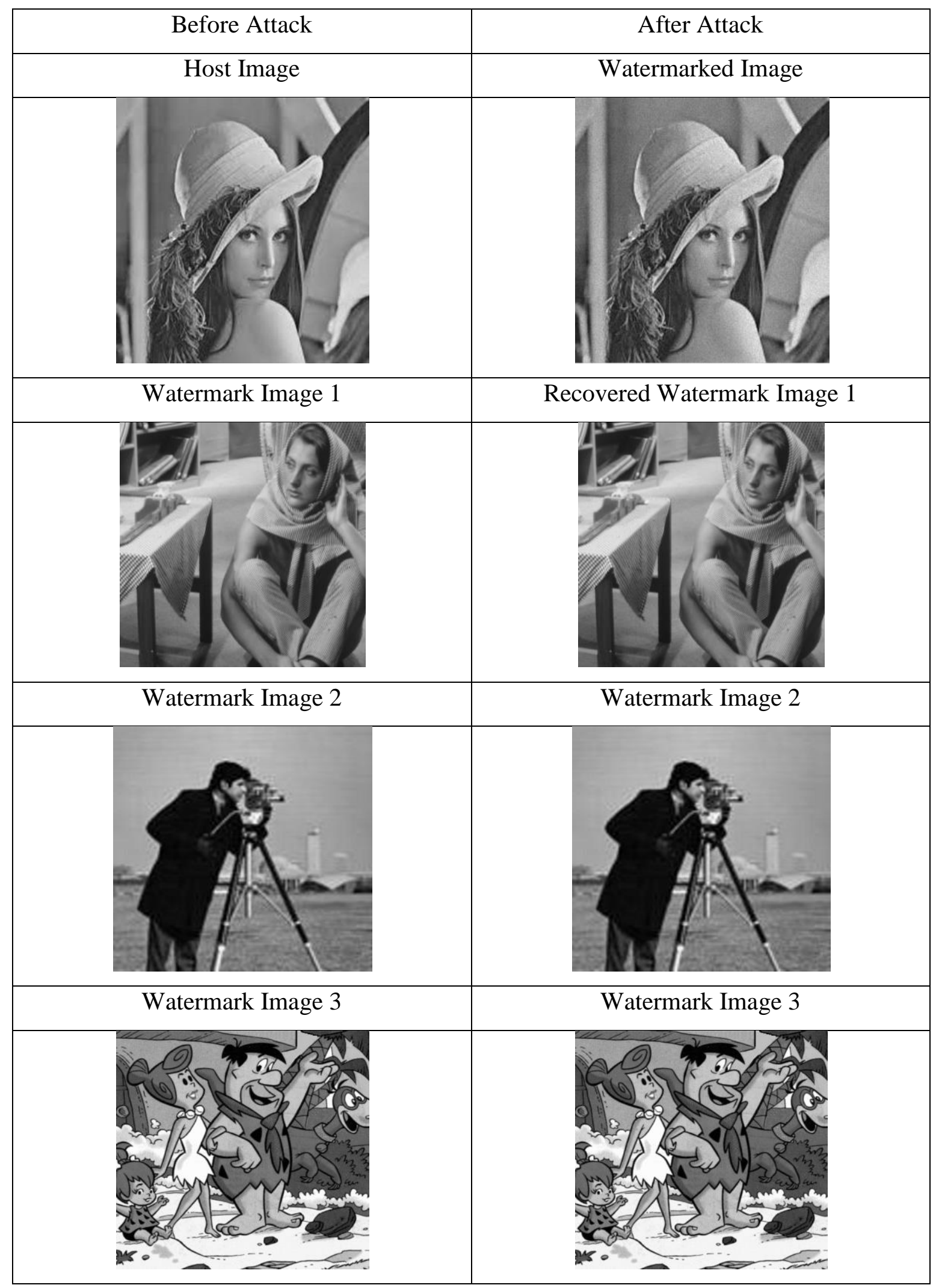

Fig.1.39: Extraction of Watermark Images against 'Poisson Noise'. 


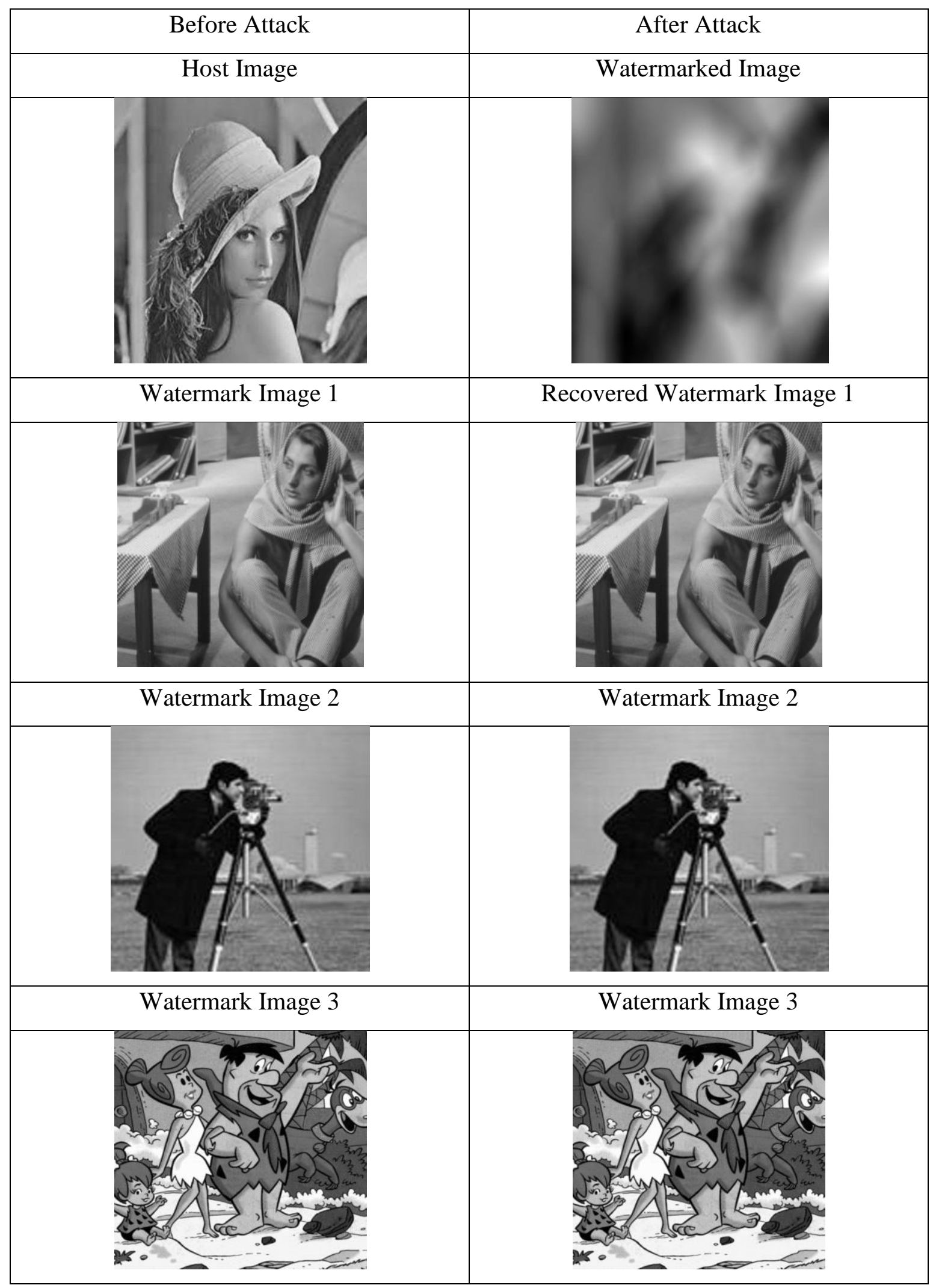

Fig.1.40: Extraction of Watermark Images against 'Blurring Effect' 


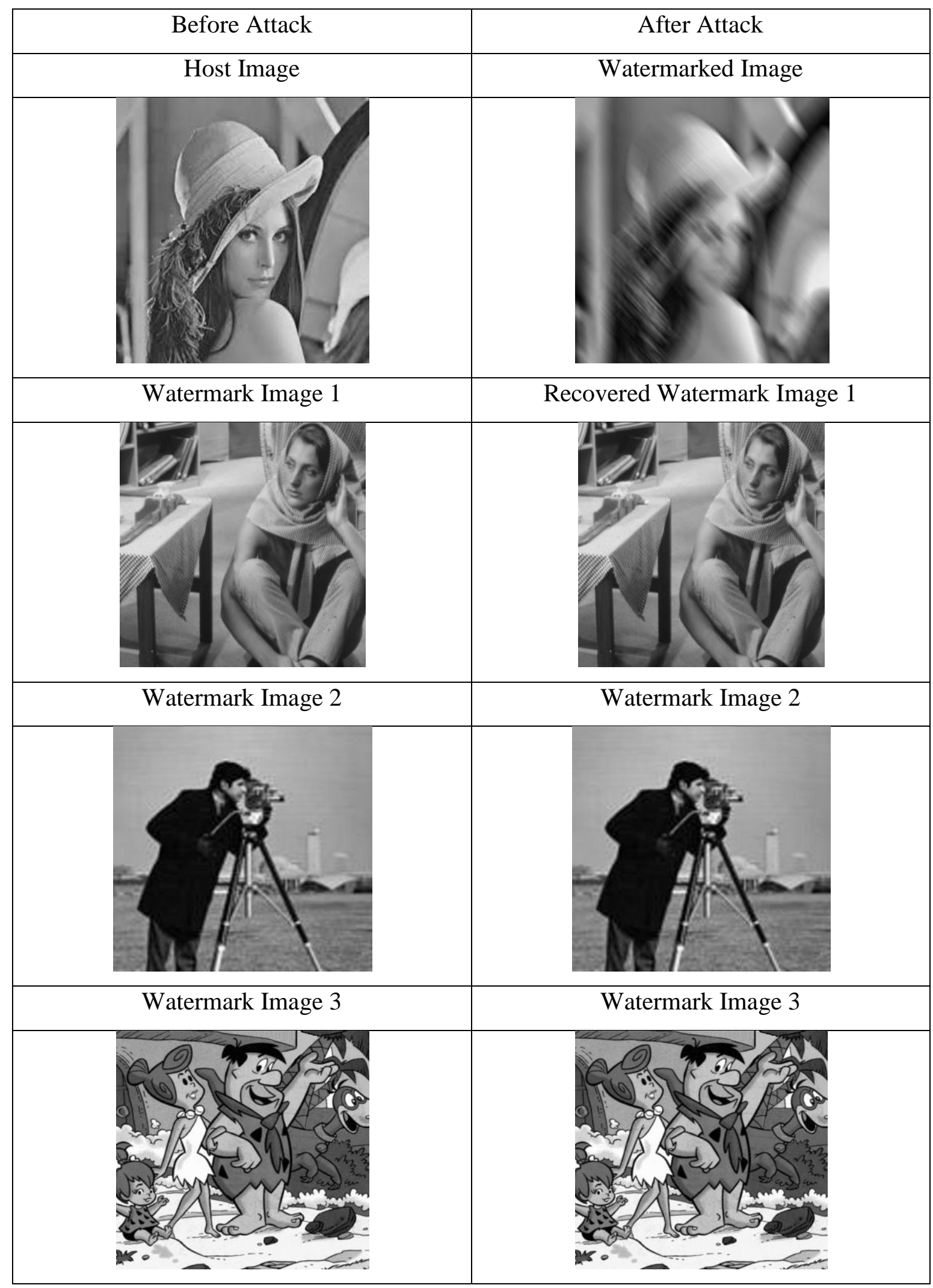

Fig.1.41: Extraction of Watermark Images against 'Motion Blurring Effect'. 


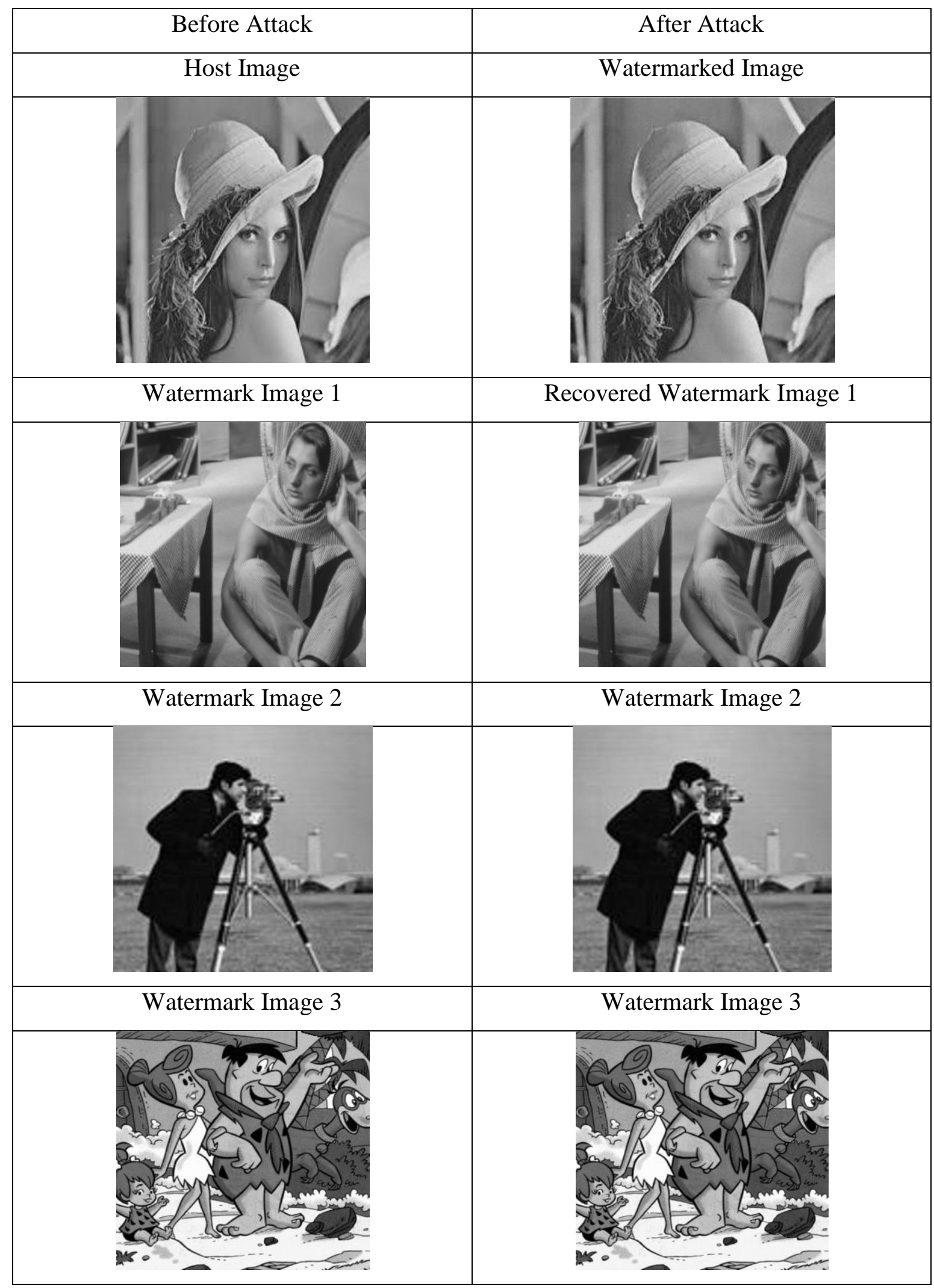

Fig.1.42: Extraction of Watermark Images against 'Sharpening Effect'. 


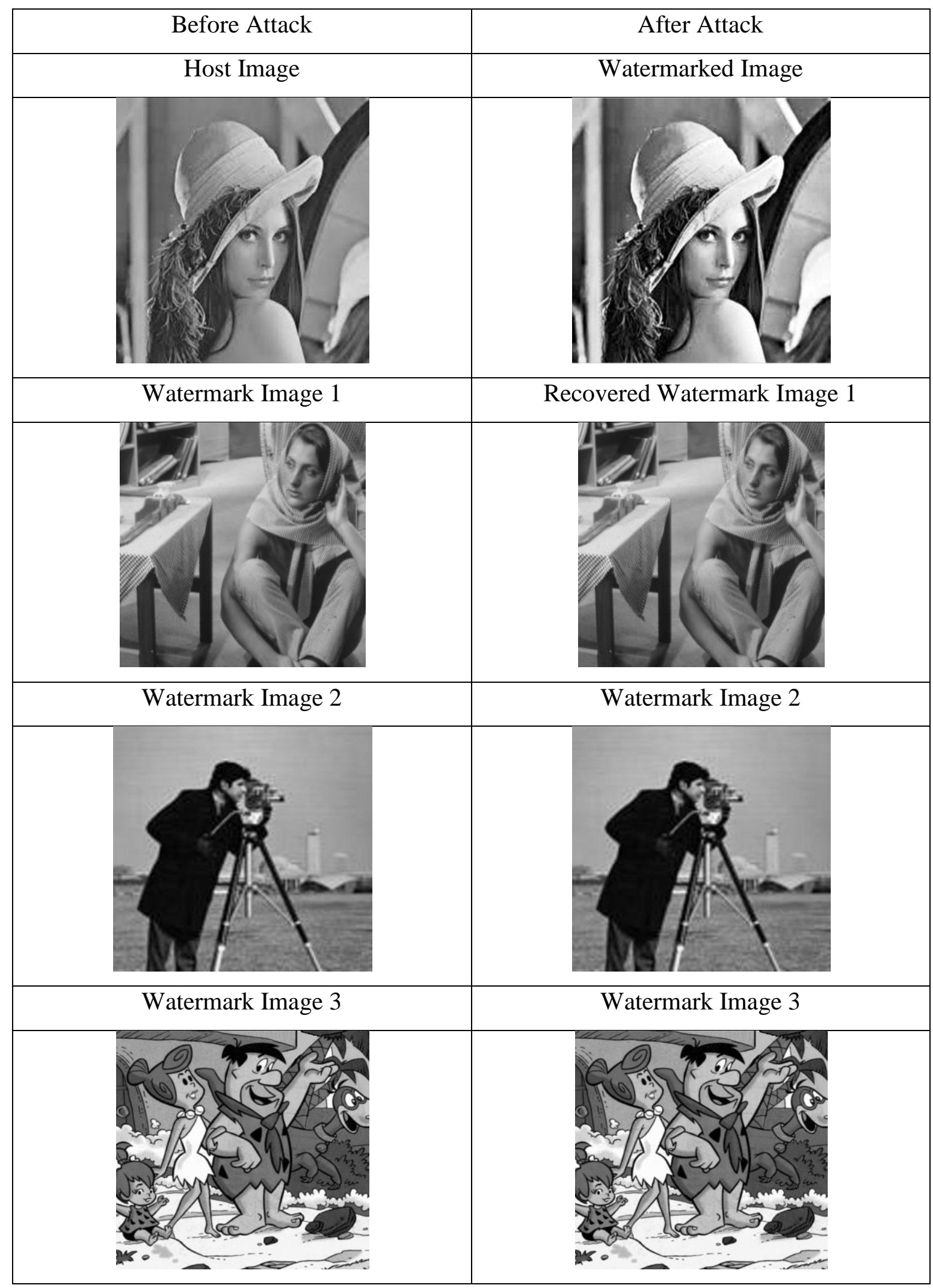

Fig.1.43: Extraction of Watermark Images against 'Histogram Equalization'. 


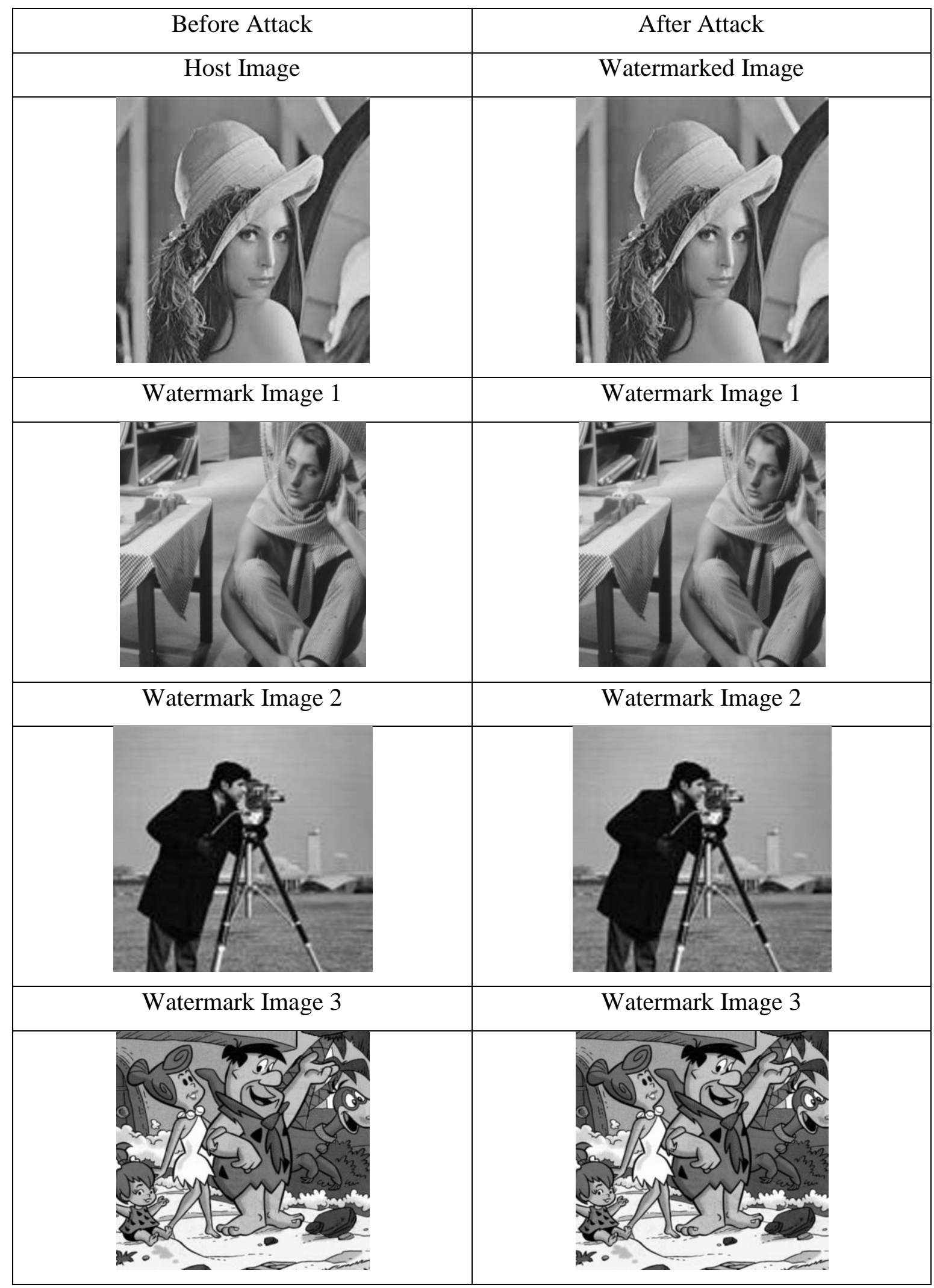

Fig.1.44: Extraction of Watermark Images against 'Median Filtering'. 


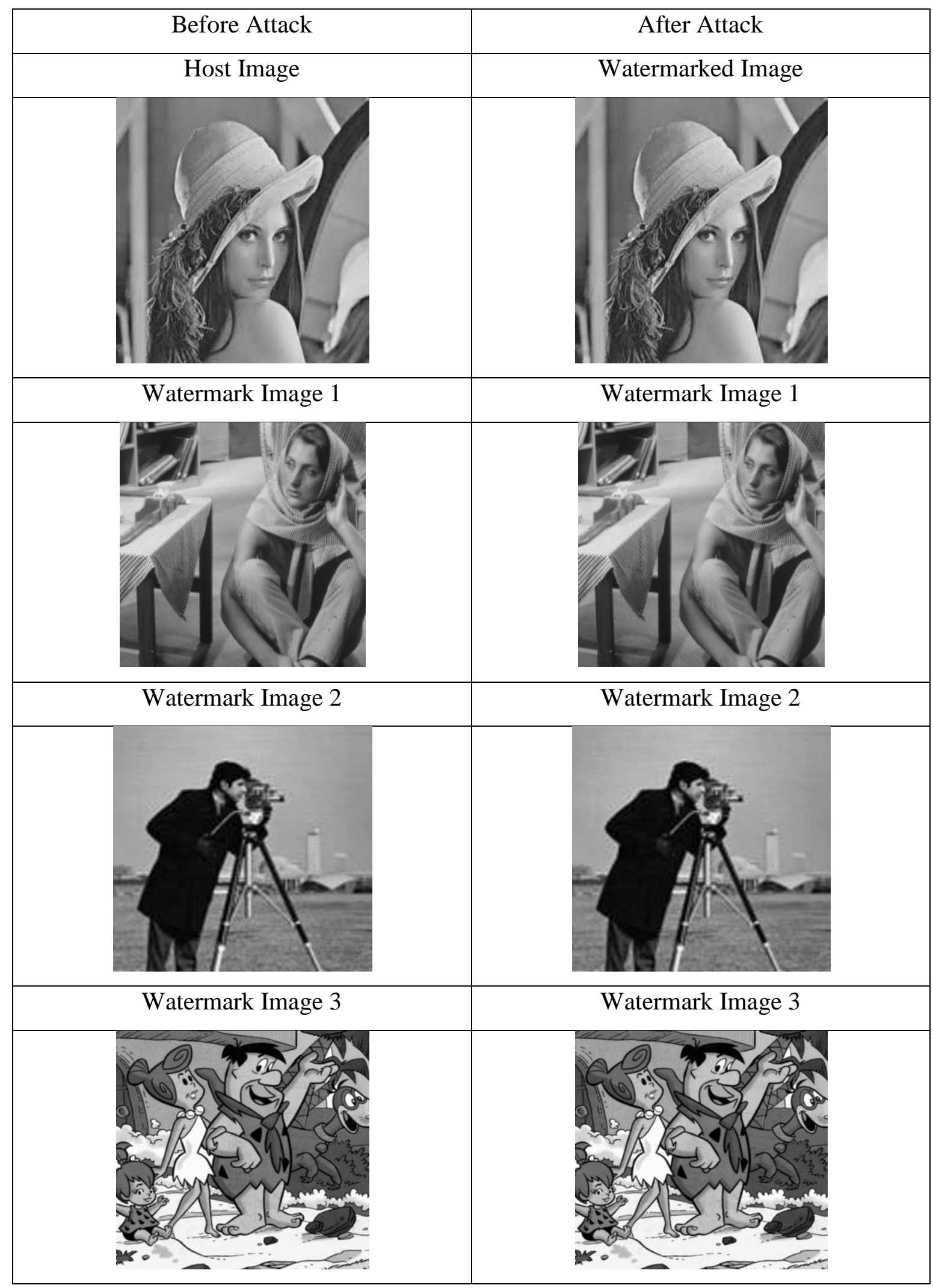

Fig.1.45: Extraction of Watermark Images against 'Mean Filtering'. 


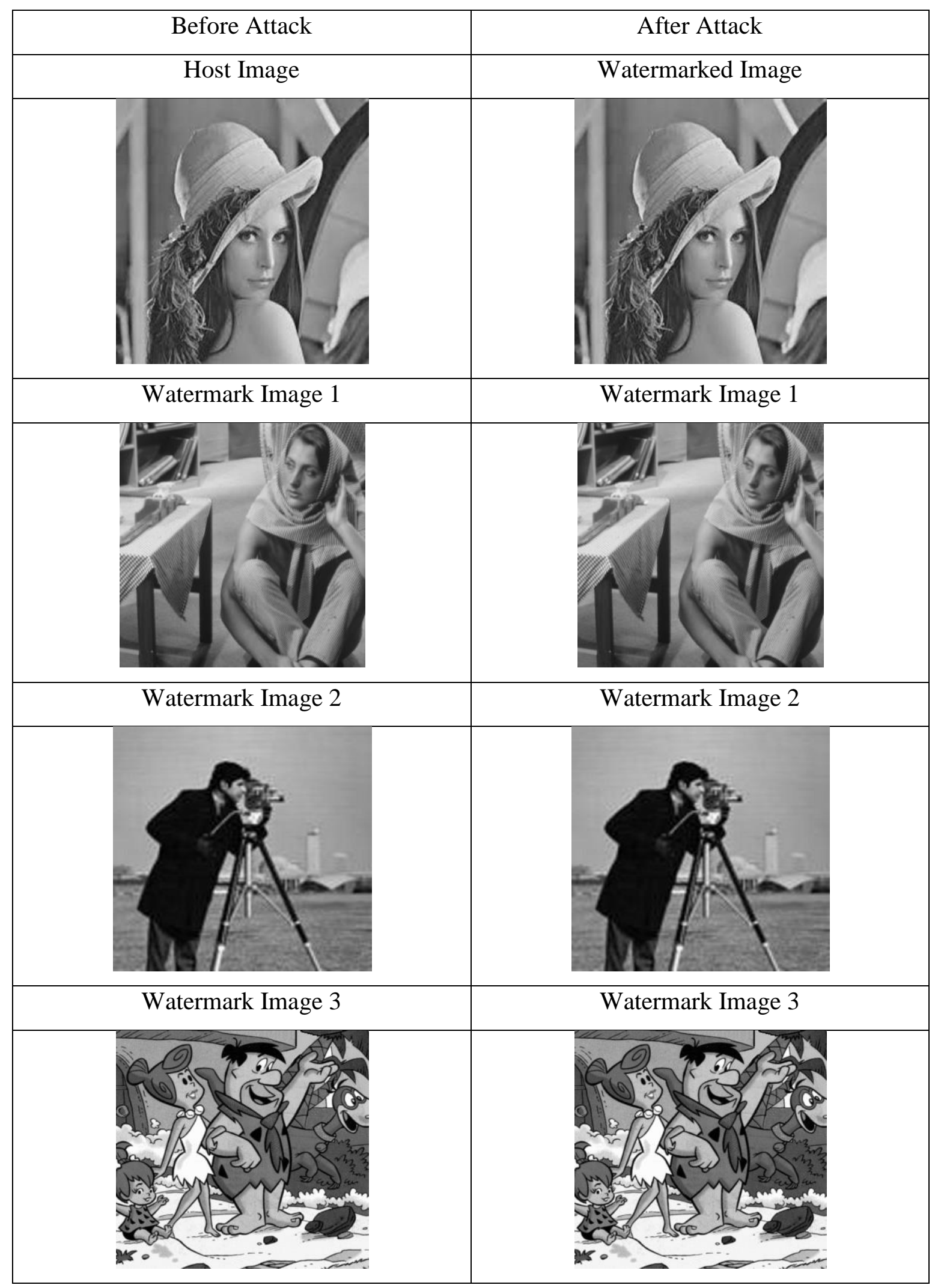

Fig.1.46: Extraction of Watermark Images against 'Gaussian Low-pass Filtering'. 


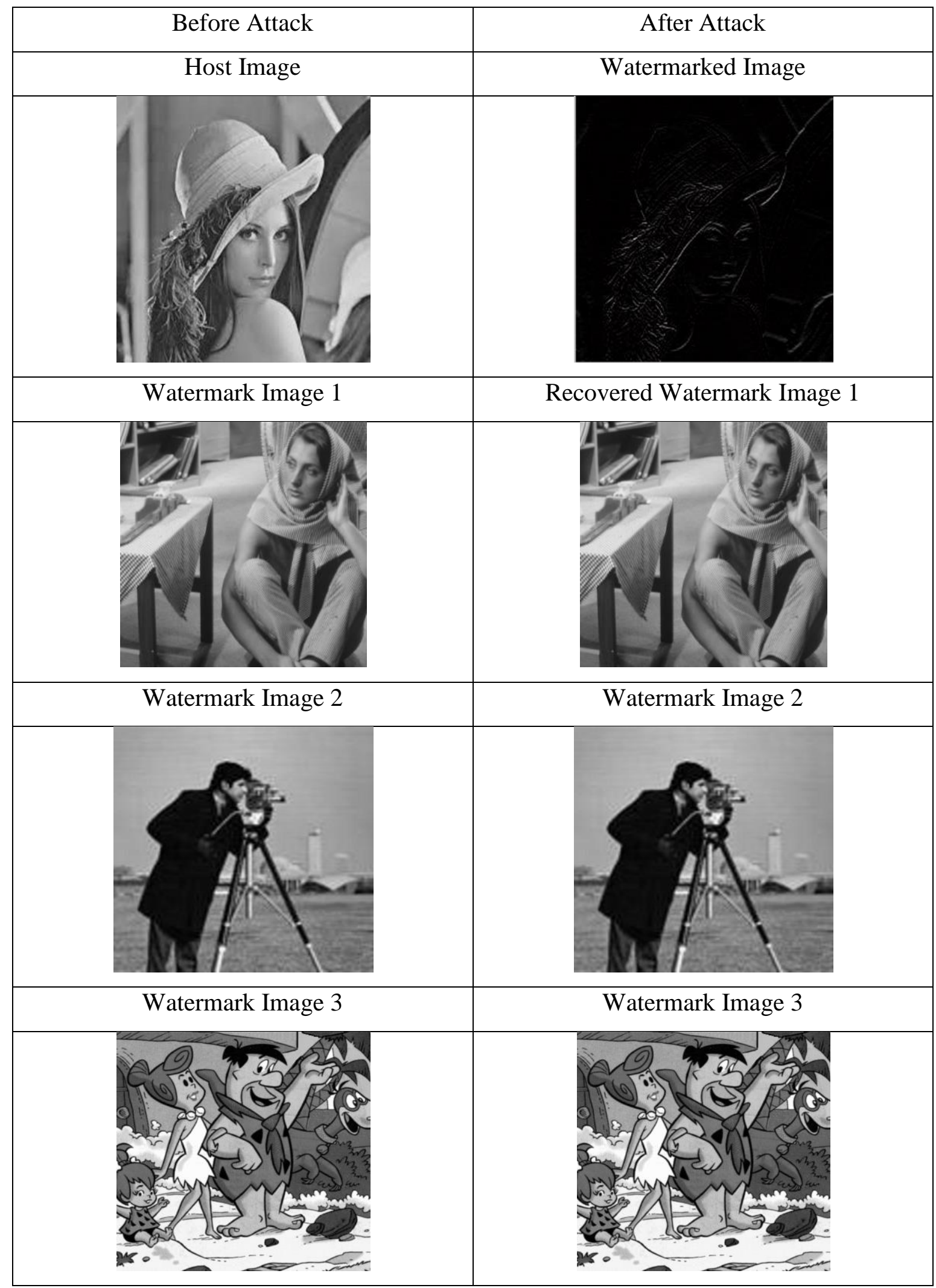

Fig.1.47: Extraction of Watermark Images against 'Prewitt Filtering'. 


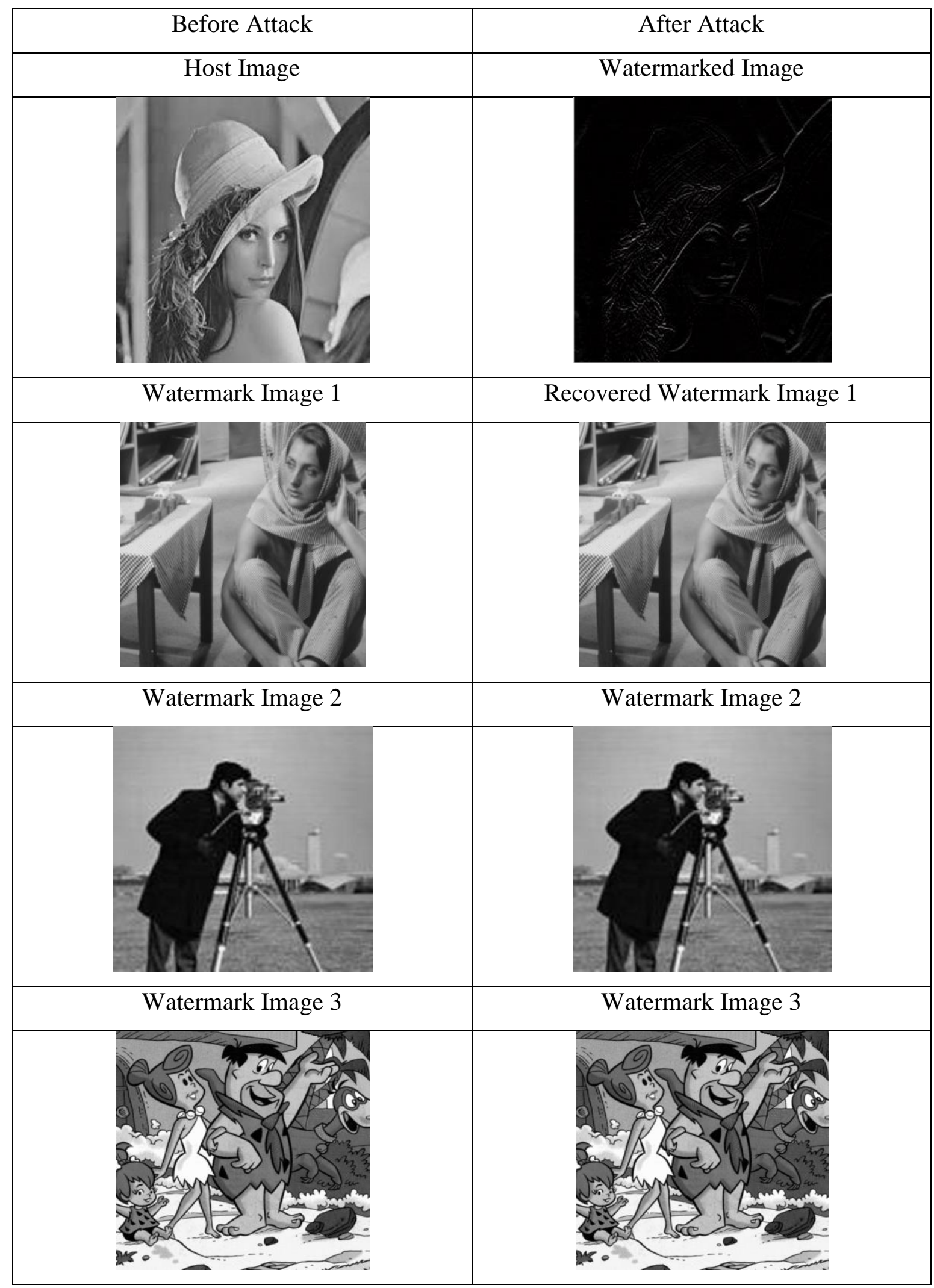

Fig.1.48: Extraction of Watermark Images against 'Sobel Filtering'. 


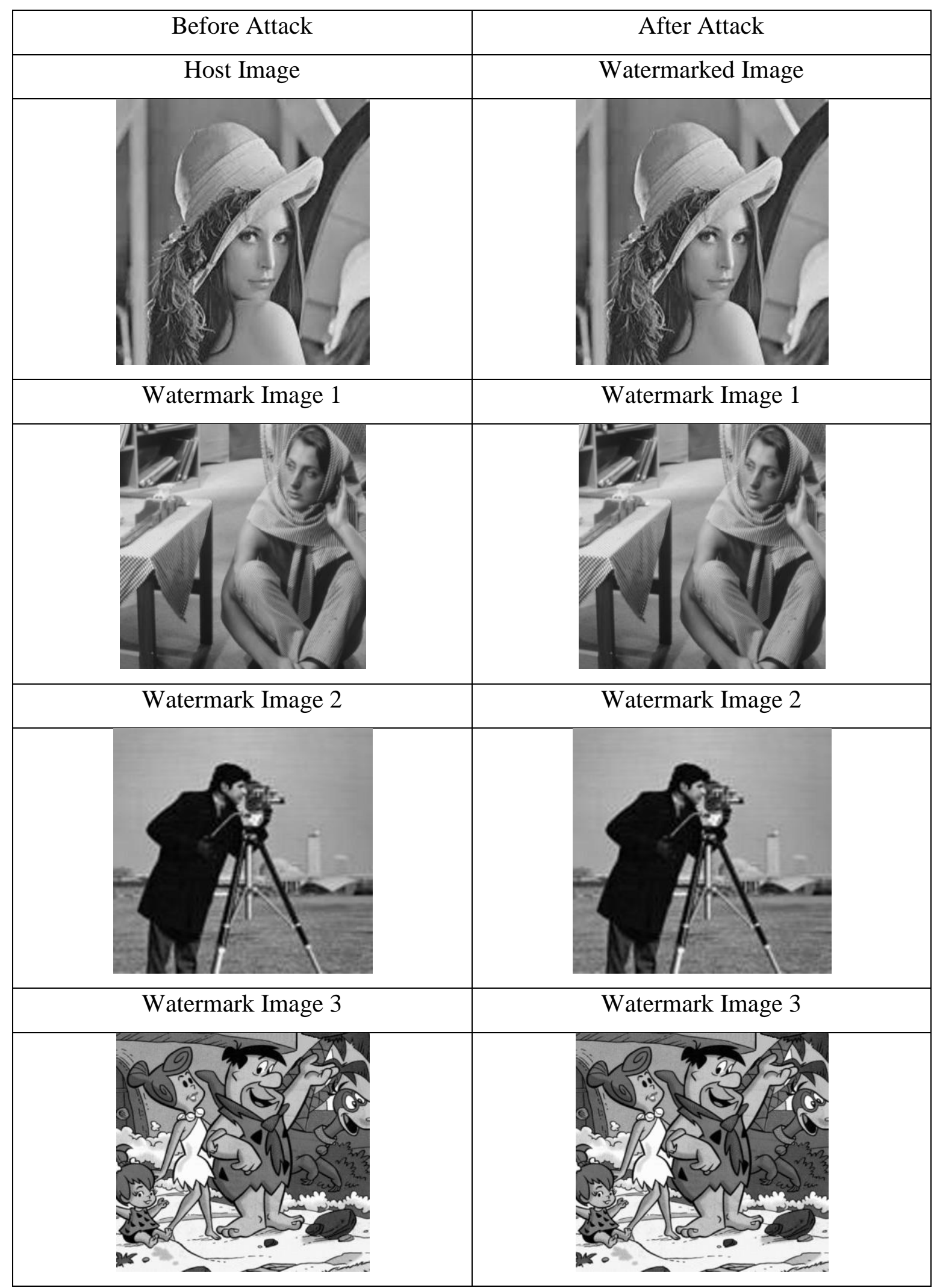

Fig.1.49: Extraction of Watermark Images against 'JPEG Compression'. 


\subsubsection{Conservation of Image Energy by Utilizing Parseval's Theorem}

In this section, the conservation of image energy before and after watermarking will be discussed by calculating the image energy by using 'Parseval's Theorem'. According to the mathematical model, the image energy should be conserved to get a higher value of PSNR and NC. This assumption will be proved by practical values.

\subsubsection{Image Energy Distribution}

\subsection{Host Image}

As has been said earlier, the size of the host image should be $\frac{1}{8}$ times the size of the mark images for the successful execution of the proposed watermarking technique. In our research work, we used standard images of size $512 \times 512$. So, some image processing was done to both the host image and to the mark images. The size of the host image was increased to 4 times to its initial value prior to decomposition.

The Energy of the host image before enhancement: $1.2132 \mathrm{e}+15$

The Energy of the host image after enhancement: $3.1055 \mathrm{e}+17$

So, by enhancement, the image energy increases to 256 times its initial value. Mathematically,

$$
E_{n}=2^{2 e} E
$$

where $E_{n}=$ Energy of the host image after enhancement.

$E=$ Energy of the host image before enhancement.

$e=$ The factor of enhancement.

Here the value of $\boldsymbol{e}$ is 4 as the image was enhanced to 4 times of its original size. In the mathematical model, it was assumed that, energy is related to the size of a signal. This statement is proved. This statement will be proved throughout this section.

Next, the image will be decomposed to its sub-bands LL1, LH1, HL1 and HH1 after 1st level of decomposition. The whole energy of the image will be distributed among its sub-bands. So,

The energy of LL1 sub-band after 1st level of decomposition: 7.7629e+16

The energy of LH1 sub-band after 1st level of decomposition: $2.8289 \mathrm{e}+12$ 
The energy of HL1 sub-band after 1st level of decomposition: $5.6005 \mathrm{e}+12$ The energy of HH1 sub-band after 1st level of decomposition: 5.2045e+10 If the summation of the energy of the sub-bands is done, we get the total energy $=7.7637 \mathrm{e}+16$. But, before decomposition the image energy was $3.1055 \mathrm{e}+17$. So, there is a loss of energy during the 1st level of decomposition and by calculation the image energy after 1st level of decomposition is $\frac{1}{4}$ times to its initial value before decomposition. Also from the value of subband energy, it is also evident that the maximum image energy is concentrated into LL1 subband as has been said earlier and the energy of HH1 sub-band is the minimum. So, insertion of watermarks into that sub-band will pose less noisy effect. Because of that, the next decompositions will be done by decomposing HH1 sub-band.

Next, HH1 sub-band achieved through the1st level of decomposition was used for further decomposition and the sub-bands LL2, LH2, HL2, and HH2 will be achieved.

The energy of LL2 sub-band after 2nd level of decomposition: 5.0207e+09

The energy of LH2 sub-band after 2nd level of decomposition: 2.9099e+09

The energy of HL2 sub-band after 2nd level of decomposition: 2.4704e+09

The energy of HH2 sub-band after 2nd level of decomposition: 2.6103e+09

The energy of sub-band HH1 after 1st level of decomposition was 5.2045e+10. After decomposition, if the energy of the sub-bands achieved by decomposing HH1 sub-bands are added, the total energy will be $1.3011 \mathrm{e}+10$ which is $\frac{1}{4}$ times of the energy of HH1 sub-band before decomposition. From the energy of the sub-bands, it is also evident here that, the LL2 sub-band contains most of the energy. But, this time the HL2 sub-band contains the minimum rather than $\mathrm{HH} 2$ sub-band. But the difference is very low. So, the next level of decomposition will be done by decomposing the HH2 sub-band and the sub-bands LL3, LH3, HL3, and HH3 will be achieved.

The energy of LL3 sub-band after 3rd level of decomposition: 1.3641e+08

The energy of LH3 sub-band after 3rd level of decomposition: 1.4669e+08

The energy of HL3 sub-band after 3rd level of decomposition: 1.6945e+08

The energy of HH3 sub-band after 3rd level of decomposition: 2.0003e+08

The total energy of $\mathrm{HH} 2$ sub-band before decomposition is $2.6103 \mathrm{e}+09$ and After decomposition, the total energy of HH2 sub-band is combining the energy of sub-bands LL3, 
LH3, HL3, and HH3 is $6.5258 \mathrm{e}+08$ which is $\frac{1}{4}$ times of the energy of HH2 sub-band before decomposition. Here also the LL3 sub-band contains the maximum image energy.

The HH3 sub-band will be further decomposed to get the sub-bands LL4, LH4, HL4, and HH4.

The energy of LL4 sub-band after 4th level of decomposition: 1.3150e+07

The energy of LH4 sub-band after 4th level of decomposition: 1.2731e+07

The energy of HL4 sub-band after 4th level of decomposition: 1.2022e+07

The energy of HH4 sub-band after 4th level of decomposition: 1.2105e+07

Before decomposition the energy of HH3 sub-band was $2.0003 \mathrm{e}+08$ and after decomposition, the energy is after combining the energy of the sub-bands is $5.0008 \mathrm{e}+07$ which is $\frac{1}{4}$ times the energy of HH3 sub-band before decomposition. Also, the energy of LL4 sub-band is the maximum. So, to locate the sub-bands of the watermark images, the LH4, HL4 and HH4 sub-bands will be further decomposed to LL5, LH5, HL5, \& HH5 sub-bands; LL6, LH6, HL6 \& HH6 sub-bands and LL7, LH7, HL7 \& HH7 sub-bands respectively. From the decomposition, it was observed that there is an amount of energy loss in every level of decomposition. So, a mathematical formula can be developed indicating the relationship between the energy before and after decomposition.

$$
E_{1}=\frac{1}{4} E_{2}
$$

where $E_{1}=$ The energy of the sub-band used for decomposition. The energy is achieved by combining the energy of the sub- bands achieved by its decomposition.

$E_{2}=$ The energy of the sub-band before used for further decomposition.

The energy of LL5 sub-band after 5th level of decomposition: 8.3792e+05

The energy of LH5 sub-band after 5th level of decomposition: 8.5482e+05

The energy of HL5 sub-band after 5th level of decomposition: 7.5146e+05

The energy of HH5 sub-band after 5th level of decomposition: 7.3853e+05

The energy of LL6 sub-band after 6th level of decomposition: 7.5178e+05

The energy of LH6 sub-band after 6th level of decomposition: 6.9386e+05

The energy of HL6 sub-band after 6th level of decomposition: 7.8164e+05

The energy of HH6 sub-band after 6th level of decomposition: 7.7824e+05 
The energy of LL7 sub-band after 7th level of decomposition: 7.3599e+05

The energy of LH7 sub-band after 7th level of decomposition: 7.3077e+05

The energy of HL7 sub-band after 7th level of decomposition: 7.7221e+05

The energy of HH7 sub-band after 7th level of decomposition: 787220

Before decomposition the energy of LH4, HL4 and HH4 sub-bands are 1.2731e+07, 1.2022e+07 and $1.2105 e+07$ respectively. After decomposition combining the respective sub-bands, their energy becomes $3.1827 \mathrm{e}+06,3.0055 \mathrm{e}+06$ and $3.0262 \mathrm{e}+06$ respectively which are $\frac{1}{4}$ times the energy before decomposition respectively. The energy of the bands used to locate the sub-bands of the watermark images are by calculation:

$$
\begin{aligned}
& E_{L H 4}=4 \times 10^{-11} E_{n} \\
& E_{H L 4}=4 \times 10^{-11} E_{n} \\
& E_{H H 4}=4 \times 10^{-11} E_{n}
\end{aligned}
$$

From these equations, it can be seen that the energy of the bands used for the location of watermark images are very low in comparison with the host image. A very small percentage of the energy of the host image was used for embedding watermarks. From the calculation throughout the decomposition process, it was also evident that every level of decomposition caused a decrease in energy. Also, the amount of decreased energy depends on the sub-band which is chosen for further decomposition. The LL sub-band contains most of the energy and the HH sub-band contains the minimum energy most of the times of decomposition. So, by choosing $\mathrm{HH}$ sub-band for further decomposition, the final energy will be very low in comparison with the host image which is evident from the energy calculation. By locating the watermarks in the lower energy level will cause lesser distortion to the host image. So, by utilizing multi-level DWT with the proper choice of sub-band for locating the sub-bands of watermark images can yield a very good result which is evident from the results shown in the previous chapters.

\subsection{Watermark Images}




\section{$1^{\text {st }}$ Watermark Image}

The size of watermark images was decreased to 2 times to its initial value prior to decomposition.

The Energy of the $1^{\text {st }}$ watermark image before size decrease: $2.3468 \mathrm{e}+15$

TheEnergy of the $1^{\text {st }}$ watermark image after size decrease: $1.4644 \mathrm{e}+14$

So, by decreasing the size of the image, the image energy decreases to $\frac{1}{16}$ times of its initial value. Mathematically,

$$
E_{n}^{W 1}=2^{2 f} E^{W 1}
$$

where, $E_{n}^{W 1}=$ Energy of the watermark image after decreasing its size.

$E^{W 1}=$ Energy of the watermark image before decreasing its size.

$f=$ The factor of decreasing the image size.

Here the value of $\boldsymbol{f}$ is 2 as the size of the image was decreased 2 times.

Next, the image will be decomposed to its sub-bands LLw1, LHw1, HLw1 and HHw1 after 1st level of decomposition. The whole energy of the image will be distributed among its sub-bands. So,

The energy of LLw1 sub-band after 1st level of decomposition: 3.6474e+13

The energy of LHw1 sub-band after 1st level of decomposition: 6.8818e+10

The energy of HLw1 sub-band after 1st level of decomposition: 5.9616e+10

The energy of HHw1 sub-band after 1st level of decomposition: 8.4429e+09

If the summation of the energy of the sub-bands is done, we get the total energy $=3.6611 \mathrm{e}+13$. But, before decomposition the image energy was $1.4644 \mathrm{e}+14$. So, the image energy after $1 \mathrm{st}$

level of decomposition is $\frac{1}{4}$ times its initial value before decomposition. So, the relationship developed by equation (1.4) is also true for the decomposition of the watermark image. Also from the values of sub-band energy, it is also evident that the maximum image energy is concentrated into LLw1 sub-band as has been said earlier and the energy of HHw1 sub-band is the minimum. So, the HHw1 sub-band will be used for a further level of decomposition.

HHw1 sub-band achieved through the1st level of decomposition was used for further decomposition and the sub-bands LLw11, LHw11, HLw11, and HHw11 will be achieved. 
The energy of LLw11 sub-band after 2nd level of decomposition: 2.6072e+08 The energy of LHw11 sub-band after 2nd level of decomposition: 4.3457e+08 The energy of HLw11 sub-band after 2nd level of decomposition: 4.6530e+08 The energy of HHw11 sub-band after 2nd level of decomposition: 9.5014e+08 These sub-bands will be used for watermarking. The relationship between the energy of the subbands used for watermarking with the energy of the watermark before decomposition can be mathematically formulated by the following equations:

$$
\begin{aligned}
E_{L L w 11} & =\left(2.0 \times 10^{-6}\right) E_{n}^{W 1} \\
E_{L H w 11} & =\left(3.0 \times 10^{-6}\right) E_{n}^{W 1} \ldots \\
E_{H L w 11} & =\left(3.2 \times 10^{-6}\right) E_{n}^{W 1} \\
E_{H H w 11} & =\left(6.5 \times 10^{-6}\right) E_{n}^{W 1}
\end{aligned}
$$

From the above energy distribution, it is seen that very low energy components of mark image are embedded in the host image.

The relationship between the energy of the sub-bands of the mark images used for watermarking with the energy of the host image before decomposition can be mathematically formulated by the following equations:

$$
\begin{aligned}
& E_{L L w 11}=\left(8.4 \times 10^{-10}\right) E_{n} \\
& E_{L H w 11}=\left(1.4 \times 10^{-9}\right) E_{n} \\
& E_{H L w 11}=\left(1.5 \times 10^{-9}\right) E_{n} \\
& E_{H H w 11}=\left(3.1 \times 10^{-9}\right) E_{n}
\end{aligned}
$$

From the equations, it is evident that the energy of the sub-bands of the mark image is very much low in comparison with the energy of the host image. This little energy can hardly make any distortion to the host image.

\section{2nd Watermark Image}

The energy of the 2nd watermark image before size decrease: $4.4938 \mathrm{e}+14$

The energy of the 2nd watermark image after size decrease: $2.7957 \mathrm{e}+13$

So, by decreasing the size of the image, the image energy decreases to $\frac{1}{16}$ times of its initial value. The equation (1.8) holds true for the second watermark image. 
Next, the image will be decomposed to its sub-bands LLw2, LHw2, HLw2 and HHw2 after 1st level of decomposition. The whole energy of the image will be distributed among its sub-bands. So,

The energy of LLw2 sub-band after 1st level of decomposition: 6.9549e+12

The energy of LHw2 sub-band after 1st level of decomposition: 1.5171e+10

The energy of HLw2 sub-band after 1st level of decomposition: 1.6300e+10

The energy of HHw2 sub-band after 1st level of decomposition: 2.7760e+09

If the summation of the energy of the sub-bands is done, we get the total energy $=6.9892 \mathrm{e}+12$. But, before decomposition the image energy was $2.7957 \mathrm{e}+13$. So, the image energy after $1 \mathrm{st}$

level of decomposition is $\frac{1}{4}$ times to its initial value before decomposition. So, the relationship developed by equation (1.4) is also true for the decomposition of the watermark image. Also from the values of sub-band energy, it is also evident that the maximum image energy is concentrated into LLw2 sub-band and the HHw2 sub-band contains the minimum image energy. So, HHw2 sub-band achieved through the 1st level of decomposition was used for further decomposition and the sub-bands LLw22, LHw22, HLw22, and HHw22 will be achieved.

The energy of LLw22 sub-band after 2nd level of decomposition: 8.5398e+07

The energy of LHw22 sub-band after 2nd level of decomposition: 1.7143e+08

The energy of HLw22 sub-band after 2nd level of decomposition: 1.5430e+08

The energy of HHw22 sub-band after 2nd level of decomposition: 282865152

These sub-bands will be used for watermarking. The relationship between the energy of the subbands used for watermarking with the energy of the watermark before decomposition can be mathematically formulated by the following equations:

$$
\begin{aligned}
E_{L L w 22} & =\left(3.1 \times 10^{-6}\right) E_{n}^{W 2} \ldots \\
E_{L H w 22} & =\left(6.1 \times 10^{-6}\right) E_{n}^{W 2} \ldots \\
E_{H L w 22} & =\left(5.5 \times 10^{-6}\right) E_{n}^{W 2} \\
E_{H H w 22} & =\left(1.1 \times 10^{-5}\right) E_{n}^{W 2}
\end{aligned}
$$

where $E_{n}^{W 2}$ is the energy of the second watermark image before any level of decomposition was done.

From the above energy distribution, it is seen that very low energy components of mark image are embedded in the host image. 
The relationship between the energy of the sub-bands of the mark image used for watermarking with the energy of the host image before decomposition can be mathematically formulated by the following equations:

$$
\begin{aligned}
E_{L L w 22} & =\left(2.7 \times 10^{-10}\right) E_{n} \\
E_{L H w 22} & =\left(5.5 \times 10^{-10}\right) E_{n} \\
E_{H L w 22} & =\left(5.0 \times 10^{-10}\right) E_{n} \\
E_{H H w 22} & =\left(9.1 \times 10^{-10}\right) E_{n}
\end{aligned}
$$

In the case of the second watermark, also the energy of its bands is very low in comparison with that of the host image causing lesser distortion.

\section{$3^{\text {rd }}$ Watermark Image}

The Energy of the 3rd watermark image before size decrease: $1.6643 \mathrm{e}+15$

The energy of the 3rd watermark image after size decrease: $1.0293 \mathrm{e}+14$

So, by decreasing the size of the image, the image energy decreases to $\frac{1}{16}$ times of its initial value. So, the relationship developed by equation (1.8) can be applicable to the third watermark image too.

Next, the image will be decomposed to its sub-bands LLw3, LHw3, HLw3 and HHw3 after 1st level of decomposition. The whole energy of the image will be distributed among its sub-bands. So,

The energy of LLw3 sub-band after 1st level of decomposition: $2.5154 \mathrm{e}+13$

The energy of LHw3 sub-band after 1st level of decomposition: 2.7356e+11

The energy of HLw3 sub-band after 1st level of decomposition: 2.5141e+11

The energy of HHw3 sub-band after 1st level of decomposition: $5.3055 \mathrm{e}+10$

If the summation of the energy of the sub-bands is done, we get the total energy $=2.5732 \mathrm{e}+13$. But, before decomposition the image energy was $1.0293 \mathrm{e}+14$. So, the image energy after $1 \mathrm{st}$ level of decomposition is $\frac{1}{4}$ times to its initial value before decomposition which can be mathematically formulated by equation (1.4).Also from the values of sub-band energy, it is also evident that the maximum image energy is concentrated into LLw3 sub-band and the HHw3 subband contains the minimum image energy. So, HHw3 sub-band achieved through the 1st level of 
decomposition was used for further decomposition and the sub-bands LLw33, LHw33, HLw33, and HHw33 will be achieved.

The energy of LLw33 sub-band after 2nd level of decomposition: 1.1849e+09

The energy of LHw33 sub-band after 2nd level of decomposition: 2.6932e+09

The energy of HLw33 sub-band after 2nd level of decomposition: 2.7164e+09

The energy of HHw33 sub-band after 2nd level of decomposition: 6.6693e+10

These sub-bands will be used for watermarking. The relationship between the energy of the subbands used for watermarking with the energy of the watermark before decomposition can be mathematically formulated by the following equations:

$$
\begin{aligned}
E_{L L w 33} & =\left(1.2 \times 10^{-5}\right) E_{n}^{W 3} \\
E_{L H w 33} & =\left(2.6 \times 10^{-5}\right) E_{n}^{W 3} \\
E_{H L w 33} & =\left(2.6 \times 10^{-5}\right) E_{n}^{W 3} \\
E_{H H w 33} & =\left(6.5 \times 10^{-4}\right) E_{n}^{W 3}
\end{aligned}
$$

where $E_{n}^{W 3}$ is the energy of the third watermark image before any level of decomposition was done.

From the above energy distribution, it is seen that very low energy components of mark image are embedded in the host image.

The relationship between the energy of the sub-bands used for watermarking with the energy of the watermark before decomposition can be mathematically formulated by the following equations:

$$
\begin{aligned}
E_{L L w 33} & =\left(3.8 \times 10^{-9}\right) E_{n} \ldots \ldots \ldots \ldots \ldots \ldots \ldots \ldots(1.29) \\
E_{L H w 33} & =\left(8.7 \times 10^{-9}\right) E_{n} \ldots \ldots \ldots \ldots \ldots \ldots \ldots \ldots(1.30) \\
E_{H L w 33} & =\left(8.7 \times 10^{-9}\right) E_{n} \ldots \ldots \ldots \ldots \ldots \ldots \ldots \ldots(1.31) \\
E_{H H w 33} & =\left(2.1 \times 10^{-7}\right) E_{n} \ldots \ldots \ldots \ldots \ldots \ldots \ldots(1.32)
\end{aligned}
$$

Here also very low energy sub-bands of the mark image were used for the watermarking process. From the energy distribution of the mark images, it can be observed that, after 1st level of decomposition, the energy of the LL sub-band was maximum among the sub-bands achieved through the 1st level of decomposition and this is true for all the mark images. This is desired as it is known that maximum image energy is concentrated on low-frequency sub-band. But, when the HH sub-band was decomposed to get another four sub-band i.e. 2nd level of decomposition 
was done, the energy of the HH sub-band achieved by the 2nd level of decomposition became maximum among the other sub-bands. So, a shift of energy happened during the decomposition process. So, mathematically after decomposition,

$$
\begin{aligned}
& E_{H H w 11}=3.6 \times E_{L L w 11} \ldots \ldots \ldots \ldots \ldots \ldots \ldots \ldots(1.33) \\
& E_{H H w 22}=3.3 \times E_{L L w 22} \ldots \ldots \ldots \ldots \ldots \ldots \ldots \ldots(1.34) \\
& E_{H H w 33}=56 \times E_{L L w 33} \ldots \ldots \ldots \ldots \ldots \ldots \ldots \ldots(1.35)
\end{aligned}
$$

Now, combining the energy of sub-bands LLw11, LHw11, HLw11, and HHw11, we get, $2.1 \times$ $10^{9}$. Again, combining the energy of sub-bands LLw22, LHw22, HLw22 and HHw22, we get, $6.9 \times 10^{9}$. Further, combining the energy of sub-bands LLw33, LHw33, HLw33, and HHw33, we get, $7.3 \times 10^{10}$. So, the total energy of the mark images which will be inserted into the host image will be the addition of the energy of all these sub-bands. So, the inserted energy is $8.2 \times$ $10^{10}$. The energy of the host image is, $3.1055 \times 10^{17}$. So, the SNR (Signal to Noise Ratio) will be,

$$
\begin{gathered}
S N R=20 \log \frac{3.1055 \times 10^{17}}{8.2 \times 10^{10}} \\
=131.6 \mathrm{~dB}
\end{gathered}
$$

which is very close to the value achieved by practical implementation.

\subsection{Watermarked Image}

After DWT, SVD was done to selected sub-bands of the host image and watermark images for getting the marked image. The combination of the host image and mark images were done by a procedure which was discussed in detail in section 1.2.1, 1.2.2 and 1.2.3 of the chapter 'Image Watermarking'. After combination, the energy of the sub-bands used for locating the sub-bands of the mark images is:

The energy of LL5 sub-band after embedding the watermark: 1.7971e+04

The energy of LH5 sub-band after embedding the watermark: 5.9652e+04

The energy of HL5 sub-band after embedding the watermark: $6.3250 \mathrm{e}+03$

The energy of HH5 sub-band after embedding the watermark: 1.5402e+04

The energy of LL6 sub-band after embedding the watermark: 295.6641

The energy of LH6 sub-band after embedding the watermark: 3.6482e+03

The energy of HL6 sub-band after embedding the watermark: 0 
The energy of HH6 sub-band after embedding the watermark: 837.7250

The energy of LL7 sub-band after embedding the watermark: $2.4254 \mathrm{e}+05$

The energy of LH7 sub-band after embedding the watermark: $6.4573 \mathrm{e}+05$

The energy of HL7 sub-band after embedding the watermark: 8.8582e+04

The energy of HH7 sub-band after embedding the watermark: 2.3892e+05

Also, the energy of the sub-bands not used for embedding watermarks are:

The energy of LL4 sub-band after embedding the watermark: 0

The energy of LL3 sub-band after embedding the watermark: 0

The energy of LH3 sub-band after embedding the watermark: 0

The energy of HL3 sub-band after embedding the watermark: 0

The energy of HH3 sub-band after embedding the watermark: 0

The energy of LL2 sub-band after embedding the watermark: 0

The energy of LH2 sub-band after embedding the watermark: 0

The energy of HL2 sub-band after embedding the watermark: 0

The energy of $\mathrm{HH} 2$ sub-band after embedding the watermark: 0

The energy of LL1 sub-band after embedding the watermark: 7.7625e+16

The energy of LH1 sub-band after embedding the watermark: $1.6275 \mathrm{e}+12$

The energy of HL1 sub-band after embedding the watermark: 4.3440e+12

The energy of HH1 sub-band after embedding the watermark: 0

Before decomposition the energy of LH4, HL4 and HH4 sub-bands are 1.2731e+07, 1.2022e+07 and $1.2105 \mathrm{e}+07$ respectively. After decomposition combining the respective sub-bands, their energy becomes 3.1827e+06, 3.0055e+06 and 3.0262e+06 respectively. After embedding watermarks the energy of the sub-bands by combining the energy of the respective sub-bands became $9.9350 \mathrm{e}+04,4.7816 \mathrm{e}+03$, and $1.2158 \mathrm{e}+06$. So, it is seenthat after embedding the energy of the sub-bands of the host image used for inserting watermark images has decreased as the 
mark images posed noisy effects on the host image. Also, it is seen that the energy of the subband LL4, HH1 and the sub-bands achieved through the second and third level of decomposition is zero. But, the energy of LL1, LH1, and HL1 sub-bands are very near to the energy before embedding watermark. If we combine all the sub-band energy we get total energy of $7.76 \times$ $10^{16}$ which is $\frac{1}{4}$ times the host energy before decomposition. Mathematically,

$$
E_{W M}=\frac{1}{4} \times E_{n} \ldots \ldots \ldots \ldots \ldots \ldots \ldots \ldots \ldots \ldots \ldots \ldots \ldots
$$

where $E_{W M}=$ Energy of the marked image achieved by combining the energy of all the sub-bands achieved by $1^{\text {st }}$ to $7^{\text {th }}$ level of decomposition.

It is also evident that most of the energy is concentrated to LL1 sub-band even after embedding the mark images and the insertion of mark images have a considerable amount of effect on the energy distribution through the sub-bands not even used for the embedding of the watermarks. Also, the energy of the marked image is $\frac{1}{4}$ times the energy of the host image by the equation (1.36) i.e. $\frac{3}{4}$ of the energy of the host, image is lost during the decomposition process.

But, after embedding procedure is done, the energy of the marked image is calculated $3.1053 \mathrm{e}+17$ which is almost same as the energy of the host image $3.1055 \mathrm{e}+17$ before doing any kind of processing like DWT, DCT or SVD. How can this be possible when there exists an energy loss? The answer lies in the calculation of the energy of every level of decomposition using DWT after embedding the mark images.

The energy of $7^{\text {th }}$ Level of Decomposition: 4.8631e+06

The energy of $6^{\text {th }}$ Level of Decomposition: $1.9126 \mathrm{e}+04$

The energy of $5^{\text {th }}$ Level of Decomposition: $3.9740 \mathrm{e}+05$

Energy of $4^{\text {th }}$ Level of Decomposition: $2.1119 \mathrm{e}+07$

The energy of $3^{\text {rd }}$ Level of Decomposition: $8.4474 \mathrm{e}+07$

The energy of $2^{\text {nd }}$ Level of Decomposition: $3.3790 \mathrm{e}+08$

The energy of $1^{\text {st }}$ Level of Decomposition: $3.1053 \mathrm{e}+17$

By combination, the energy becomes $3.1053 \mathrm{e}+17$ which is close to the actual energy of the host image. So, there is always an existence of energy loss due to the insertion of mark images into the host image. There is also a loss of energy due to decomposition which was discussed before. But, if the embedding algorithm is strong enough, the loss of energy introduced by the mark images can be reduced to a negligible amount which is evident from the value of PSNR of the 
proposed technique. And the value of the coefficient taken as 0.01 was also right as by this coefficient a very small fraction of watermark is inserted reducing the energy loss of the mark image. So, to embed one watermark $\frac{1}{4}$ of the energy of the host, image will be necessary. As three watermarks were used, $\frac{3}{4}$ of the energy of the host, image was necessary. Mathematically,

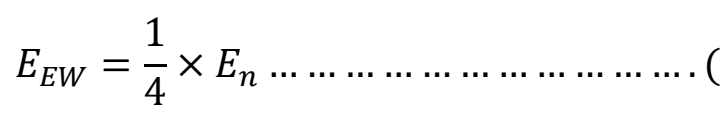

where $E_{E W}=$ The energy required for embedding one watermark.

But, if we consider equation (1.4), it can be seen that the amount of energy loss was introduced by every level of decomposition and that was also $\frac{3}{4}$ of the energy of the host image. By a level of decomposition, four sub-bands can be achieved. So, to get one sub-band $\frac{3}{4}$ of the energy of the host, image is necessary that is why the equation (1.4) is derived. But, by observing equation (1.37), the effect of equation (1.4) is observed. So, some decisions can be made. First of all, there is no energy needed for embedding the watermarks otherwise the factor of energy loss will be higher than $\frac{3}{4}$ which is not observed by the equation (1.38). This is because the value of coefficient was 0.001 for embedding the watermarks by equation (1.1). By using this value, a very small portion of watermark was embedded in the host image. As the portion is low, the energy is low also. Combining this low energy with the host image caused almost no energy loss which is evident from the value of PSNR. The use of 0.001 as combination co-efficient was right as by using this value, the mark images were hardly able to cause a noisy effect. The insertion of the mark images can have an effect on the energy distribution of the sub-bands not used for embedding. But, cannot change the total energy distribution of the host image if the embedding algorithm is strong enough which is evident from the energy of the watermarked image calculated by using 'Parseval's Theorem'.

\subsection{Extraction of Watermark Images}

\section{1st Watermark}

The energy of LLw1 sub-band after extraction: 3.6472e+13

The energy of LHw1 sub-band after extraction: $6.4452 \mathrm{e}+10$

The energy of HLw1 sub-band after extraction: 5.5171e+10

The energy of HHw1 sub-band after extraction: 4.1556e+09 
HHw1 sub-band achieved through the 1st level of decomposition was used for further decomposition and the sub-bands LLw11, LHw11, HLw11, and HHw11 will be achieved.

The energy of LLw11 sub-band after extraction: 2.9004e+08

The energy of LHw11 sub-band after extraction: $2.7713 \mathrm{e}+08$

The energy of HLw11 sub-band after extraction: 5.3762e+08

The energy of HHw11 sub-band after extraction: 3.2726e+08

The energy of the $1^{\text {st }}$ watermark image before embedding: $1.4644 \mathrm{e}+14$

The energy of the $1^{\text {st }}$ watermark image after extraction: $1.4639 \mathrm{e}+14$

\section{$2^{\text {nd }}$ Watermark}

The energy of LLw2 sub-band after extraction: 6.9505e+12

The energy of LHw2 sub-band after extraction: 1.0480e+10

The energy of HLw2 sub-band after extraction: 1.1722e+10

The energy of HHw2 sub-band after extraction: $2.4524 \mathrm{e}+08$

HHw2 sub-band achieved through the 1st level of decomposition was used for further decomposition and the sub-bands LLw22, LHw22, HLw22, and HHw22 will be achieved.

The energy of LLw22 sub-band after extraction: 3.5201e+07

The energy of LHw22 sub-band after extraction: 5.0487e+07

The energy of HLw22 sub-band after extraction: 2.8029e+07

The energy of HHw22 sub-band after extraction: 8.0420e+07

The energy of the 2nd watermark image before embedding: $2.7957 \mathrm{e}+13$

The energy of the 2nd watermark image after extraction: $2.7894 \mathrm{e}+13$

\section{$3^{\text {rd }}$ Watermark:}

The energy of LLw3 sub-band after extraction: $2.5153 \mathrm{e}+13$

The energy of LHw3 sub-band after extraction: 2.7080e+11

The energy of HLw3 sub-band after extraction: $2.4859 \mathrm{e}+11$

The energy of HHw3 sub-band after extraction: 4.8549e+10

HHw3 sub-band achieved through the 1st level of decomposition was used for further decomposition and the sub-bands LLw33, LHw33, HLw33, and HHw33 will be achieved. 
The energy of LLw33 sub-band after extraction: 5.2017e+07

The energy of LHw33 sub-band after extraction: $1.0526 \mathrm{e}+08$

The energy of HLw33 sub-band after extraction: 9.7223e+07

The energy of HHw33 sub-band after extraction: $1.0514 \mathrm{e}+08$

The energy of the 3rd watermark image before embedding: $1.0293 \mathrm{e}+14$

The energy of the 3rd watermark image after extraction: $1.0270 \mathrm{e}+14$

From the values of the sub-band energy, it is seen that there is slight change i.e. the energy changes due to the extraction of the mark images. The change is comparatively high in the subbands used for watermarking processes. If there is an increase in sub-band energy, then there is also a decrease in another sub-band energy. But, due to the strong extraction algorithm the total energy does not change i.e. the loss of energy in one level is overcome by the increase of energy in the next level as multi-level DWT was used. That is why very high correlation value was achieved. So, the value of the coefficient taken during the extraction procedure was right.

\subsubsection{Conclusion}

In the proposed methodology, the watermark images are embedded into the host image by decomposing the host image and the watermark images into sub-bands using DWT. Then, the DCT coefficients and the singular values of sub-bands of host image and watermark images are modified. Finally, the host image and the watermark images are combined by keeping in mind the image energy density in each sub-band so that the watermarked image can keep its invisibility. The proposed methodology worked very well under many attacks and filtering. The proposed technique is also tested for one and two watermarks to compare the results with the results obtained by using three watermarks. There is no change in the value of PSNR achieved by using one and two watermarks than the value of PSNR achieved by using three watermarks. Above all, the values of PSNR and NC are very close to the values which were assumed in developing the mathematical modeling and also the one found by calculating the energy of the host signal as well as the energy of the mark signals. The implementation of the proposed technique can take some time as the proposed methodology used three watermarks and three transform domain functions. This can be a limitation of the proposed technique. But, in today's world, the capability of the computers is high enough to calculate very much long algorithm in the blink of an eye. So, that limitation can hardly be a problem. 


\subsubsection{Comparative Analysis}

\section{Comparison with the Work Described in [1]}

In the work described in [1], the authors developed a new embedding algorithm incorporating DWT and DCT. In their method, the authors implemented 4 level DWT on the host image to decompose it. Then, they applied DCT on the HL and LH sub-bands achieved by the fourth level of decomposition. They also applied DCT on the mark image. The proposed algorithm uses two terms for embedding: the row-wise mean value of the host image and the 'Standard Deviation' of the host image. These two terms are multiplied with the watermark image by a coefficient. The mathematical formula is given below:

$$
f_{I}(m n)=f(m, n)+\alpha \times w \frac{f(m . n)-f_{\text {avg }}(m)}{\sqrt{f(m, n)^{2}-f_{\text {avg }}(m)^{2}}} \ldots \ldots \ldots \ldots \ldots \text { (1.38) }
$$

where $f(m, n)$ is host image, $f_{\text {avg }}(m)$ is the row-wise mean value of the host image, $w$ is watermark image and $f_{I}(m n)$ is the embedded image. The value of $\alpha$ was taken as 1 . The authors also implemented their work for 3 level and 2 level DWT also. In our work of image watermarking where three other images were used as watermarks, we used 4 level DWT to decompose the host image by decomposing $\mathrm{HH}$ sub-band. $5^{\text {th }}$, 6th, and $7^{\text {th }}$ level, de-composition was done by decomposing LH, HL and HH sub-bands achieved by the $4^{\text {th }}$ level of decomposition. The sub-bands achieved by $5^{\text {th }}, 6^{\text {th }}$ and $7^{\text {th }}$ level of decomposition were used to locate the sub-bands of the watermarks. We applied DCT on every sub-bands of the host and mark images but applied SVD on those sub-bands which were assigned for developing watermarking techniques. After SVD, the sub-bands were combined by following a procedure which was already described. We used an additive algorithm for embedding. We also used a coefficient for embedding. But, the value of that co-efficient was changed during the extraction procedure.

In the work described in [1], the authors tested the immunity of their proposed algorithm by applying AWGN (Additive White Gaussian Noise) attack on the marked image. So, for comparative analysis, we tested our proposed technique against AWGN with varying SNR. Also 
as we used multi-level DWT, we will compare our results with the results obtained by applying 4 level DWT of the work described in [1]. Figure 1.50 and 1.51 represent graphically the comparison between the two techniques regarding the value of PSNR and correlation without applying attack. The authors of [1] did not mention the SNR of the AWGN. So, we applied the SNR value ranges from $10 \mathrm{db}$ to $-1 \mathrm{~dB}$ with an interval of $1 \mathrm{~dB}$ and compared the results with the work described in [1]. In [1], the value of PSNR after applying AWGN is $30.21 \mathrm{~dB}$ and the value of correlation 0.8 .

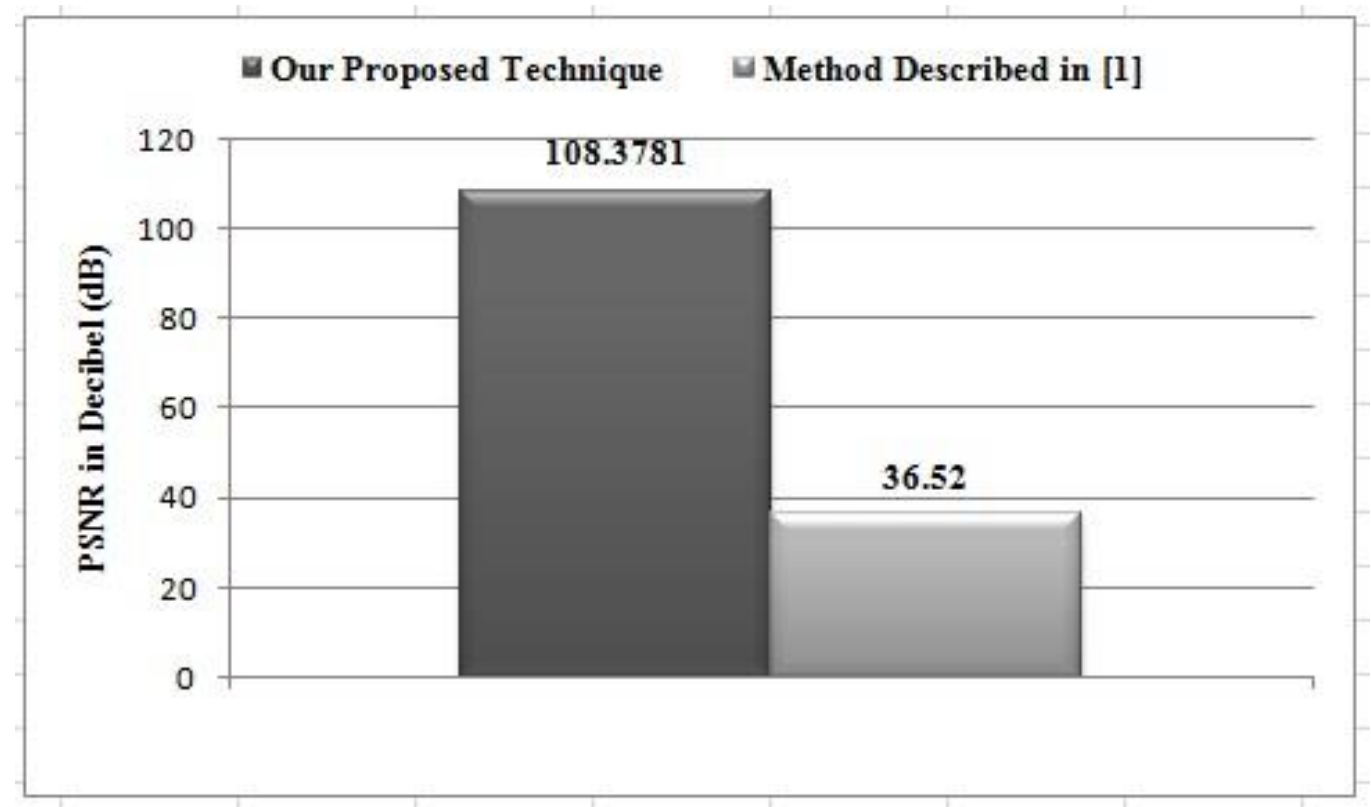

Fig. 1.50: Value of PSNR of both the Techniques. 


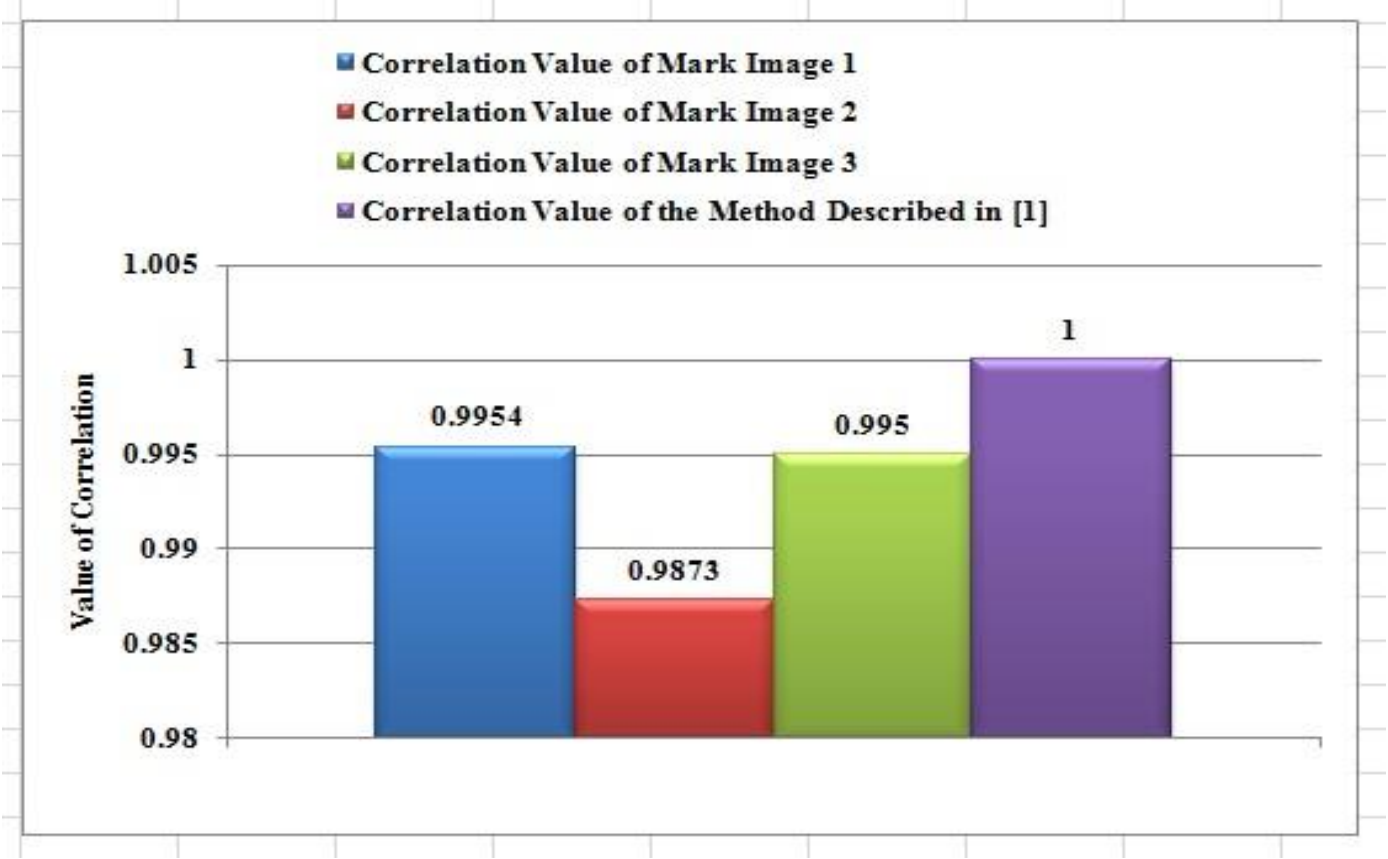

Fig.1.51: Value of Correlation of both the Techniques.

Figures 1.52 and 1.53 graphically represent the change of PSNR and correlation respectively with the change in SNR of AWGN of our proposed technique.

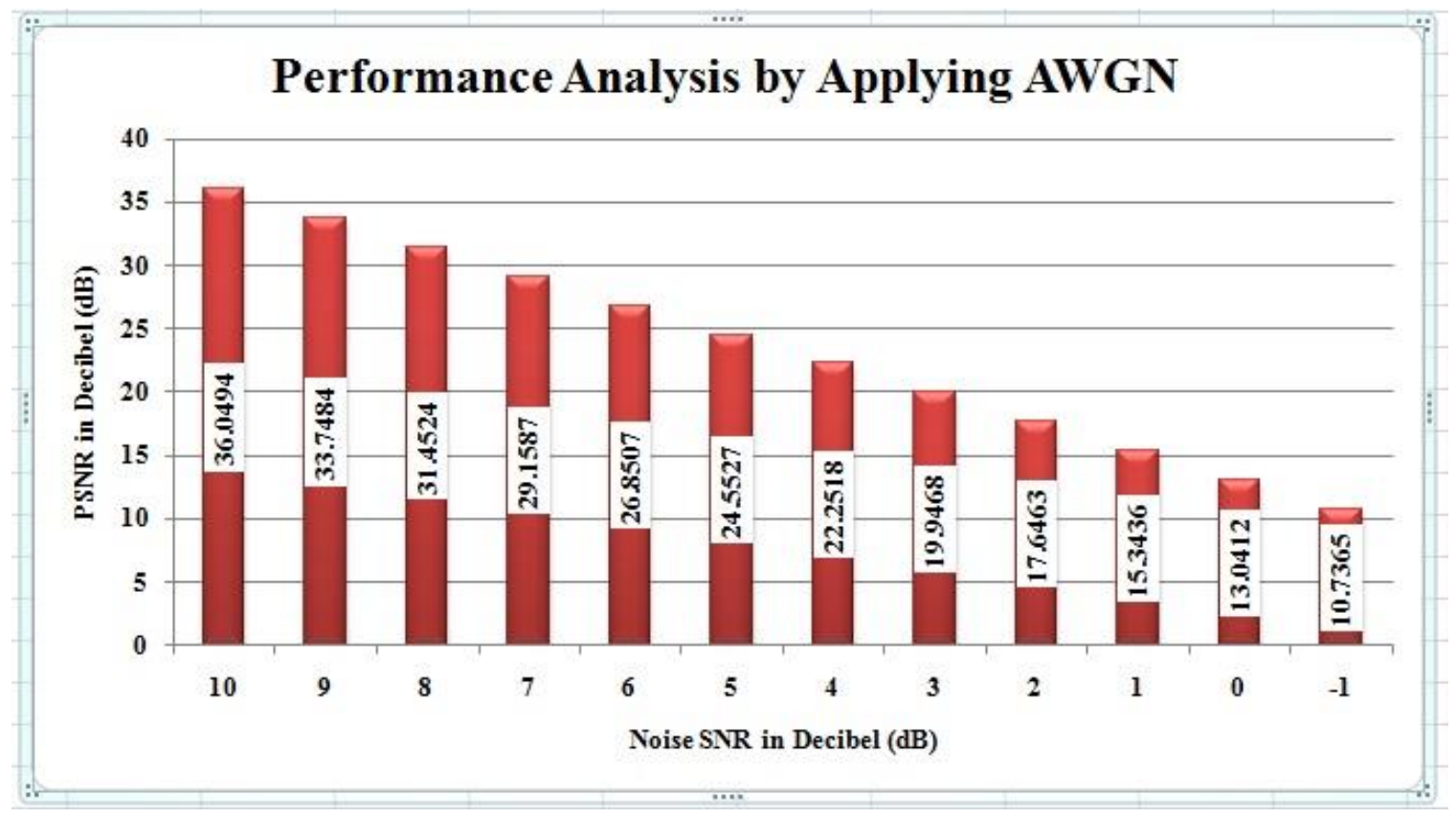

Fig. 1.52: Change in the Value of PSNR with the Change in the Value of SNR of AWGN. 


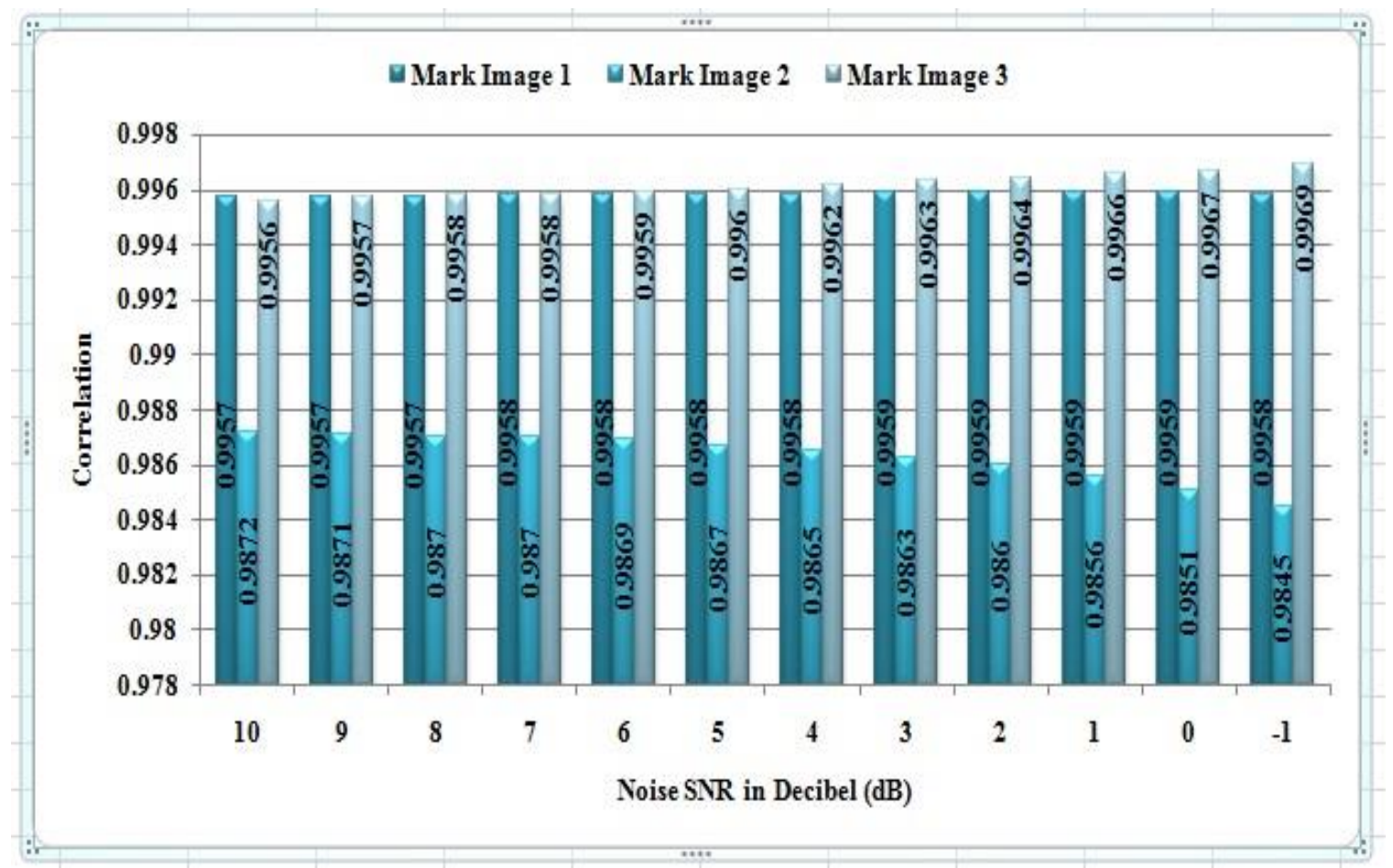

Fig. 1.53: Change in the Value of Correlation with the Change in the Value of SNR of AWGN.

From the above comparison, it is evident that our proposed technique is immune to AWGN even when the noise power is greater than the signal power. In this comparison, an impartial comparison cannot be made as the authors of [1] did not mention anythingabout the SNR of AWGN. But, in comparison with the value given, we got better correlation after applying attack. Before applying the attack, we got better PSNR value which is evident from figure 1.50. But, we got slightly less value of correlation before applying attack which is evident from figure 1.51.

\section{Comparison with the Work Described in [18]}

In this work, the authors developed an image watermarking technique using DWT, APDCBT (all phase discrete cosine bi-orthogonal transform) and SVD. The authors applied $1^{\text {st }}$ level DWT using 'Haar' wavelet. Then APDCBT was applied on the LH and HL sub-bands. After that, SVD was applied. The authors used one watermark two times. The singular values of the watermarks were inserted into the host image by using a scaling factor. A comparison was made between the work [18] and our proposed technique for image watermarking using three images as watermarks. In our method, we applied 4 level DWT using 'Daubechies' wavelet to the host image by decomposing $\mathrm{HH}$ sub-bands. $5^{\text {th }}, 6^{\text {th }}$ and $7^{\text {th }}$ level decompositions were obtained by 
decomposing LH, HL and HH sub-bands obtained by $4^{\text {th }}$ level of decomposition. Then DCT was applied to every sub-bands. After that, SVD was applied to the 12 sub-bands obtained by $5^{\text {th }}, 6^{\text {th }}$ and $7^{\text {th }}$ level decompositions. Like the host image, 2 level DWT was applied to the mark images by decomposing the HH sub-band. After DWT, DCT was applied to every sub-band. SVD was applied to the sub-bands obtained by the 2nd level of decomposition. The singular values of those sub-bands of the mark images were combined with the singular values of the sub-bands of the host image obtained by $5^{\text {th }}, 6^{\text {th }}$ and $7^{\text {th }}$ level of decompositions. In our technique, we used three separate images as watermarks. But, for comparison we redid our technique using the same image three times. We compared our work with [18] considering the attacks against which the robustness of the work [18] was tested. Figures 1.54-1.66 represent the comparison between the two techniques for different attacks. The authors of [18] tested the robustness of their developed technique against some attacks which could not be used to test the robustness of our proposed technique as there are some differences in the embedding algorithm between our proposed technique and the work [18]. But most of the attacks were used to test the robustness of our proposed technique.

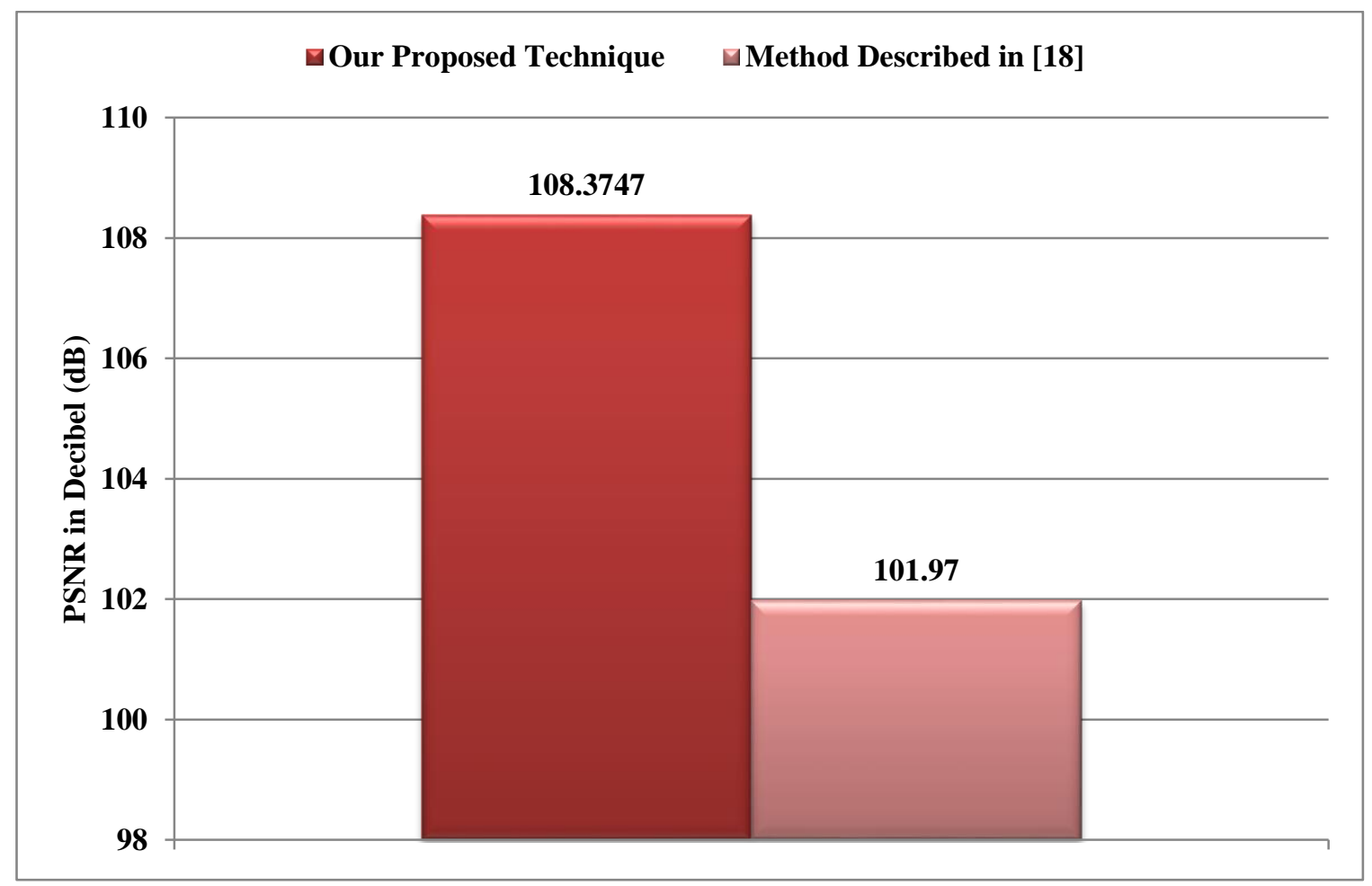

Fig. 1.54: Comparison between the Two Techniques in Terms of PSNR. 


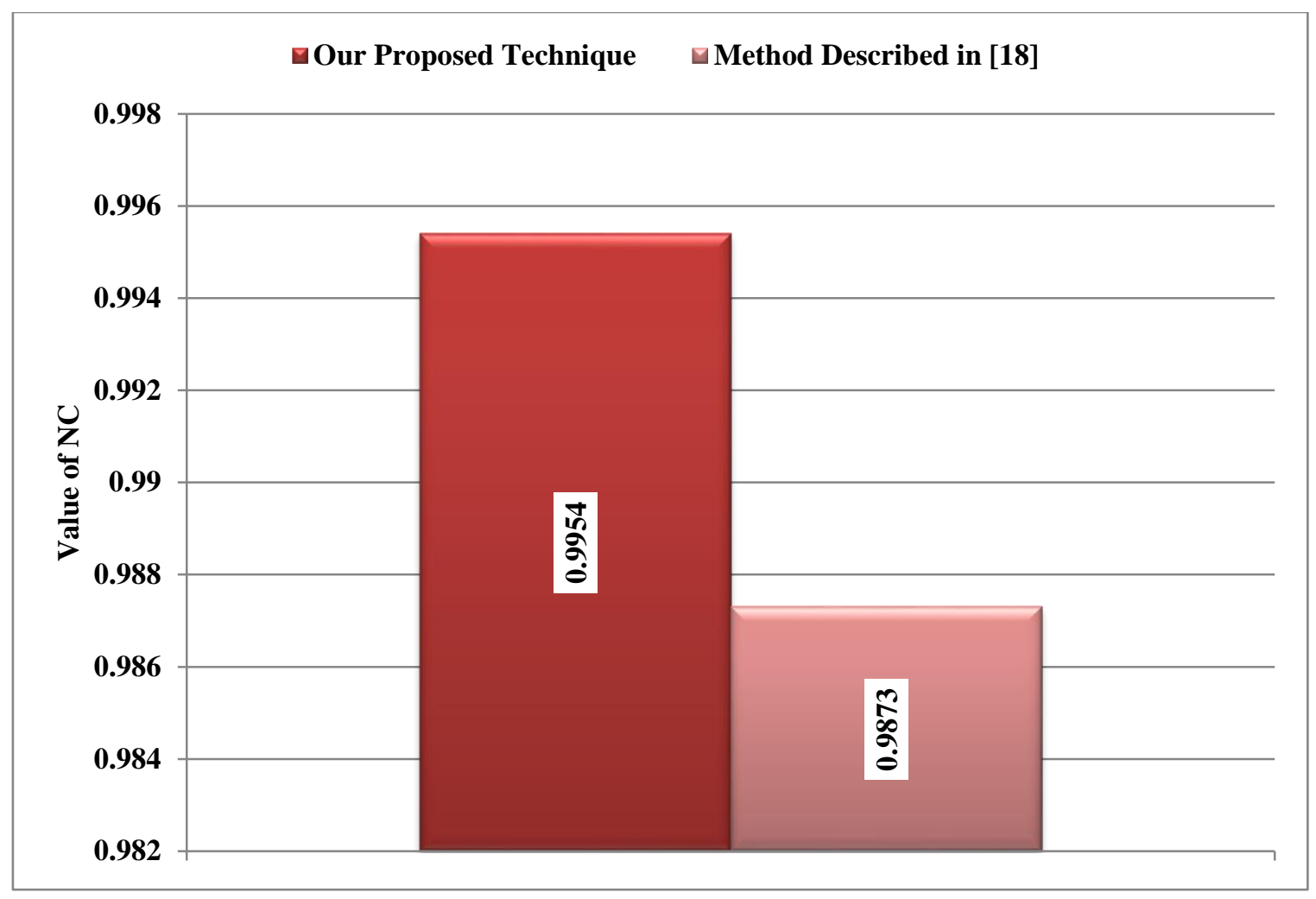

Fig. 1.55: Comparison between the Two Techniques in Terms of NC.

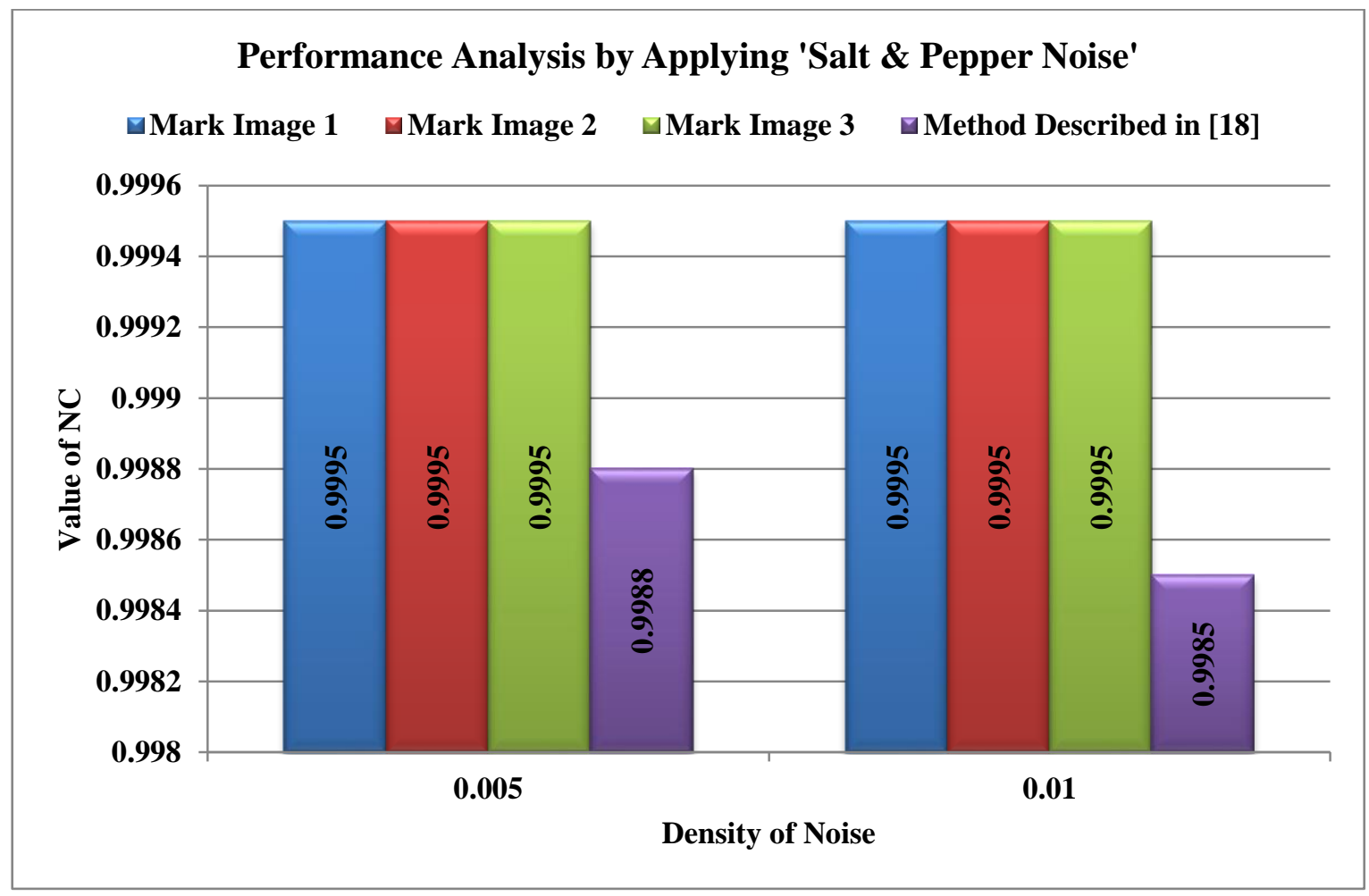

Fig. 1.56: Performance Analysis by Changing the Density of 'Salt \& Pepper Noise'. 


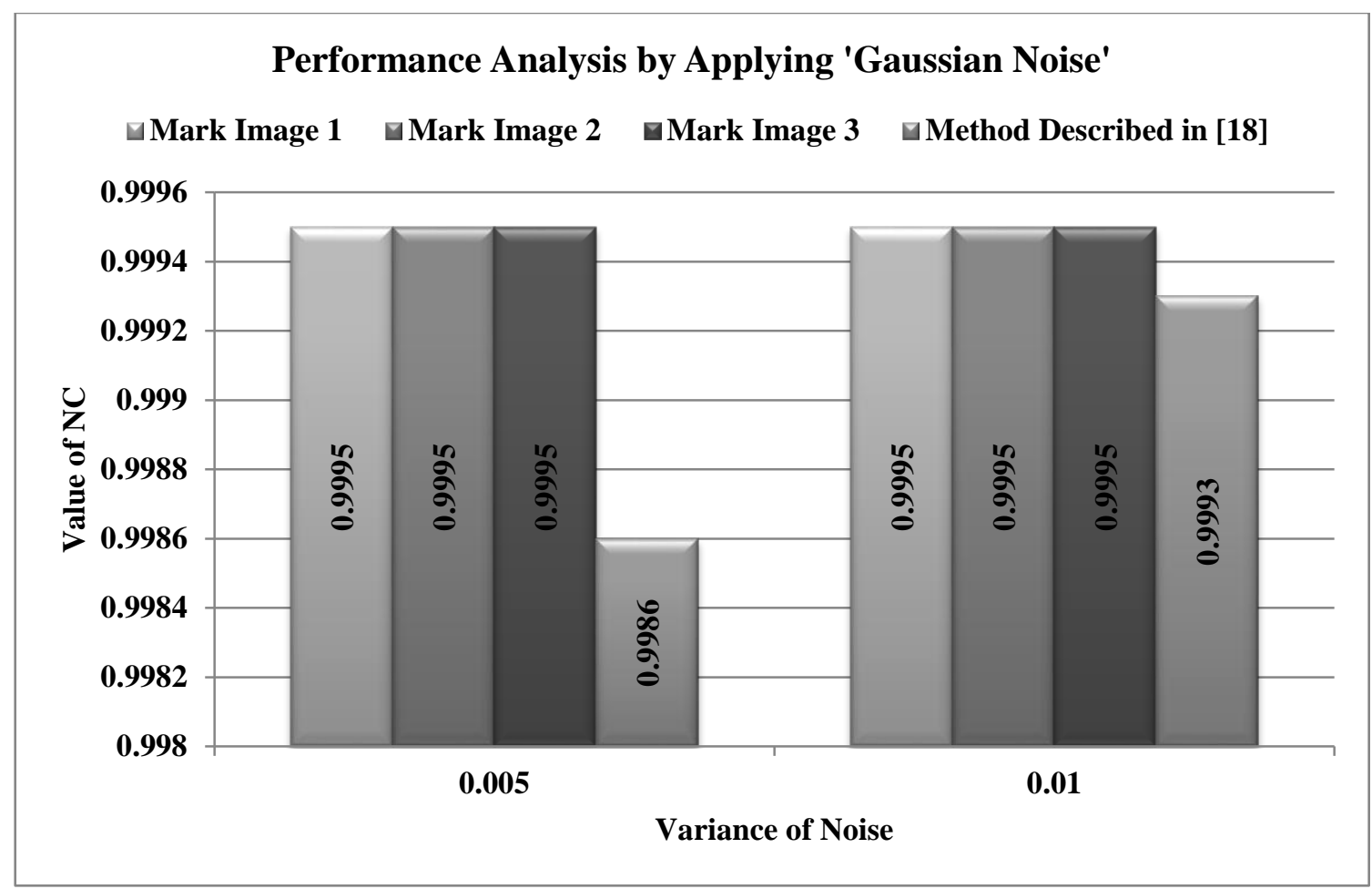

Fig. 1.57: Performance Analysis by Changing the Variance of 'Gaussian Noise'.

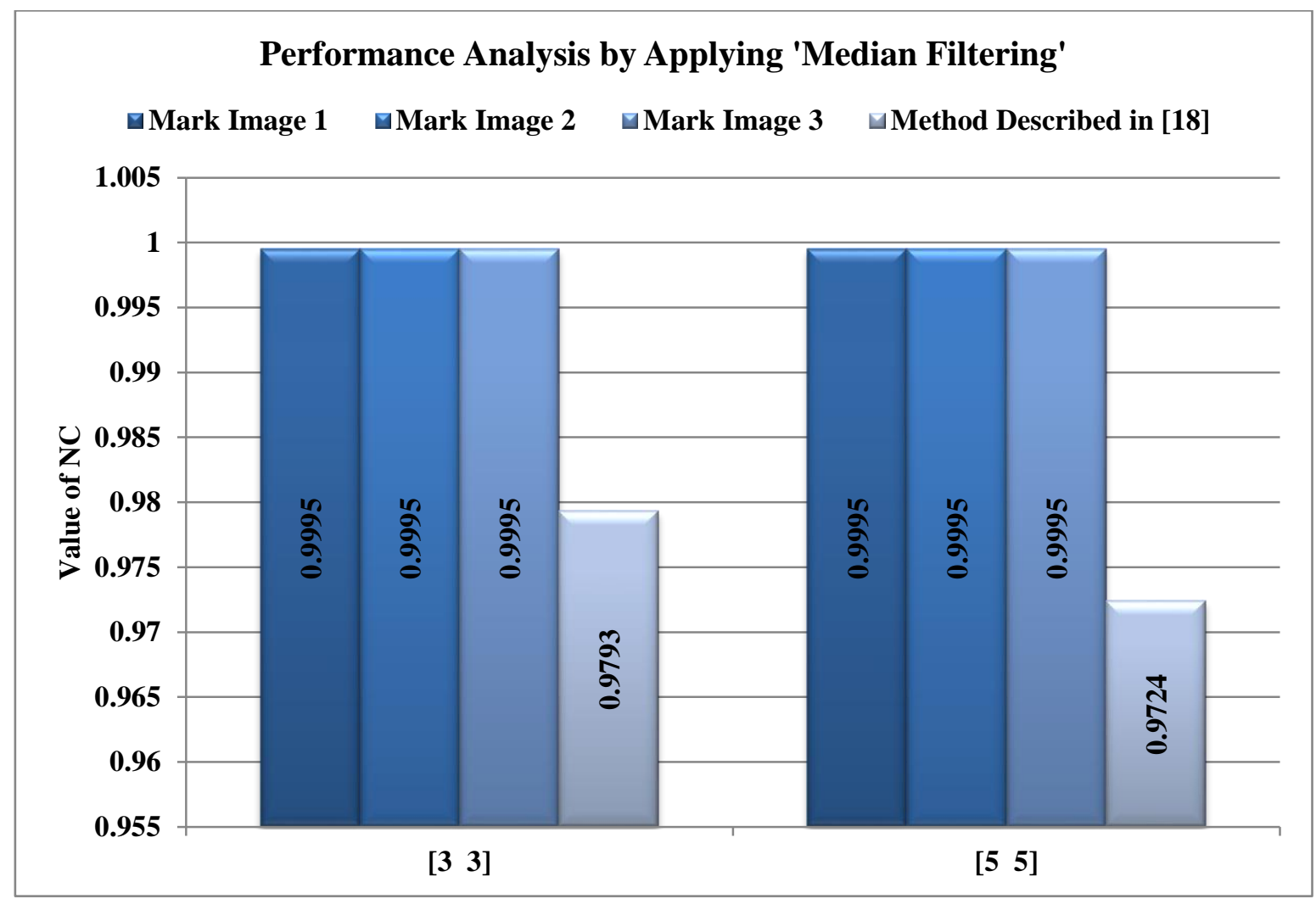

Fig. 1.58: Performance Analysis by Changing the Median of 'Median Filtering'. 


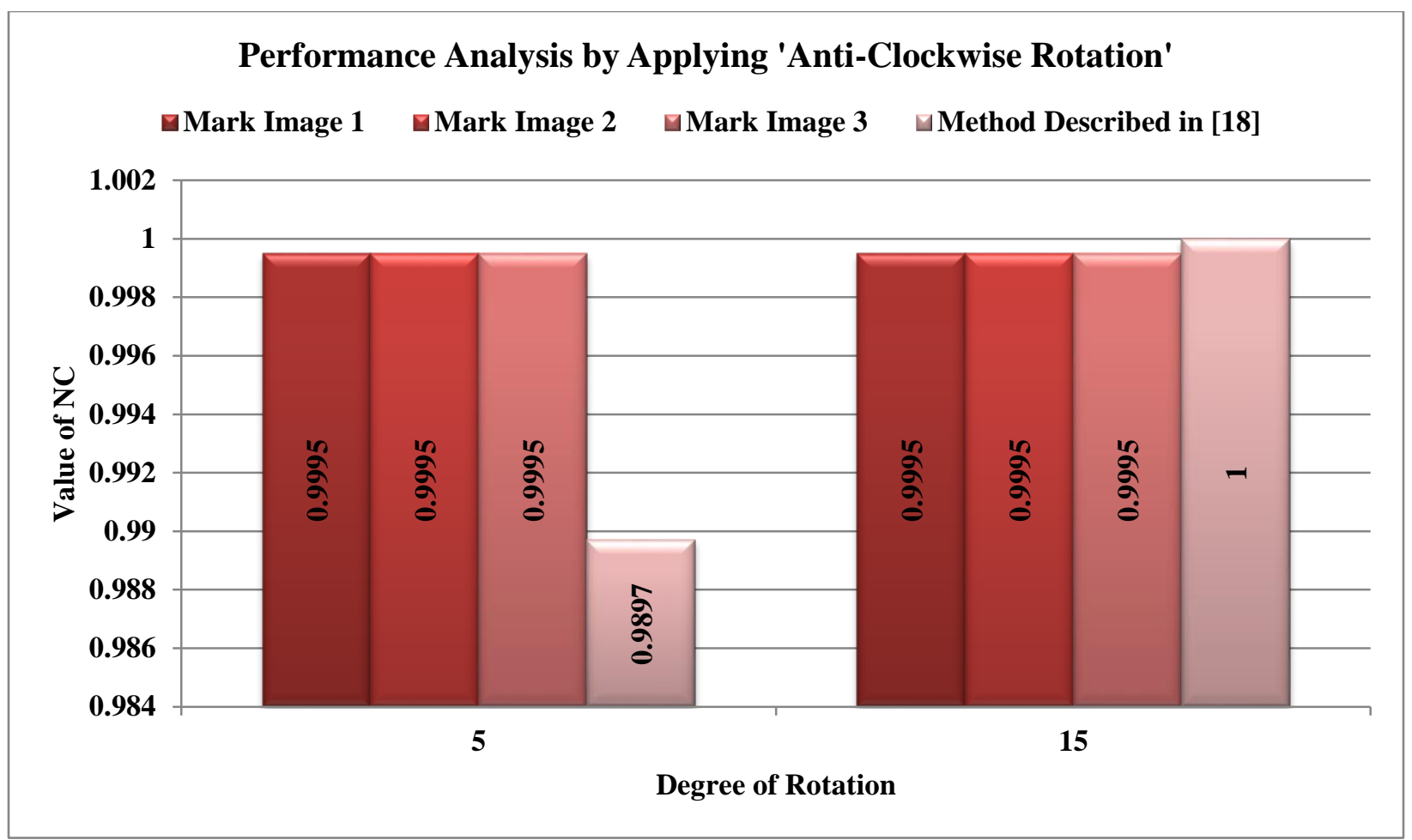

Fig. 1.59: Performance Analysis by Changing the Degree of Rotation in Anti-Clockwise Direction.

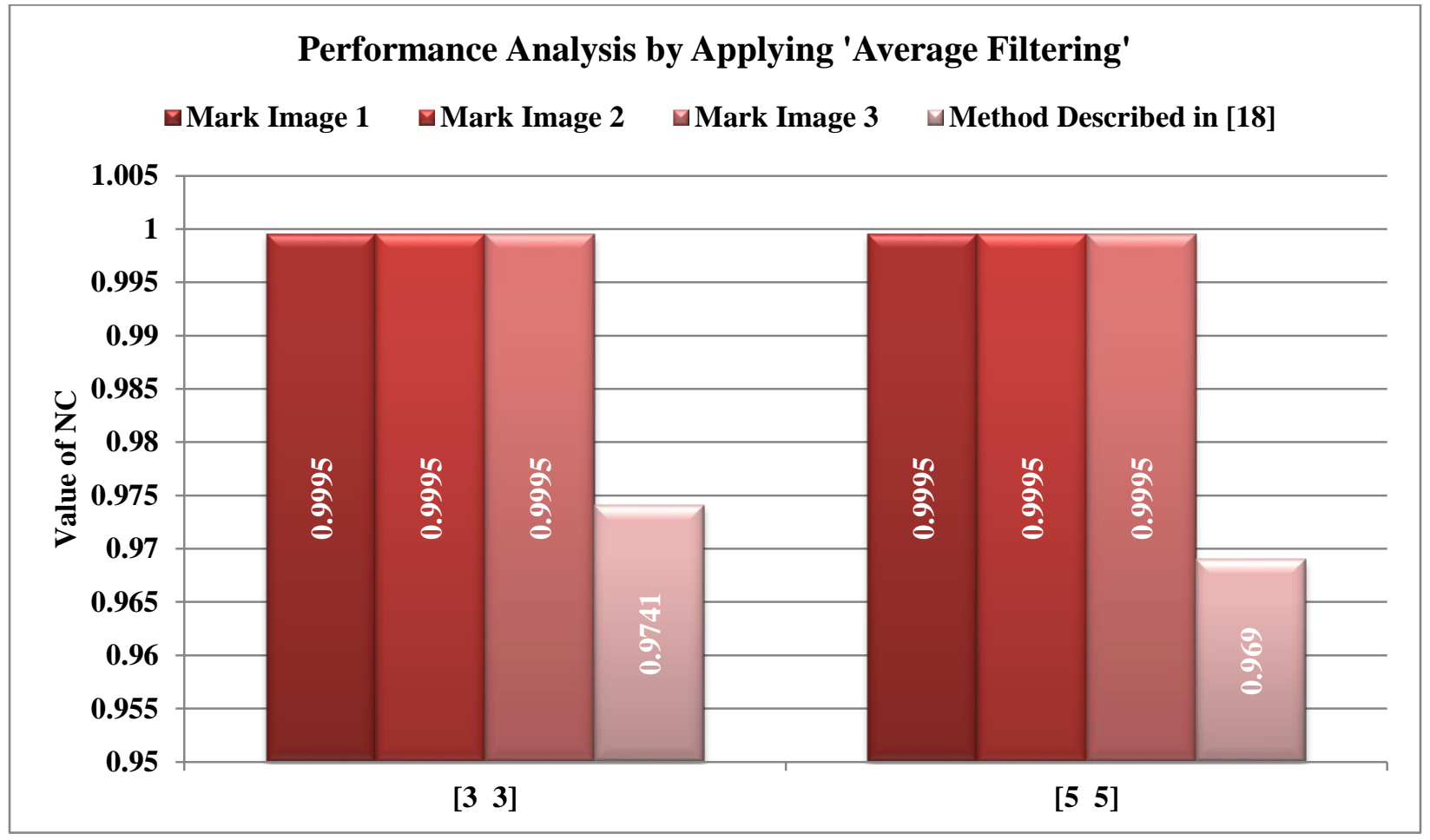

Fig. 1.60: Performance Analysis by Applying 'Average Filtering'. 


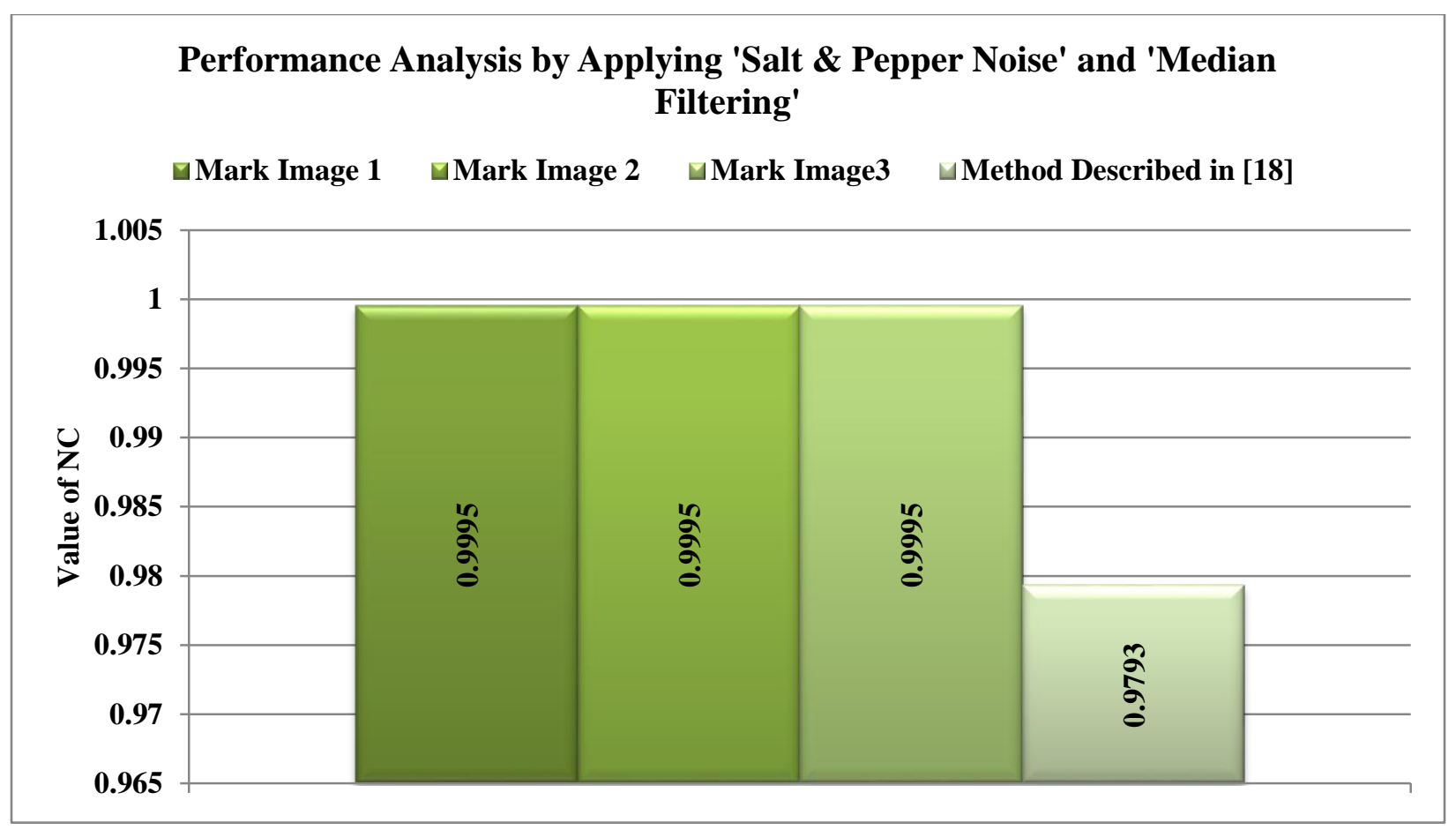

Fig. 1.61: Performance Analysis by Applying Hybrid Attacks ('Salt \& Pepper Noise' and 'Median Filtering').

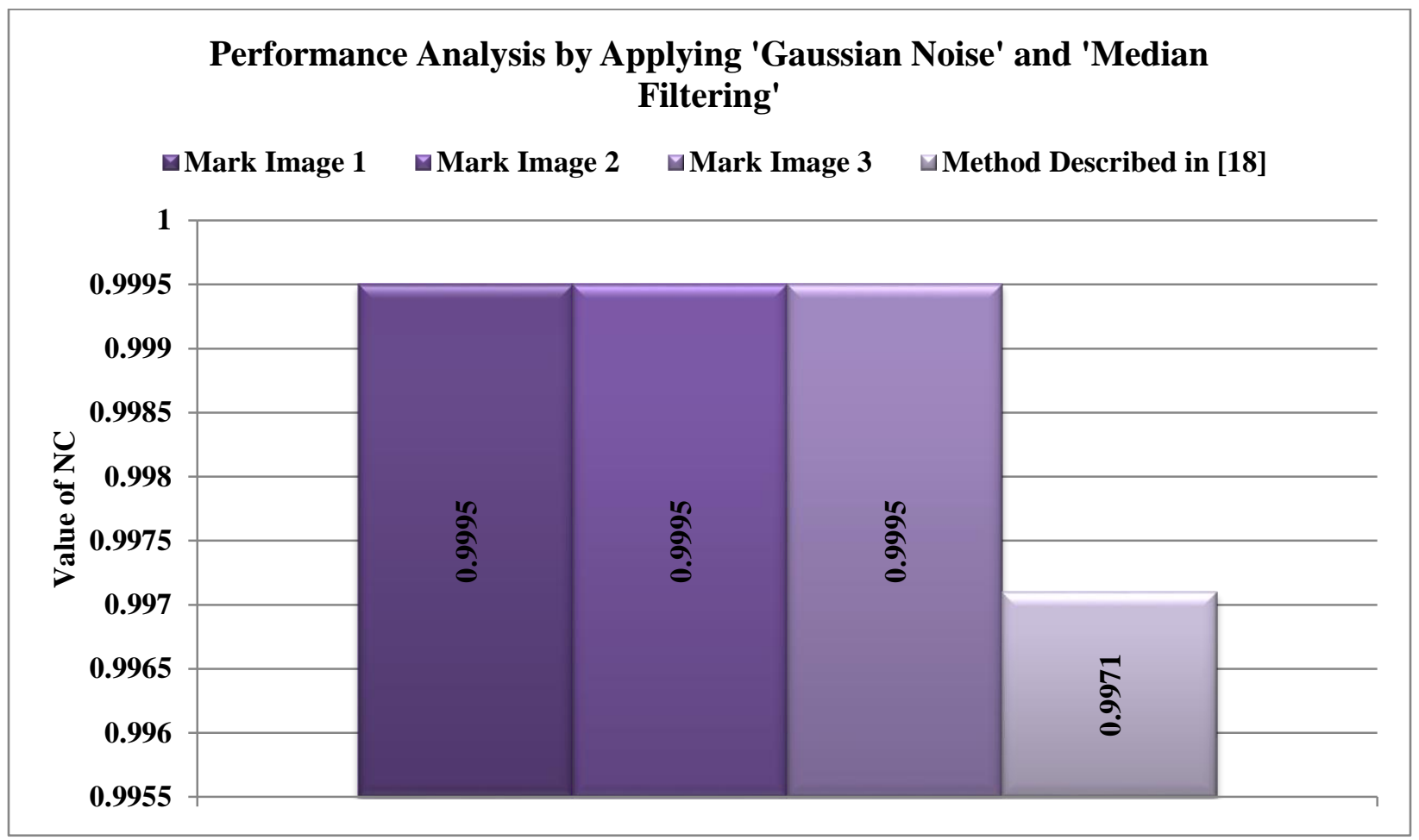

Fig. 1.62: Performance Analysis by Applying Hybrid Attacks ('Gaussian Noise' and 'Median Filtering'). 


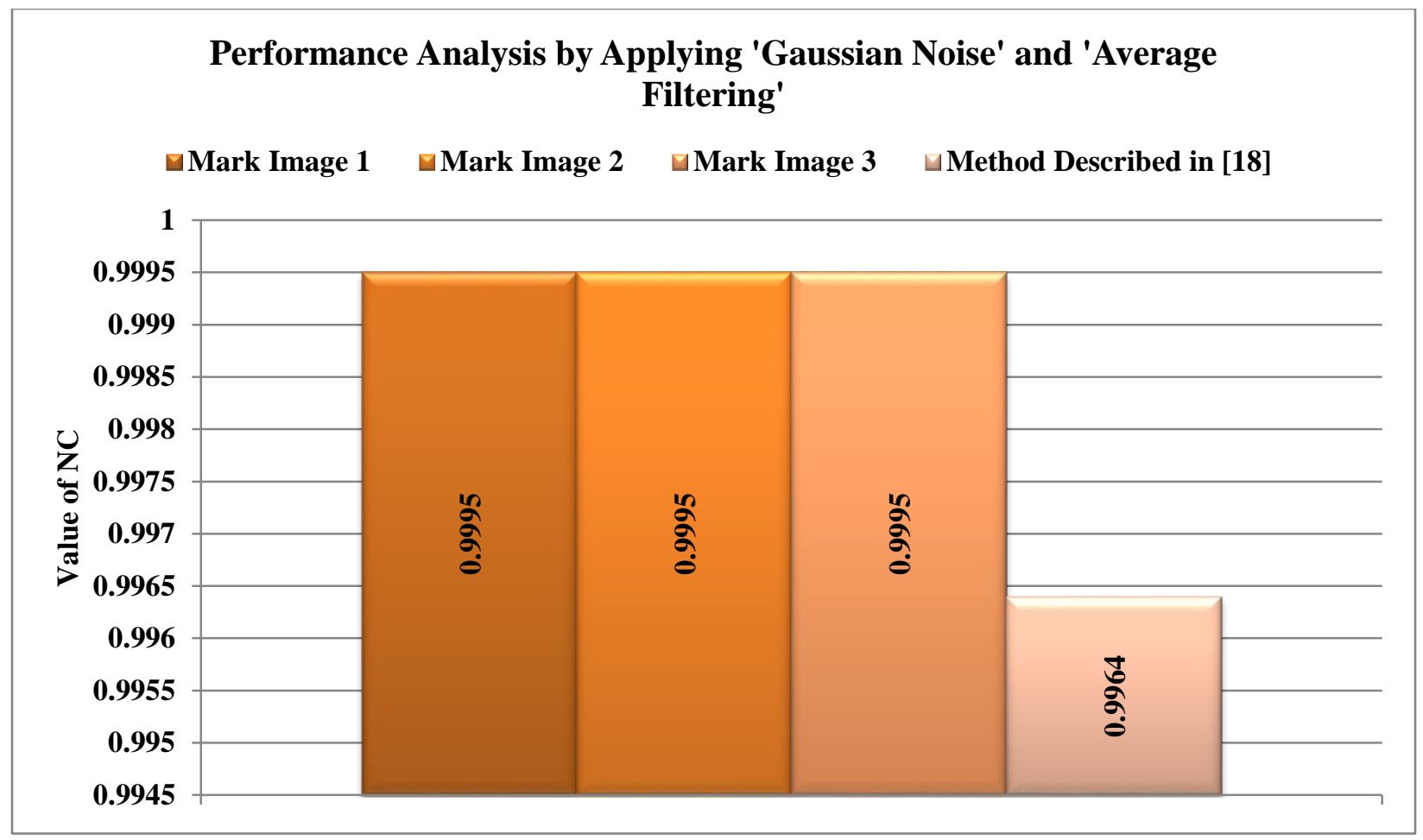

Fig. 1.63: Performance Analysis by Applying Hybrid Attacks ('Gaussian Noise' and 'Average Filtering').

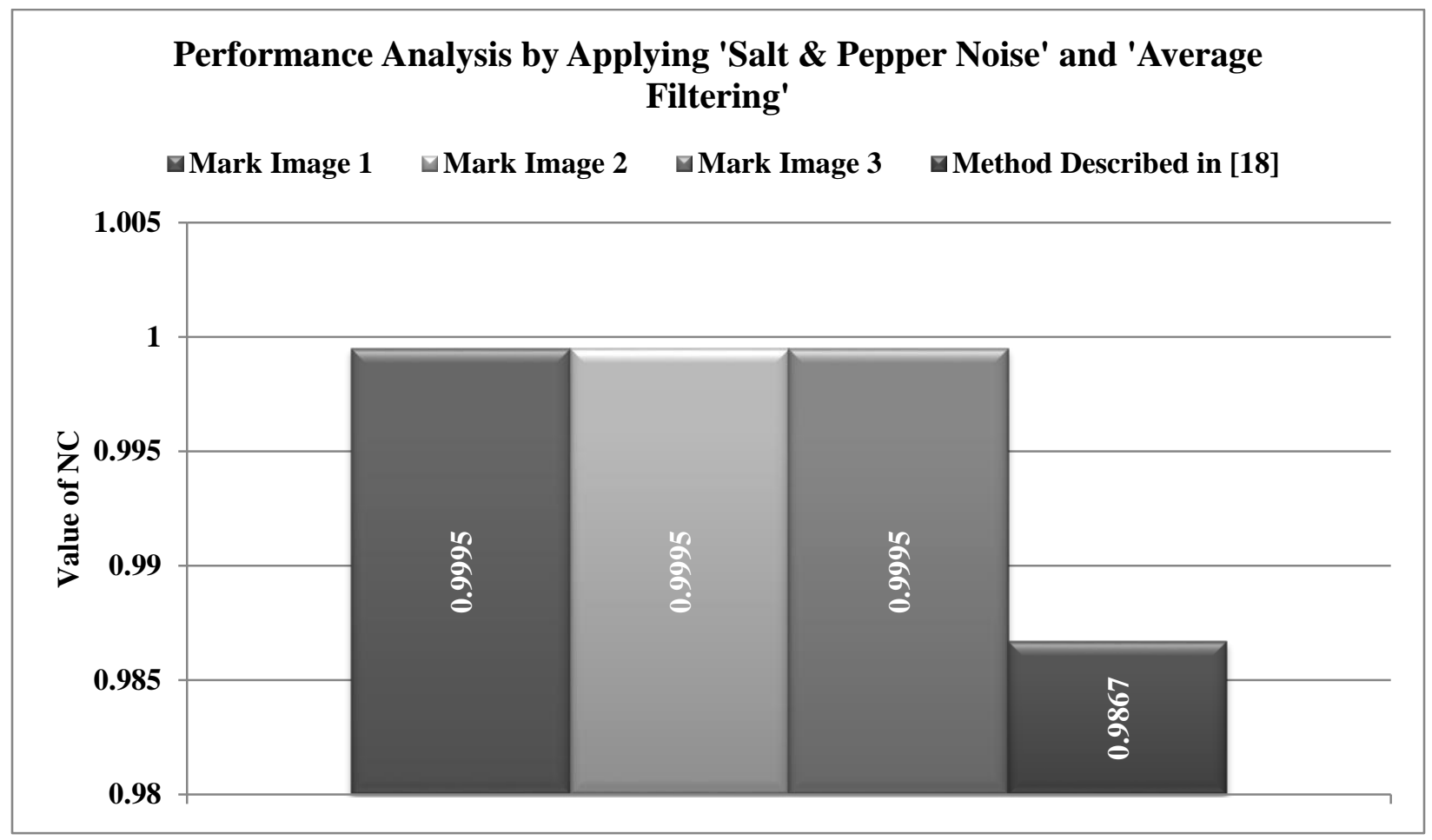

Fig. 1.64: Performance Analysis by Applying Hybrid Attacks ('Salt \& Pepper Noise' and 'Average Filtering'). 


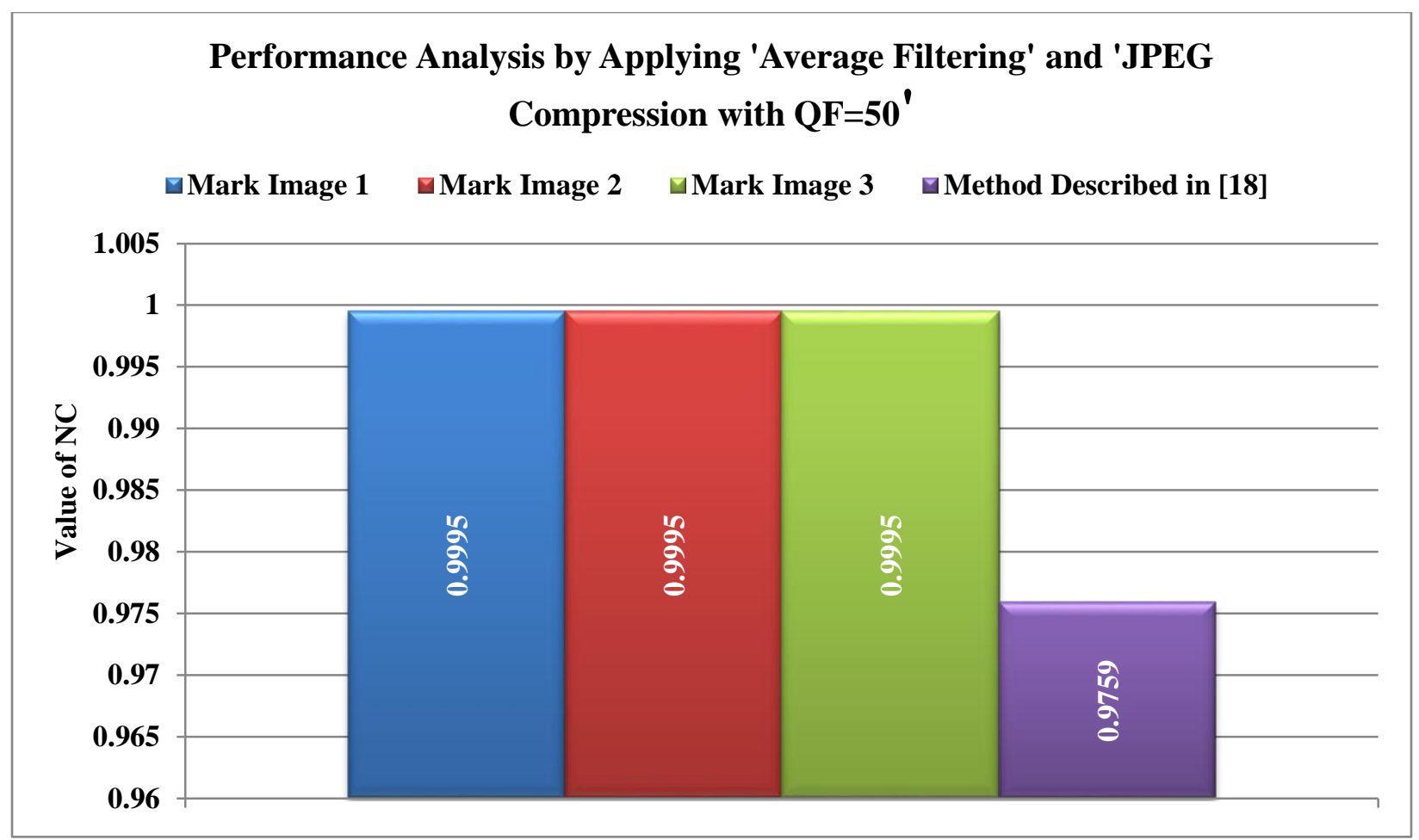

Fig. 1.65: Performance Analysis by Applying Hybrid Attacks ('Average Filtering' and 'JPEG Compression with $\mathrm{QF}=50$ ').

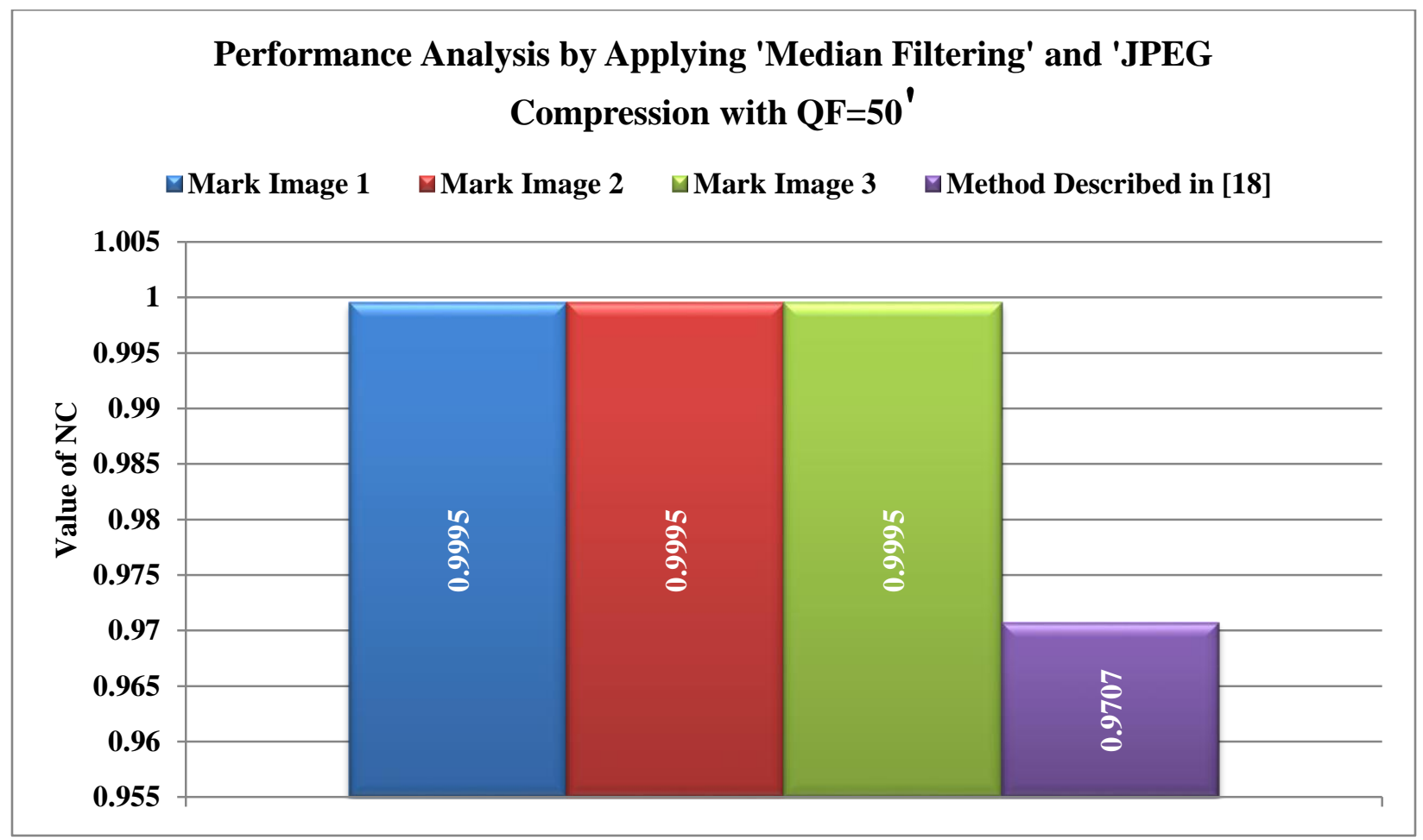

Fig. 1.66: Performance Analysis by Applying Hybrid Attacks ('Median Filtering' and 'JPEG Compression with $\mathrm{QF}=50$ '). 
After comparison with [18], it can be said that our proposed technique performed better than the work developed in [18] as we achieved higher PSNR value as well as NC after applying several attacks.

\section{References:}

[1] Afroja Akter,, Nur-E-Tajnina and Mohammad Ahsan Ullah, "Digital Image Watermarking Based on DWT-DCT: Evaluate for a New Embedding Algorithm",3rd International Conference on Informatics, electronics \& Vision 2014.

[2] V.Santhi and P,Arulmozhivarman, "Hadamard transform based adaptive visible/invisible watermarking scheme for digital images", Journal of information security and applications 18(2013), Elsevier, (http://dx.doi.org/10.1016/j.istr.2013.01.001), pp: 167-179.

[3] Fridrich, J., Goljan and M., Baldoza, A., "New Fragile Watermarking for Images".

[4] Ling-Yuan Hsu and Hwai-Tsu Hu, "Blind image watermarking via exploitation of inter-block prediction and visibility threshold in DCT domain", Elsevier J. Vis. Commun. Image R., 32 (2015), pp. 130-143.

[5] Jagdish Prasad Maheshwari, Mahendra Kumar, Garima Mathur, R.P. Yadavand Rajesh Kumar Kakerda, "Robust Digital Image Watermarking using DCT based Pyramid Transform via image compression", IEEE ICCSP (2015), pp. 1059-1063.

[6] Madhuri Rajawat and D.S Tomar, "A Secure Watermarking and Tampering detection technique on RGB Image using 2 Level DWT", Fifth International Conference on Communication Systems and Network Technologies, 2015 IEEE, pp:638-342.

[7] Anurag Mishra, Charu Agarwal, Arpita Sharma, and Punam Bedi, "Optimized gray-scale image watermarking using DWT-SVD and Firefly Algorithm", Elsevier Expert Systems with Applications, 41 (2014), pp. 7858-7867. 
[8] Pan-PanZheng, JunFeng, Zhan Li, and Ming-quan Zhou, "A Novel SVD and LS-SVM combination algorithm for blind watermarking" 2014 Elsevier Neurocomputing, 142 (2014), pp. 520-528.

[9] Shao-li Jia, "A novel blind color images watermarking based on SVD", Optik, 125 (2014), pp. 2868-2874.

[10] Nasrin M. Makbol and Bee Ee Khoo, "A new robust and secure digital image watermarking scheme based on the integer wavelet transform and singular value decomposition", 2014 Published by Elsevier Inc., http://dx.doi.org/10.1016/j.dsp.2014.06.0121051-2004.

[11] Palak Jaina and Umesh Ghanekarb, " Robust watermarking technique for textured images", 6th International Conference on Smart Computing and Communications, ICSCC 2017, 7-8 December 2017, Kurukshetra, India.

[12] Andalibi Mehran and M. Damon Chandler, senior member IEEE, "Digital Image Watermarking via Adaptive Logo Texturization" IEEE Transactions on Image Processing, 24 (12) (December 2015).

[13] Mehto, A., \& Mehra, N. (2016). "Adaptive Lossless Medical Image Watermarking Algorithm Based on DCT \& DWT". Physics Procedia, 78, 88-94. https://doi.org/10.1016/j.procs.2016.02.015.

[14] Chowdhury, A., Zaman, H., \& Khan, N. N. (2014). Color Image Watermarking Technique by Featuring Joint DWT-DCT Domain in YIQ Color Space, 4, 1-6.

[15] C.S. Woo, J. Du, and B. Pham, "Multiple watermarking methods for privacy control and tamper detection in medical images",In Proceedings of the Lovell, Brian C. \&Maeder, Anthony J. (Eds.) APRS Workshop on Digital Image Computing; Feb 21, Brisbane (South Bank), Queensland University of Technology Australia: (2005), pp. 59-64.

[16] A. Giakoumaki, S. Pavlopulos and D. Koutouris, "Multiple image watermarking applied to health information management". 
[17] Koushik Pal, G.Ghosh and M. Bhattacharya, " Biomedical Image Watermarking in Wavelet Domain for Data Integrity Using Bit Majority Algorithm and Multiple Copies of Hidden Information", American Journal of Biomedical Engineering 2012, 2(2): 29-37 DOI: 10.5923/j.ajbe.20120202.06.

[18] Zhou, X., Zhang, H., \& Wang, C. (2018)."A Robust Image Watermarking Technique Based on DWT, APDCBT, and SVD". SS symmetry. https://doi.org/10.3390/sym10030077 